\title{
The full basis theorem does not imply analytic wellordering
}

\author{
Vladimir Kanovei ${ }^{* \dagger} \quad$ Vassily Lyubetsky ${ }^{\ddagger}$
}

March 18, 2019

\begin{abstract}
We make use of a finite support product of $\omega_{1}$ clones of the Jensen minimal $\Pi_{2}^{1}$ singleton forcing to define a model in which every non-empty lightface analytically definable set of reals contains a lightface analytically definable real (the full basis theorem), but there is no lightface analytically definable wellordering of the continuum.
\end{abstract}

\footnotetext{
${ }^{*}$ IITP RAS and MIIT, Moscow, Russia, kanovei@googlemail.com - contact author.

${ }^{\dagger}$ Thankful the Department of Philosophy, Linguistics and Theory of Science at the University of Gothenburg and the Erwin Schrodinger International Institute for Mathematics and Physics (ESI) at Vienna for their hospitality and support in resp. May 2015 and December 2016.

${ }^{\ddagger}$ IITP RAS, Moscow, Russia, lyubetsk@iitp.ru.

${ }^{\S}$ Supported in part by RNF Grant \#14-50-00150.
} 
Introduction . . . . . . . . . . . . . . . . . . . . . . . . 3

Comments . . . . . . . . . . . . . . . . . . . . . . . . . . . 4

The structure of the paper . . . . . . . . . . . . . . . . . . . . . . . 5

I Basic constructions 6

4 Perfect trees .................................6 6

Perfect tree forcing notions . . . . . . . . . . . . . . . . . . . . . . . . . . . 7

Splitting construction .............................8

Multiforcings and multitrees . . . . . . . . . . . . . . . . . . . . . . . 9

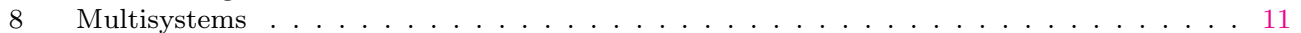

II Refinements

9 Refining perfect tree forcings . . . . . . . . . . . . . . . . . . . . . . 13

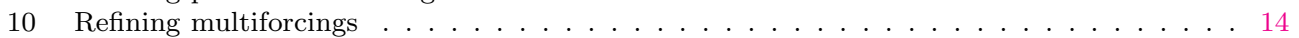

11 Generic refinement of a multiforcing . . . . . . . . . . . . . . . . . . . . . . 16

12 Preservation of density . . . . . . . . . . . . . . . . . . . . . . 19

III Structure of real names

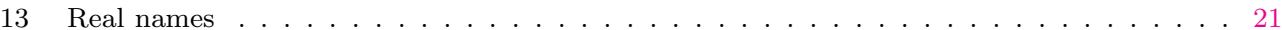

14 Direct forcing . . . . . . . . . . . . . . . . . . . . . . . . . . . . . 22

15 Locking real names . . . . . . . . . . . . . . . . . . . . . . . . . . . 23

16 Non-principal names and avoiding refinements . . . . . . . . . . . . . . . . . . . . . . . . 23

17 Generic refinements avoid non-principal names . . . . . . . . . . . . . . . . . . . . . . . 24

18 Consequences for reals in generic extensions . . . . . . . . . . . . . . . . . . . . . . . . . 26

19 Combining refinement types . . . . . . . . . . . . . . . . . . . . . . . . . 28

IV The forcing notion 29

20 Increasing sequences of small multiforcings . . . . . . . . . . . . . . . . . . . . . . . . . . . 29

21 Layer restrictions of multiforcings and deciding sets . . . . . . . . . . . . . . . . . . . . 30

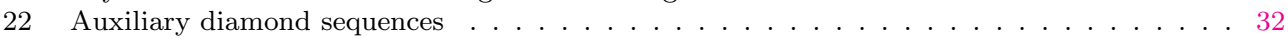

23 Key sequence theorem . . . . . . . . . . . . . . . . . . . . . . . . 33

24 Key product forcing . . . . . . . . . . . . . . . . . . . . . . . . . . 36

$\mathrm{V}$ Auxiliary forcing relation 38

25 Auxiliary forcing: preliminaries . . . . . . . . . . . . . . . . . . . . 38

26 Auxiliary forcing . . . . . . . . . . . . . . . . . . . . . . . . . . . . . . . . . . . . 39

27 Forcing simple formulas . . . . . . . . . . . . . . . . . . . . . . . . . . 40

28 Tail invariance . . . . . . . . . . . . . . . . . . . . . . . . . . . . . . . . . 42

29 Permutations . . . . . . . . . . . . . . . . . . . . . . . . . . . . 43

30 Forcing with subsequences of the key multisequence . . . . . . . . . . . . . . . . . . 45

VI The model

31 Key generic extension and subextensions . . . . . . . . . . . . . . . . . . . . . . . . 48

32 Definability of generic reals . . . . . . . . . . . . . . . . . . . . . . . . . . . . . . . . . 49

33 Elementary equivalence ..........................51

34 Non-wellorderability . . . . . . . . . . . . . . . . . . . . . . . . . . . . . . . . . . 53

35 Proof of the main theorem . . . . . . . . . . . . . . . . . . . . . 55

References

\begin{tabular}{ll} 
Index & 58 \\
\hline
\end{tabular} 


\section{Introduction}

The uniformization problem, introduced by Luzin [22, 23], as well as the related basis problem, are well known in modern set theory. (See Moschovakis [24], Kechris [21], Hauser and Schindler [7] for both older and more recent studies.) In particular, it is known that every non-empty $\Sigma_{2}^{1}$ set of reals contains a $\Delta_{2}^{1}$ real, but on the other hand, it is consistent that there exists a non-empty $\Pi_{2}^{1}$ set of reals containing even no ordinal-definable real.

The negative part of this result was strengthened in [19] to the effect that the counter-example set $X \subseteq \omega^{\omega}$ is a $\Pi_{2}^{1} \mathrm{E}_{0}$-equivalence class (hence, a countable set), see related discussions at the Mathoverflow exchange desk ${ }^{1}$ and at $\mathrm{FOM}^{2}$. Recall that $\mathrm{E}_{0}$ is an equivalence relation on $\omega^{\omega}$ defined so that $x \mathrm{E}_{0} y$ iff $x(n)=$ $y(n)$ for all but finite $n$.

As for the positive direction, the most transparent way to get a basis result is to make use of an analytically definable wellordering $<$ of the reals, which enables one to pick the <-least real in each non-empty set of reals. This leads to the question: is the existence of an analytically definable wellordering $<$ of the reals independent of the basis theorem. We answer it in the positive:

Theorem 1.1. In a suitable generic extension of $\mathbf{L}$, it is true that in which every non-empty lightface analytically definable set of reals contains a lightface analytically definable real (the full basis theorem), but there is no lightface analytically definable wellordering of the continuum.

More precisely, there is a cardinal-preserving generic extension $\mathbf{L}[X]$ of $\mathbf{L}$, such that $X=\left\langle x_{\xi k}\right\rangle_{\xi<\omega_{1}^{\mathrm{L}} \wedge k<\omega}$, where each $x_{\xi k}$ is a real in $2^{\omega}$, and in addition

(I) if $m<\omega$ then the submodel $\mathbf{L}\left[X_{m}\right]$ admits a $\Delta_{m+3}^{1}$ wellordering of the reals of length $\omega_{1}$, where $X_{m}=\left\langle x_{\xi k}\right\rangle_{\xi<\omega_{1}^{\mathbf{L}} \wedge k<m}$;

(II) if $m<\omega$ then $\omega^{\omega} \cap \mathbf{L}\left[X_{m}\right]$ is a $\Sigma_{m+3}^{1}$ set in $\mathbf{L}[x]$;

(III) if $m<\omega$ then $\mathbf{L}\left[X_{m}\right]$ is an elementary submodel of $\mathbf{L}[x]$ w.r.t. all $\Sigma_{m+2}^{1}$ formulas with reals in $\mathbf{L}\left[X_{m}\right]$ as parameters;

(IV) it is true in $\mathbf{L}[X]$ that there is no lightface analytically definable wellordering of the reals.

To see that the additional claims imply the main claim (the full basis theorem), let, in $\mathbf{L}[X], Z \subseteq \omega^{\omega}$ be a non-empty $\Sigma_{m+2}^{1}$ set of reals. Then $Z^{\prime}=$ $Z \cap \mathbf{L}\left[X_{m}\right]$ is a $\Sigma_{m+3}^{1}$ set by (II), and $Z^{\prime} \neq \varnothing$ by (III). It remains to pick the least real in $Z^{\prime}$ in the sense of the lightface $\Delta_{m+3}^{1}$ wellordering given by (I).

\footnotetext{
1 A question about ordinal definable real numbers. Mathoverflow, March 09, 2010. http://mathoverflow.net/questions/17608.

${ }_{2}$ Ali Enayat. Ordinal definable numbers. FOM Jul 23, 2010. http://cs.nyu.edu/pipermail/fom/2010-July/014944.html
} 


\section{Comments}

To prove the theorem, we define, in $\mathbf{L}$, a system of forcing notions $\mathbb{P}_{\xi k}, \xi<\omega_{1}$ and $k<\omega$, whose finite-support product $\mathbb{P}=\prod_{\xi, k} \mathbb{P}_{\xi k}$ adds an array $X=$ $\left\langle x_{\xi k}\right\rangle_{\xi<\omega_{1}, k<\omega}$ of reals $x_{\xi k}$ to $\mathbf{L}$, such that (I), (II), (III), (IV) hold in $\mathbf{L}[X]$.

Regarding the history of this research, in goes down to Jensen [10], where a forcing $\mathbb{J}=\bigcup_{\alpha<\omega_{1}} \mathbb{J}_{\alpha}$ is defined in $\mathbf{L}$, the constructible universe, such that each $\mathbb{J}_{\alpha}$ is a countable set of perfect trees in $2^{<\omega}$, the canonical $\mathbb{J}$-generic real is a single $\mathbb{J}$-generic real in the extension, and 'being a $\mathbb{J}$-generic real' is a $\Pi_{2}^{1}$

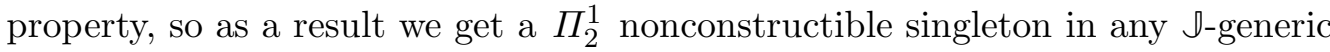
extension of $\mathbf{L}$. See 28A in [8] for a more modern exposition of Jensen's forcing.

A nonconstructible $\Pi_{2}^{1}$ singleton also was defined in [9] by means of the almost-disjoint forcing, yet the construction in [10] has the advantage of minimal$i t y$ of $\mathbb{J}$-generic reals and some other advantages (as well as some disadvantages).

Jensen's forcing construction (including its iterations) was exploited by Abraham $[1,2]$, including a definable minimal collapsing real. Another modification of Jensen's forcing construction in [11] yields such a forcing notion in $\mathbf{L}$ that any extension of $\mathbf{L}$, containing two generic reals $x \neq y$, necessarily satisfies $\omega_{1}^{\mathbf{L}}<\omega_{1}$. See $[3,15]$ on some other modifications in coding purposes.

A different modification of Jensen's forcing construction was engineered in [16] in order to define an extension of $\mathbf{L}$ in which, for a given $n \geq 2$, there is a nonconstructible $\Pi_{n}^{1}$ singleton while all $\Sigma_{2}^{1}$ reals are constructible. (An abstract appeared in [14].) The idea is to complicate the inductive construction of Jensen's sequence $\overrightarrow{\mathbb{J}}=\left\langle\mathbb{J}_{\alpha}\right\rangle_{\alpha<\omega_{1}}$ in $\mathbf{L}$ by the requirement that it intersects any set, of a certain definability level, dense in the collection of all possible countable initial steps of the construction. The same inner genericity idea, with respect to the Jensen - Johnsbraten forcing notion in [11], was developed in [18].

Such an inner genericity modification of the Jensen - Solovay almost-disjoint forcing [9] was developed in [6] towards some great results which unfortunately have never been published in a mathematical journal. Except for a one result, a model in which the set of all analytically definable reals is equal to the set of all constructible reals, independently obtained in [17]. We employ the inner definable genericity idea here in such a way that if $m<\omega$ then the $m$-tail $\left\langle\mathbb{P}_{\xi k}\right\rangle_{\xi<\omega_{1} \wedge k \geq m}$ of the forcing construction, bears an amount of inner definable genericity which strictly depends on $m$. (See Definition 21.1, where a key concept is introduced.)

Ali Enayat (Footnote 2) conjectured that some definability questions can be solved by finite-support products of Jensen's [10] forcing $\mathbb{J}$. Enayat demonstrated in [4] that a symmetric part of the $\mathbb{J}^{\omega}$-generic extension of $\mathbf{L}$ definitely yields a model of ZF (not a model of ZFC!) in which there is a Dedekind-finite infinite $\Pi_{2}^{1}$ set of reals with no OD elements. Following the conjecture, we proved in [12] that indeed it is true in a $\mathbb{J}^{\omega}$-generic extension of $\mathbf{L}$ that the set of $\mathbb{J}$-generic 
reals is a countable non-empty $\Pi_{2}^{1}$ set with no OD elements. We also proved in [13] that the existence of a $\Pi_{2}^{1} \mathrm{E}_{0}$-class with no OD elements is consistent with ZFC, using a $E_{0}$-invariant version of Jensen's forcing. We further employed another finite-support product of Jensen's forcing to define a generic extension of $\mathbf{L}$ where there is a $\Pi_{2}^{1}$ set $P \subseteq \omega^{\omega} \times \omega^{\omega}$ which has countable cross-sections $P_{x}=\{y:\langle x, y\rangle \in P\}$ and is non-uniformizable by any projective set [20].

Acknowledgement. The idea of making use of a suitable finite-support product of Jensen-like forcing notions in order to obtain a model, in which the full basis theorem holds but there is no lightface analytically definable wellordering of the continuum, was communicated to an author of this paper (VK) by Ali Enayat in 2015, and we thank Ali Enayat for fruitful discussions and helpful ideas.

\section{The structure of the paper}

The general organization of the paper is as follows. Chapter I contains a general formalism related to forcing by perfect trees and finite-support products, convenient for our goals. Following Jensen [10], we consider forcing notions of the form $\mathbb{P}=\bigcup_{\alpha<\lambda} \mathbb{P}_{\alpha}$, where $\lambda<\omega_{1}$ and each $\mathbb{P}_{\alpha}$ is a countable set of perfect trees in $2^{<\omega}$. Each term $\mathbb{P}_{\alpha}$ has to satisfy some routine conditions of refinement with respect to the previous terms, in particular, to make sure that each $\mathbb{P}_{\alpha}$ remains pre-dense at further steps. Also, each $\mathbb{P}_{\alpha}$ has to lock some dense sets in $\bigcup_{\xi<\alpha} \mathbb{P}_{\xi}$ so that they remain pre-dense at further steps as well. And this procedure has to be extended from single forcing notions to their finite-support products. These issues are dealt with in Chapter II.

Then we consider real names with respect to finite-support products of perfecttree forcing notions in Chapter III. Here the key issue is to make sure that if $\mathbb{P}$ is a factor in a product forcing considered then there is no other $\mathbb{P}$-generic real in the whole product extension except for the obvious one.

In Chapter IV we define the forcing notion $\mathbb{P}=\prod_{\xi<\omega_{1}, k<\omega} \mathbb{P}_{\xi k}$ to prove the main theorem, in the form of a limit of a certain increasing sequence of countable products of countable perfect-tree forcing notions. Quite a complicated construction of this sequence in $\mathbf{L}$ involves ideas related to diamond-style constructions, as well as to some sort of definable genericity, as explained above.

The forcing $\mathbb{P}$ is not analytically definable; basically, each $k$-th layer $\left\langle\mathbb{P}_{\xi k}\right\rangle_{\xi<\omega_{1}}$ belongs to $\Delta_{k+4}^{1}$. But it is a key property that the $\mathbb{P}$-forcing relation restricted to $\Sigma_{n}^{1}$ formulas is essentially $\Sigma_{n}^{1}$. We prove this in Chapter V, with the help of an auxiliary forcing notion forc. We also establish the invariance of forc with respect to countable-support permutations of $\omega_{1} \times \omega$.

We finally prove Theorem 1.1 in Chapter VI, on the base of the results obtained in two previous chapters. 


\section{Basic constructions}

We begin with some basic things: perfect trees in the Cantor space $2^{\omega}$, perfect tree forcing notions (those which consist of perfect trees), their finite-support products, and a splitting construction of perfect trees.

\section{Perfect trees}

Let $2^{<\omega}$ be the set of all strings (finite sequences) of numbers 0,1 . If $t \in 2^{<\omega}$ and $i=0,1$ then $t^{\wedge} k$ is the extension of $t$ by $k$. If $s, t \in 2^{<\omega}$ then $s \subseteq t$ means that $t$ extends $s$, while $s \subset t$ means proper extension. If $s \in 2^{<\omega}$ then $\operatorname{lh}(s)$ is the length of $s$, and $2^{n}=\left\{s \in 2^{<\omega}: \operatorname{lh}(s)=n\right\}$ (strings of length $n$ ).

A set $T \subseteq 2^{<\omega}$ is a tree iff for any strings $s \subset t$ in $2^{<\omega}$, if $t \in T$ then $s \in T$. Every non-empty tree $T \subseteq 2^{<\omega}$ contains the empty string $\Lambda$. If $T \subseteq 2^{<\omega}$ is a tree and $s \in T$ then put $T \uparrow_{s}=\{t \in T: s \subseteq t \vee t \subseteq s\}$.

Let PT be the set of all perfect trees $\varnothing \neq T \subseteq 2^{<\omega}$. Thus a non-empty tree $T \subseteq 2^{<\omega}$ belongs to PT iff it has no endpoints and no isolated branches. Then there is a largest string $s \in T$ such that $T=T \uparrow_{s}$; it is denoted by $s=\operatorname{stem}(T)$ (the stem of $T$ ); we have $s^{\wedge} 1 \in T$ and $s^{\frown} 0 \in T$ in this case.

Definition 4.1 (perfect sets). If $T \in \mathbf{P T}$ then $[T]=\left\{a \in 2^{\omega}: \forall n(a\lceil n \in T)\}\right.$ is the set of all paths through $T$, a perfect set in $2^{\omega}$. Conversely if $X \subseteq 2^{\omega}$ is a perfect set then $\operatorname{tree}(X)=\{a\lceil n: a \in X \wedge n<\omega\} \in \mathbf{P T}$ and $[\operatorname{tree}(X)]=X$.

Trees $T, S \in \mathbf{P T}$ are almost disjoint, a.d. for brevity, iff the intersection $S \cap T$ is finite; this is equivalent to just $[S] \cap[T]=\varnothing$.

The simple splitting of a tree $T \in \mathbf{P T}$ consists of smaller trees

$$
T(\rightarrow 0)=T \uparrow_{\operatorname{stem}(T) \frown 0} \quad \text { and } \quad T(\rightarrow 1)=T \uparrow_{\operatorname{stem}(T) \wedge 1}
$$

in PT, so that $[T(\rightarrow i)]=\{x \in[T]: x(h)=i\}$, where $h=\ln (\operatorname{stem}(T))$. We let

$$
T(\rightarrow u)=T(\rightarrow u(0))(\rightarrow u(1))(\rightarrow u(2)) \ldots(\rightarrow u(n-1))
$$

for each string $u \in 2^{<\omega}, \ln (u)=n$; and separately $T(\rightarrow \Lambda)=T$.

Lemma 4.2. Suppose that $T \in \mathbf{P T}$. Then:

(i) if $u \in 2^{<\omega}$ then there is a string $s \in 2^{<\omega}$ such that $T(\rightarrow u)=T \uparrow_{s}$;

(ii) if $s \in 2^{<\omega}$ then there is a string $u \in 2^{<\omega}$ such that $T \uparrow_{s}=T(\rightarrow u)$;

(iii) if $\varnothing \neq U \subseteq[T]$ is a (relatively) open subset of $[T]$, or at least $U$ has a nonempty interior in $[T]$, then there is a string $s \in T$ such that $T \uparrow_{s} \subseteq U$. 
If $T \in \mathbf{P T}$ and $a \in 2^{\omega}$ then the intersection $T(\rightarrow a)=\bigcap_{n<\omega} T(\rightarrow a\lceil n)=$ $\left\{\boldsymbol{h}_{T}(a)\right\}$ is a singleton, and the map $\boldsymbol{h}_{T}$ is a canonical homeomorphism from $2^{\omega}$ onto $[T]$. Accordingly if $S, T \in \mathbf{P T}$ then the map $\boldsymbol{h}_{S T}(x)=\boldsymbol{h}_{T}\left(\boldsymbol{h}_{S}{ }^{-1}(x)\right)$ is a canonical homeomorphism from $[S]$ onto $[T]$.

\section{$5 \quad$ Perfect tree forcing notions}

A perfect-tree forcing notion is any non-empty set $\mathbb{P} \subseteq \mathbf{P T}$ such that if $s \in T \in \mathbb{P}$ then $T \uparrow_{s} \in \mathbb{P}$, or equivalently, by Lemma 4.2 , if $u \in 2^{<\omega}$ then $T(\rightarrow u) \in \mathbb{P}$. Let PTF be the set of all such forcing notions $\mathbb{P} \subseteq \mathbf{P T}$.

Example 5.1. If $s \in 2^{<\omega}$ then the tree $[s]=\left\{t \in 2^{<\omega}: s \subseteq t \vee t \subseteq s\right\}$ belongs to PT. The set $\mathbb{P}_{\text {coh }}=\left\{[s]: s \in 2^{<\omega}\right\}$ of all such trees (the Cohen forcing) is a regular perfect-tree forcing notion.

Lemma 5.2. Let $\mathbb{P} \in \mathbf{P T F}$. If $T \in \mathbb{P}$ and a set $X \subseteq[T]$ is (relatively) open (resp., clopen) in $[T]$, then there is a countable (resp., finite) set $\mathscr{S}$ of pairwise a.d. trees $S \in \mathbb{P}$, satisfying $\bigcup_{S \in \mathscr{S}}[S]=X$.

Lemma 5.3. (i) If $s \in T \in \mathbb{P} \in \mathbf{P T F}$ then $T \uparrow_{s} \in \mathbb{P}$.

(ii) If $\mathbb{P}, \mathbb{P}^{\prime} \in \mathbf{P T F}, T \in \mathbb{P}, T^{\prime} \in \mathbb{P}^{\prime}$, then there are trees $S \in \mathbb{P}, S^{\prime} \in \mathbb{P}^{\prime}$ such that $S \subseteq T, S^{\prime} \subseteq T^{\prime}$, and $[S] \cap\left[S^{\prime}\right]=\varnothing$.

Proof. (i) use Lemma 4.2. (ii) If $T=T^{\prime}$ then let $S=T(\rightarrow 0), S^{\prime}=T(\rightarrow 1)$. If say $T \nsubseteq T^{\prime}$ then let $s \in T \backslash T^{\prime}, S=T \uparrow_{s}$, and simply $S^{\prime}=T^{\prime}$.

Definition 5.4. A set $\mathbb{A} \subseteq \mathbf{P T}$ is an antichain iff any trees $T \neq T^{\prime}$ in $\mathbb{A}$ are a.d., that is, $[T] \cap\left[T^{\prime}\right]=\varnothing$. A forcing notion $\mathbb{P} \in \mathbf{P T F}$ is:

small, if it is countable;

special, if there is an antichain $\mathbb{A} \subseteq \mathbb{P}$ such that $\mathbb{P}=\left\{T \uparrow_{s}: s \in T \in \mathbb{A}\right\}$ - note that $\mathbb{A}$ is unique if exists; we write $\mathbb{A}=\operatorname{base}(\mathbb{P})$ (the base of $\mathbb{P}$ );

regular, if for any $S, T \in \mathbb{P}$, the intersection $[S] \cap[T]$ is clopen in $[S]$ or clopen in $[T]$ (or clopen in both $[S]$ and $[T]$ ).

Lemma 5.5. Let $\mathbb{P} \in \mathbf{P T F}$. If $\mathbb{P}$ is special and $S, T \in \mathbb{P}$ are not a.d., then they are comparable: $S \subseteq T$ or $T \subseteq S$.

If $\mathbb{P}$ is special then $\mathbb{P}$ is regular. If $\mathbb{P}$ is regular, then

(i) if $S, T \in \mathbb{P}$ are not a.d., then they are compatible in $\mathbb{P}$, that is, there is a tree $R \in \mathbb{P}$ such that $R \subseteq S \cap T$.

(ii) if $S_{1}, \ldots, S_{k} \in \mathbb{P}$ then there is a finite set of pairwise a.d. trees $R_{1}, \ldots, R_{n} \in$ $\mathbb{P}$ such that $\left[S_{1}\right] \cap \ldots \cap\left[S_{k}\right]=\left[R_{1}\right] \cup \ldots \cup\left[R_{n}\right]$. 
(iii) if $\mathscr{S}_{1}, \ldots, \mathscr{S}_{k}$ are finite collections of trees in $\mathbb{P}$ then there is a finite set of trees $R_{1}, \ldots, R_{n} \in \mathbb{P}$ such that $\bigcup_{S \in \mathscr{S}_{1}}[S] \cap \ldots \cap \bigcup_{S \in \mathscr{S}_{k}}[S]=\left[R_{1}\right] \cup \ldots \cup\left[R_{n}\right]$, and for any $\mathscr{S}_{i}$ and $R_{j}$, there is $S \in \mathscr{S}_{i}$ such that $R_{j} \subseteq S$.

Proof. (iii) Apply (ii) to every set of the form $\left[S_{1}\right] \cap \ldots \cap\left[S_{k}\right]$, where $S_{i} \in \mathscr{S}_{i}$, $\forall i$, then gather all trees $R_{i}$ obtained in one finite set.

Remark 5.6. Any set $\mathbb{P} \in \mathbf{P T F}$ can be considered as a forcing notion (if $T \subseteq T^{\prime}$ then $T$ is a stronger condition); then $\mathbb{P}$ adds a real $x \in 2^{\omega}$.

Lemma 5.7. If a set $G \subseteq \mathbb{P}$ is generic over a ground set universe $\mathbf{V}$ (resp., over a transitive model, e.g. L) then

(i) the intersection $\bigcap_{T \in G}[T]$ contains a single real $x=x[G] \in 2^{\omega}$, and

(ii) this real $x$ is $\mathbb{P}$-generic, in the sense that if $D \subseteq \mathbb{P}$ is dense in $\mathbb{P}$ and belongs to $\mathbf{V}$ (resp., to the ground model) then $x \in \bigcup_{T \in D}[T]$.

As usual, a set $D \subseteq \mathbb{P}$ is:

- open in $\mathbb{P}$, if for any trees $T \subseteq S$ in $\mathbb{P}, T \in D \Longrightarrow S \in D$;

- dense in $\mathbb{P}$, if for any $T \in \mathbb{P}$ there is $S \in D, S \subseteq T$;

- pre-dense in $\mathbb{P}$, if the set $D^{\prime}=\{T \in \mathbb{P}: \exists S \in D(T \subseteq S)\}$ is dense in $\mathbb{P}$.

\section{Splitting construction}

We proceed with an important splitting/fusion construction of perfect trees by means of infinite splitting systems of such trees.

Definition 6.1. Let FSS be the set of all finite splitting systems, that is, systems of the form $\varphi=\left\langle T_{s}\right\rangle_{s \in 2 \leq n}$, where $n=\operatorname{hgt}(\varphi)<\omega$ (the height of $\varphi$ ), each value $T_{s}=T_{s}^{\varphi}=\varphi(s)$ is a tree in $\mathbf{P T}$, and

$(*)$ if $s \in 2^{<n}$ and $i=0,1$ (so $s^{\frown} i \in 2^{\leq n}$ ) then $T_{s \wedge i} \subseteq T_{s}(\rightarrow i)$ - it easily follows that $\left[T_{s \sim 0}\right] \cap\left[T_{s \wedge 1}\right]=\varnothing$.

We add the empty system $\boldsymbol{\Lambda}$ to $\mathbf{F S S}$, with $\operatorname{hgt}(\boldsymbol{\Lambda})=-1$.

A tree $T$ occurs in $\varphi \in$ FSS if $T=\varphi(s)$ for some $s \in 2^{\leq \operatorname{hgt}(\varphi)}$. If all trees occurring in $\varphi$ belong to some $\mathbb{P} \in \mathbf{P T F}$ then say that $\varphi$ is a finite splitting system over $\mathbb{P}$, symbolically $\varphi \in \mathbf{F S S}(\mathbb{P})$.

Let $\varphi, \psi$ be systems in FSS. Say that $\varphi$ extends $\psi$, symbolically $\psi \preccurlyeq \varphi$, if $n=\operatorname{hgt}(\psi) \leq \operatorname{hgt}(\varphi)$ and $\psi(s)=\varphi(s)$ for all $s \in 2^{\leq n}$, and properly extends, $\psi \prec \varphi$, if in fact $\operatorname{hgt}(\psi)<\operatorname{hgt}(\varphi)$ strictly.

Each system $\varphi \in \mathbf{F S S}(\mathbb{P})$ with $\operatorname{hgt}(\varphi)=0$ consists essentially of a single tree $T_{\Lambda}^{\varphi} \in \mathbb{P}$. The next lemma provides systems of arbitrary height. 
Lemma 6.2. Assume that $\mathbb{P} \in \mathbf{P T F}$. If $n \geq 1$ and $\psi=\left\langle T_{s}\right\rangle_{s \in 2 \leq n} \in \mathbf{F S S}(\mathbb{P})$ then there is a system $\varphi=\left\langle T_{s}\right\rangle_{s \in 2 \leq n+1} \in \mathbf{F S S}(\mathbb{P})$ which properly extends $\psi$.

Proof. If $s \in 2^{n}$ and $i=0,1$ then let $T_{s} \frown i=T_{s}(\rightarrow i)$.

The next well-known lemma belongs to the type of splitting/fusion lemmas widely used in connection with the perfect set forcing and some similar forcings.

Lemma 6.3. Let $\mathbb{P} \in \mathbf{P T F}$. Then there is an $\prec$-increasing sequence $\left\langle\varphi_{n}\right\rangle_{n<\omega}$ of systems in $\mathbf{F S S}(\mathbb{P})$. And if $\left\langle\varphi_{n}\right\rangle_{n<\omega}$ is such then:

(i) the limit system $\varphi=\bigcup_{n} \varphi_{n}=\left\langle T_{s}\right\rangle_{s \in 2<\omega}$ satisfies (*) of Definition 6.1 on the whole domain of strings $s \in 2^{<\omega}$;

(ii) $T=\bigcap_{n} \bigcup_{s \in 2^{n}} T_{s}$ is a perfect tree in $\mathbf{P T}$ and $[T]=\bigcap_{n} \bigcup_{s \in 2^{n}}\left[T_{s}\right]$;

(iii) if $u \in 2^{<\omega}$ then $T(\rightarrow u)=T \cap T_{u}=\bigcap_{n \geq \ln (u)} \bigcup_{s \in 2^{n}, u \subseteq s} T_{s}$.

\section{Multiforcings and multitrees}

We'll systematically make use of finite support products of perfect tree forcings in this paper. The following definitions introduce suitable notation.

Call a multiforcing any map $\boldsymbol{\pi}:|\boldsymbol{\pi}| \rightarrow$ PTF, where $|\boldsymbol{\pi}|=\operatorname{dom} \boldsymbol{\pi} \subseteq \omega_{1} \times \omega$. Thus each set $\boldsymbol{\pi}(\xi, k),\langle\xi, k\rangle \in|\boldsymbol{\pi}|$, is a perfect tree forcing notion. Such a $\boldsymbol{\pi}$ is:

- small, if both $|\boldsymbol{\pi}|$ and each forcing $\boldsymbol{\pi}(\xi, k),\langle\xi, k\rangle \in|\boldsymbol{\pi}|$, are countable;

- special, if each $\boldsymbol{\pi}(\xi, k)$ is special in the sense of Definition 5.4;

- regular, if each $\boldsymbol{\pi}(\xi, k)$ is regular, in the sense of Definition 5.4.

Let $\mathbf{M F}$ be the set of all multiforcings.

Let a multitree be any map $\boldsymbol{p}:|\boldsymbol{p}| \rightarrow \mathbf{P T}$, such that $|\boldsymbol{p}|=\operatorname{dom} \boldsymbol{p} \subseteq \omega_{1} \times \omega$ is finite and each value $T_{\xi k}^{\boldsymbol{p}}=\boldsymbol{p}(\xi, k)$ is a tree in PT. In this case we define a cofinite-dimensional perfect cube in $2^{\omega_{1} \times \omega}$

$$
\begin{aligned}
{[\boldsymbol{p}] } & =\left\{x \in 2^{\omega_{1} \times \omega}: \forall\langle\xi, k\rangle \in|\boldsymbol{p}|\left(x(\xi, k) \in\left[T_{\xi k}^{\boldsymbol{p}}\right]\right)\right\}= \\
& =\left\{x \in 2^{\omega_{1} \times \omega}: \forall\langle\xi, k\rangle \in|\boldsymbol{p}| \forall m\left(x(\xi, k)\left\lceil m \in T_{\xi k}^{\boldsymbol{p}}\right)\right\} .\right.
\end{aligned}
$$

Let MT be the set of all multitrees. We order MT componentwise: $\boldsymbol{q} \leqslant \boldsymbol{p}(\boldsymbol{q}$ is stronger) iff $|\boldsymbol{p}| \subseteq|\boldsymbol{q}|$ and $T_{\xi k}^{\boldsymbol{q}} \subseteq T_{\xi k}^{\boldsymbol{p}}$ for all $\langle\xi, k\rangle \in|\boldsymbol{p}|$; this is equivalent to $[\boldsymbol{q}] \subseteq[\boldsymbol{p}]$, so that stronger multitrees correspond to smaller cubes. The weakest multitree $\boldsymbol{\Lambda} \in \mathbf{M T}$ is just the empty map; $|\boldsymbol{\Lambda}|=\varnothing$ and $[\boldsymbol{\Lambda}]=2^{\omega_{1} \times \omega}$.

Multitrees $\boldsymbol{p}, \boldsymbol{q}$ are somewhere almost disjoint, or s.a.d., if, for at least one pair of indices $\langle\xi, k\rangle \in|\boldsymbol{p}| \cap|\boldsymbol{q}|$, the trees $T_{\xi k}^{\boldsymbol{p}}, T_{\xi k}^{\boldsymbol{q}}$ are a.d., that is, $\left[T_{\xi k}^{\boldsymbol{p}}\right] \cap\left[T_{\xi k}^{\boldsymbol{q}}\right]=$ $\varnothing$, or equivalently, $T_{\xi k}^{p} \cap T_{\xi k}^{q}$ is finite. 
Corollary 7.1 (of Lemma 5.5(i)). If $\boldsymbol{\pi}$ is a regular multiforcing and multitrees $\boldsymbol{p}, \boldsymbol{q} \in \mathbf{M T}(\boldsymbol{\pi})$ are not s.a.d., then $\boldsymbol{p}, \boldsymbol{q}$ are compatible in $\mathbf{M T}(\boldsymbol{\pi})$, so that there is a multitree $\boldsymbol{r} \in \mathbf{M T}(\boldsymbol{\pi})$ with $\boldsymbol{r} \leqslant \boldsymbol{p}, \boldsymbol{r} \leqslant \boldsymbol{q}$.

If $\boldsymbol{\pi}$ is a multiforcing then a $\boldsymbol{\pi}$-multitree is any multitree $\boldsymbol{p}$ with $|\boldsymbol{p}| \subseteq|\boldsymbol{\pi}|$ and $T_{\xi k}^{\boldsymbol{p}} \in \boldsymbol{\pi}(\xi, k)$ for all $\langle\xi, k\rangle \in|\boldsymbol{p}|$. Let $\mathbf{M T}(\boldsymbol{\pi})$ be the set of all $\boldsymbol{\pi}$-multitrees; it is equal to the finite support product $\prod_{\langle\xi, k\rangle \in|\boldsymbol{\pi}|} \boldsymbol{\pi}(\xi, k)$.

The following is similar to Lemma 5.5(iii).

Lemma 7.2. If a multiforcing $\boldsymbol{\pi}$ is regular, $\xi \subseteq|\boldsymbol{\pi}|$ is finite, and $U_{1}, \ldots, U_{k}$ are finite collections of multitrees in $\mathbf{M T}(\boldsymbol{\pi})$ with $|\boldsymbol{p}|=\xi$ for all $\boldsymbol{p} \in \bigcup_{i} U_{i}$, then there is a finite set of multitrees $\boldsymbol{u}_{1}, \ldots, \boldsymbol{u}_{n} \in \mathbf{M T}(\boldsymbol{\pi})$ such that $\left|\boldsymbol{u}_{j}\right|=\xi, \forall j$,

$$
\cup_{\boldsymbol{p} \in U_{1}}[\boldsymbol{p}] \cap \ldots \cap \bigcup_{\boldsymbol{p} \in U_{k}}[\boldsymbol{p}]=\left[\boldsymbol{u}_{1}\right] \cup \ldots \cup\left[\boldsymbol{u}_{n}\right],
$$

and for any $U_{i}$ and $\boldsymbol{u}_{j}$, there is $\boldsymbol{p} \in U_{i}$ such that $\left[\boldsymbol{u}_{j}\right] \subseteq[\boldsymbol{p}]$.

We consider sets of the form $\mathbf{M T}(\boldsymbol{\pi})$ in the role of product forcing notions. A set $D \subseteq \mathbf{M T}(\boldsymbol{\pi})$ is:

- open in $\mathbf{M T}(\boldsymbol{\pi})$, if for any $\boldsymbol{p} \leqslant \boldsymbol{q}$ in $\mathbf{M T}(\boldsymbol{\pi}), \boldsymbol{q} \in D \Longrightarrow \boldsymbol{p} \in D$;

- dense in $\mathbf{M T}(\boldsymbol{\pi})$, if for any $\boldsymbol{p} \in \mathbf{M T}(\boldsymbol{\pi})$, there is $\boldsymbol{q} \in D, \boldsymbol{q} \leqslant \boldsymbol{p}$;

- pre-dense in $\mathbf{M T}(\boldsymbol{\pi})$, if the set $D^{\prime}=\{\boldsymbol{p} \in \mathbf{M T}(\boldsymbol{\pi}): \exists \boldsymbol{q} \in D(\boldsymbol{p} \leqslant \boldsymbol{q})\}$ is dense in $\mathbf{M T}(\boldsymbol{\pi})$.

Remark 7.3. As a forcing notion, each $\mathbf{M T}(\boldsymbol{\pi})$ adds an array $\left\langle x_{\xi k}\right\rangle_{\langle\xi, k\rangle \in|\boldsymbol{\pi}|}$ of reals, where each real $x_{\xi k} \in 2^{\omega}$ is a $\pi(\xi, k)$-generic real. Namely if a set $G \subseteq \mathbf{M T}(\boldsymbol{\pi})$ is generic over the ground set universe $\mathbf{V}$ then each factor

$$
G(\xi, k)=\left\{T_{\xi k}^{\boldsymbol{p}}: \boldsymbol{p} \in G \wedge\langle\xi, k\rangle \in|\boldsymbol{p}|\right\} \subseteq \boldsymbol{\pi}(\xi, k)
$$

(where $\langle\xi, k\rangle \in|\boldsymbol{\pi}|$ ) is accordingly a set $\boldsymbol{\pi}(\xi, k)$-generic over $\mathbf{V}$, the real $x_{\xi k}=$ $x_{\xi k}[G]=x[G(\xi, k)] \in 2^{\omega}$ is the only real satisfying $x_{\xi k} \in \bigcap_{T \in G(\xi, k)}[T]$, and $x_{\xi k}$ is $\boldsymbol{\pi}(\xi, k)$-generic over $\mathbf{V}$ as in Lemma 5.7.

The reals of the form $x_{\xi k}[G]$ will be called principal generic reals in $\mathbf{V}[G]$.

Definition 7.4. A componentwise union of multiforcings $\pi, \boldsymbol{\varphi}$ is a multiforcing $\boldsymbol{\pi} \cup^{\mathrm{cw}} \boldsymbol{Q}$ satisfying $\left|\left(\boldsymbol{\pi} \cup^{\mathrm{cw}} \boldsymbol{\varphi}\right)\right|=|\boldsymbol{\pi}| \cup|\boldsymbol{\varphi}|$ and

$$
\left(\boldsymbol{\pi} \cup^{\mathrm{cw}} \boldsymbol{\varphi}\right)(\xi, k)=\left\{\begin{array}{rlll}
\boldsymbol{\pi}(\xi, k), & \text { whenever } & \langle\xi, k\rangle \in|\boldsymbol{\pi}| \backslash|\boldsymbol{\varphi}| \\
\boldsymbol{\varphi}(\xi, k), & \text { whenever } & \langle\xi, k\rangle \in|\boldsymbol{\varphi}| \backslash|\boldsymbol{\pi}| \\
\boldsymbol{\pi}(\xi, k) \cup \boldsymbol{\varphi}(\xi, k), & \text { whenever } & \langle\xi, k\rangle \in|\boldsymbol{\pi}| \cap|\boldsymbol{\varphi}|
\end{array}\right.
$$

Similarly, if $\overrightarrow{\boldsymbol{\pi}}=\left\langle\boldsymbol{\pi}_{\alpha}\right\rangle_{\alpha<\lambda}$ is a sequence of multiforcings then define a multiforcing $\boldsymbol{\pi}=\bigcup^{\mathrm{cw}} \overrightarrow{\boldsymbol{\pi}}=\bigcup_{\alpha<\lambda}^{\mathrm{cw}} \boldsymbol{\pi}_{\alpha}$ so that $\quad|\boldsymbol{\pi}|=\bigcup_{\alpha<\lambda}\left|\boldsymbol{\pi}_{\alpha}\right|$ and if $\langle\xi, k\rangle \in|\boldsymbol{\pi}|$ then $\boldsymbol{\pi}(\xi, k)=\bigcup_{\alpha<\lambda,\langle\xi, k\rangle \in\left|\boldsymbol{\pi}_{\alpha}\right|} \boldsymbol{\pi}_{\alpha}(\xi, k)$. 


\section{Multisystems}

The next definition introduces multisystems, a multi version of the splitting/fusion technique of Section 6, whose intention is to define suitable multiforcings, as will be shown in Section 11 below.

Definition 8.1. A multisystem is any map $\varphi:|\varphi| \rightarrow$ FSS, such that $|\varphi| \subseteq$ $\omega_{1} \times \omega \times \omega$ is finite. This amounts to

(1) the map $h^{\varphi}(\xi, k, m)=\operatorname{hgt}(\boldsymbol{\varphi}(\xi, k, m)):|\boldsymbol{\varphi}| \rightarrow \omega$, and

(2) the finite collection of trees $T_{\xi k, m}^{\varphi}(s)=\boldsymbol{\varphi}(\xi, k, m)(s)$, where $\langle\xi, k, m\rangle \in$ $|\boldsymbol{\varphi}|$ and $s \in 2^{\leq h^{\varphi}(\xi, k, m)}$, such that if $\langle\xi, k, m\rangle \in|\boldsymbol{\varphi}|$ then $\boldsymbol{\varphi}(\xi, k, m)=$ $\left\langle T_{\xi k, m}^{\varphi}(s)\right\rangle_{s \in 2 \leq h \varphi(\xi, k, m)}$ is a finite splitting system in FSS.

If $\boldsymbol{\pi}$ is a multiforcing, $|\boldsymbol{\varphi}| \subseteq(|\boldsymbol{\pi}|) \times \omega$, and $\boldsymbol{\varphi}(\xi, k, m) \in \boldsymbol{\operatorname { F S S }}(\boldsymbol{\pi}(\xi, k))$ for all $\langle\xi, k, m\rangle \in|\boldsymbol{\varphi}|$ (or equivalently, $T_{\xi k, m}^{\varphi}(s) \in \boldsymbol{\pi}(\xi, k)$ whenever $\langle\xi, k, m\rangle \in \boldsymbol{\varphi}$ and $\left.s \in 2^{\leq h^{\varphi}(\xi, k, m)}\right)$, then say that $\boldsymbol{\varphi}$ is a $\boldsymbol{\pi}$-multisystem, $\boldsymbol{\varphi} \in \mathbf{M S}(\boldsymbol{\pi})$.

Let $\boldsymbol{\varphi}, \boldsymbol{\psi}$ be multisystems. Say that $\boldsymbol{\varphi}$ extends $\boldsymbol{\psi}$, symbolically $\boldsymbol{\psi} \preccurlyeq \boldsymbol{\varphi}$, if $|\boldsymbol{\psi}| \subseteq|\boldsymbol{\varphi}|$, and, for every $\langle\xi, k, m\rangle \in|\boldsymbol{\psi}|, \boldsymbol{\varphi}(\xi, k, m)$ extends $\boldsymbol{\psi}(\xi, k, m)$, that is, $h^{\varphi}(\xi, k, m) \geq h^{\psi}(\xi, k, m)$ and $T_{\xi k, m}^{\varphi}(s)=T_{\xi k, m}^{\psi}(s)$ for all $s \in 2^{\leq h^{\psi}}(\xi, k, m)$.

It will be demonstrated in Section 11 that a suitably increasing infinite sequence $\varphi_{0} \preccurlyeq \varphi_{1} \preccurlyeq \varphi_{2} \preccurlyeq \ldots$ of multisystems in some $\mathbf{M S}(\boldsymbol{\pi})$ leads to a "limit" multiforcing $\boldsymbol{\varphi}$ with $|\boldsymbol{\varphi}|=\bigcup_{n}\left|\boldsymbol{\varphi}_{n}\right|$, such that each factor $\boldsymbol{\varphi}(\xi, k),\langle\xi, k\rangle \in|\boldsymbol{\pi}|$, is filled in by trees $Q_{\xi k, m}, m<\omega$, in such a way, that the $(\xi, k, m)$-components of the systems $\boldsymbol{\varphi}_{n}$ are responsible for the construction of the tree $Q_{\xi k, m}$.

The next lemma introduces different ways to extend a given multisystem.

Say that a multisystem $\varphi$ is 2wise disjoint if $\left[T_{\xi k, m}^{\varphi}(s)\right] \cap\left[T_{\eta \ell, n}^{\varphi}(t)\right]=\varnothing$ for all triples $\langle\xi, k, m\rangle \neq\langle\eta, \ell, n\rangle$ in $|\varphi|$ and all $s \in 2^{h^{\varphi}(\xi, k, m)}$ and $t \in 2^{h^{\varphi}(\eta, \ell, n)}$.

Lemma 8.2. Let $\boldsymbol{\pi}$ be a multiforcing and $\varphi \in \mathbf{M S}(\boldsymbol{\pi})$.

(i) If $\langle\xi, k, m\rangle \in|\varphi|$ and $h=h^{\varphi}(\xi, k, m)$ then the extension $\boldsymbol{\psi}$ of $\boldsymbol{\varphi}$ by $h^{\psi}(\xi, k, m)=h+1$ and $T_{\xi k, m}^{\psi}\left(s^{\wedge} i\right)=T_{\xi k, m}^{\varphi}(s)(\rightarrow i)$ for all $s \in 2^{h}$ and $i=0,1$, belongs to $\mathbf{M S}(\boldsymbol{\pi})$ and $\boldsymbol{\varphi} \preccurlyeq \boldsymbol{\psi}$.

(ii) If $\langle\xi, k, m\rangle \notin|\varphi|$ then the extension $\boldsymbol{\psi}$ of $\boldsymbol{\varphi}$ by $|\boldsymbol{\psi}|=|\boldsymbol{\varphi}| \cup\{\langle\xi, k, m\rangle\}$, $h^{\psi}(\xi, k, m)=0$ and $T_{\xi k, m}^{\psi}(\Lambda)=T$, where $T \in \boldsymbol{\pi}(\xi, k)$ and $\Lambda$ is the empty string, belongs to $\mathbf{M S}(\boldsymbol{\pi})$ and $\boldsymbol{\varphi} \preccurlyeq \boldsymbol{\psi}$.

(iii) If $\langle\xi, k, m\rangle \in|\varphi|$ and a set $D \subseteq \boldsymbol{\pi}(\xi, k)$ is open dense in $\boldsymbol{\pi}(\xi, k)$ then there is a multisystem $\boldsymbol{\psi} \in \mathbf{M T}(\boldsymbol{\pi})$ such that $|\boldsymbol{\psi}|=|\boldsymbol{\varphi}|, \boldsymbol{\varphi} \preccurlyeq \boldsymbol{\psi}$, and $T_{\xi k, m}^{\psi}(s) \in D$ whenever $s \in 2^{h^{\psi}(\xi, k, m)}$. 
(iv) There is a 2wise disjoint $\boldsymbol{\psi} \in \mathbf{M T}(\boldsymbol{\pi})$ such that $|\boldsymbol{\psi}|=|\boldsymbol{\varphi}|$ and $\boldsymbol{\varphi} \preccurlyeq \boldsymbol{\psi}$.

Proof. To prove (iii) first use (i) to get a multisystem $\boldsymbol{\psi} \in \operatorname{MS}(\boldsymbol{\pi})$ with $\boldsymbol{\varphi} \preccurlyeq$ $\psi$ and $h^{\psi}(\xi, k, m)=h+1$, where $h=h^{\varphi}(\xi, k, m)$. Then replace each tree $T_{\xi k, m}^{\psi}(s)=\boldsymbol{\psi}(\xi, k, m)(s), s \in 2^{h+1}$, with a suitable tree $T^{\prime} \in D, T^{\prime} \subseteq T_{\xi k, m}^{\psi}(s)$.

To prove (iv) first apply (i) to get a multisystem $\boldsymbol{\psi} \in \mathbf{M S}(\boldsymbol{\pi})$ with $\boldsymbol{\varphi} \preccurlyeq \boldsymbol{\psi}$, $|\boldsymbol{\psi}|=|\boldsymbol{\varphi}|$, and $h^{\boldsymbol{\psi}}(\xi, k, m)=h^{\boldsymbol{\varphi}}(\xi, k, m)+1$ for all $\langle\xi, k, m\rangle \in \mid \boldsymbol{\varphi}$. Now if $\langle\xi, k, m\rangle \neq\langle\eta, \ell, n\rangle$ are triples in $|\varphi|$ and $s \in 2^{h^{\varphi}(\xi, k, m)+1}, t \in 2^{h^{\varphi}(\eta, \ell, n)+1}$, then, by Lemma 5.3(ii), there are trees $S \in \boldsymbol{\pi}(\xi, k)$ and $T \in \boldsymbol{\pi}(\eta, \ell)$ satisfying $[S] \cap[T]=\varnothing$ and $S \subseteq T_{\xi k, m}^{\psi}(s), T \subseteq T_{\eta \ell, n}^{\psi}(t)$. Replace the trees $T_{\xi k, m}^{\psi}(s)$, $T \subseteq T_{\eta \ell, n}^{\psi}(t)$ with resp. $S, T$. Iterate this shrinking construction for all triples $\langle\xi, k, m\rangle \neq\langle\eta, \ell, n\rangle$ and strings $s, t$ as above. 


\section{Refinements}

Here we consider refinements of perfect tree forcings and multiforcings, the key technical tool of definition of various forcing notions in this paper.

\section{Refining perfect tree forcings}

If $T \in \mathbf{P T}$ (a perfect tree) and $D \subseteq \mathbf{P T}$ then $X \subseteq$ fin $\bigcup D$ will mean that there is a finite set $D^{\prime} \subseteq D$ such that $T \subseteq \bigcup D^{\prime}$, or equivalently $[T] \subseteq \bigcup_{S \in D^{\prime}}[S]$.

Definition 9.1. Let $\mathbb{P}, \mathbb{Q} \in \mathbf{P T F}$ be perfect tree forcing notions. Say that $\mathbb{Q}$ is a refinement of $\mathbb{P}$ (symbolically $\mathbb{P} \sqsubset \mathbb{Q}$ ) if

(1) the set $\mathbb{Q}$ is dense in $\mathbb{P} \cup \mathbb{Q}$ : if $T \in \mathbb{P}$ then $\exists Q \in \mathbb{Q}(Q \subseteq T)$;

(2) if $Q \in \mathbb{Q}$ then $Q \subseteq \subseteq^{\text {fin }} \cup \mathbb{P}$;

(3) if $Q \in \mathbb{Q}$ and $T \in \mathbb{P}$ then $[Q] \cap[T]$ is clopen in $[Q]$ and $T \nsubseteq Q$.

Lemma 9.2. (i) If $\mathbb{P} \sqsubset \mathbb{Q}$ and $S \in \mathbb{P}, T \in \mathbb{Q}$, then $[S] \cap[T]$ is meager in $[S]$, therefore $\mathbb{P} \cap \mathbb{Q}=\varnothing$ and $\mathbb{Q}$ is open dense in $\mathbb{P} \cup \mathbb{Q} ;$.

(ii) if $\mathbb{P} \sqsubset \mathbb{Q} \sqsubset \mathbb{R}$ then $\mathbb{P} \sqsubset \mathbb{R}$, thus $\sqsubset$ is a strict partial order;

(iii) if $\left\langle\mathbb{P}_{\alpha}\right\rangle_{\alpha<\lambda}$ is a ᄃ-increasing sequence in PTF and $0<\mu<\lambda$ then $\mathbb{P}=$ $\bigcup_{\alpha<\mu} \mathbb{P}_{\alpha} \sqsubset \mathbb{Q}=\bigcup_{\mu \leq \alpha<\lambda} \mathbb{P}_{\alpha} ;$

(iv) if $\left\langle\mathbb{P}_{\alpha}\right\rangle_{\alpha<\lambda}$ is a $\sqsubset$-increasing sequence in $\mathbf{P T F}$ and each $\mathbb{P}_{\alpha}$ is special then $\mathbb{P}=\bigcup_{\alpha<\lambda} \mathbb{P}_{\alpha}$ is a regular forcing in PTF ;

(v) in (iv), each $\mathbb{P}_{\gamma}$ is pre-dense in $\mathbb{P}=\bigcup_{\alpha<\lambda} \mathbb{P}_{\alpha}$.

Proof. (i) Otherwise there is a string $u \in S$ such that $S \uparrow_{u} \subseteq[T] \cap[S]$. But $S \uparrow_{u} \in \mathbb{P}$, which contradicts to $9.1(3)$.

(ii), (iii) Make use of (i) to establish 9.1(3).

(iv) To check the regularity let $S \in \mathbb{P}_{\alpha}, T \in \mathbb{P}_{\beta}, \alpha \leq \beta$. If $\alpha=\beta$ then, as $\mathbb{P}_{\alpha}$ is special, the trees $S, T$ are either a.d. or $\subseteq$-comparable by Lemma 5.5. If $\alpha<\beta$ then $[S] \cap[T]$ is clopen in $[T]$ by $9.1(3)$.

(v) Let $S \in \mathbb{P}_{\alpha}, \alpha \neq \gamma$. If $\alpha<\gamma$ then by 9.1(1) there is a tree $T \in \mathbb{P}_{\gamma}$, $T \subseteq S$. Now let $\gamma<\alpha$. Then $S \subseteq \subseteq^{\text {fin }} \cup \mathbb{P}_{\gamma}$ by 9.1(2), in particular, there is a tree $T \in \mathbb{P}_{\gamma}$ such that $[S] \cap[T] \neq \varnothing$. However $[S] \cap[T]$ is clopen in $[S]$ by $9.1(3)$. Therefore $S \uparrow_{u} \subseteq T$ for a string $u \in S$. Finally $S \uparrow_{u} \in \mathbb{P}_{\alpha}$ since $\mathbb{P}_{\alpha} \in \mathbf{P T F}$.

Note that if $\mathbb{P}, \mathbb{Q} \in \mathbf{P T F}$ and $\mathbb{P} \sqsubset \mathbb{Q}$ then a dense set $D \subseteq \mathbb{P}$ is not necessarily dense or even pre-dense in $\mathbb{P} \cup \mathbb{Q}$. Yet there is a special type of refinement which preserves at least pre-density. We modify the relation $\sqsubset$ as follows. 
Definition 9.3. Let $\mathbb{P}, \mathbb{Q} \in \mathbf{P T F}$ and $D \subseteq \mathbb{P}$. Say that $\mathbb{Q}$ locks $D$ over $\mathbb{P}$, symbolically $\mathbb{P} \sqsubset D \mathbb{Q}$, if $\mathbb{P} \sqsubset \mathbb{Q}$ holds and every tree $S \in \mathbb{Q}$ satisfies $S \subseteq$ fin $\cup D$. Then simply $\mathbb{P} \sqsubset \mathbb{Q}$ is equivalent to $\mathbb{P} \sqsubset_{\mathbb{P}} \mathbb{Q}$.

As we'll see now, a locked set has to be pre-dense both before and after the refinement. The additional importance of locking refinements lies in fact that, once established, it preserves under further simple refinements, that is, $\sqsubset D$ is transitive in a combination with $\sqsubset$ in the sense of (ii) of the following lemma:

Lemma 9.4. (i) If $\mathbb{P} \sqsubset D \mathbb{Q}$ then $D$ is pre-dense in $\mathbb{P} \cup \mathbb{Q}$, and if in addition $\mathbb{P}$ is regular then $D$ is pre-dense in $\mathbb{P}$ as well;

(ii) if $\mathbb{P} \sqsubset{ }_{D} \mathbb{Q} \sqsubset \mathbb{R}$ (note: the second $\sqsubset$ is not $\sqsubset_{D}$ !) then $\mathbb{P} \sqsubset{ }_{D} \mathbb{R}$;

(iii) if $\left\langle\mathbb{P}_{\alpha}\right\rangle_{\alpha<\lambda}$ is a ᄃ-increasing sequence in PTF, $0<\mu<\lambda$, and $\mathbb{P}=$ $\bigcup_{\alpha<\mu} \mathbb{P}_{\alpha} \sqsubset{ }_{D} \mathbb{P}_{\mu}$, then $\mathbb{P} \sqsubset D \mathbb{Q}=\bigcup_{\mu \leq \alpha<\lambda} \mathbb{P}_{\alpha}$.

Proof. (i) To see that $D$ is pre-dense in $\mathbb{P} \cup \mathbb{Q}$, let $T_{0} \in \mathbb{P} \cup \mathbb{Q}$. By 9.1(1), there is a tree $T \in \mathbb{Q}, T \subseteq T_{0}$. Then $T \subseteq$ fin $\bigcup D$, in particular, there is a tree $S \in D$ with $X=[S] \cap[T] \neq \varnothing$. However $X$ is clopen in $[T]$ by $9.1(3)$. Therefore, by Lemma 5.2 , there is a tree $T^{\prime} \in \mathbb{Q}$ with $\left[T^{\prime}\right] \subseteq X$, thus $T^{\prime} \subseteq S \in D$ and $T^{\prime} \subseteq T \subseteq T_{0}$. We conclude that $T_{0}$ is compatible with $S \in D$ in $\mathbb{P} \cup \mathbb{Q}$.

To see that $D$ is pre-dense in $\mathbb{P}$ (assuming $\mathbb{P}$ is regular), let $S_{0} \in \mathbb{P}$. It follows from the above that $S_{0}$ is compatible with some $S \in D$, hence, $S$ and $S_{0}$ are not absolutely incompatible. It remains to use Lemma 5.5(i).

To prove (ii) on the top of Lemma 9.2(ii), let $R \in \mathbb{R}$. Then $R \subseteq$ fin $\bigcup \mathbb{Q}$, but each $T \in \mathbb{Q}$ satisfies $T \subseteq$ fin $\bigcup D$. The same for (iii).

The existence of $\sqsubset_{D}$-refinements will be established below.

\section{Refining multiforcings}

Let $\boldsymbol{\pi}, \boldsymbol{\varphi}$ be multiforcings. Say that $\boldsymbol{\varphi}$ is an refinement of $\boldsymbol{\pi}$, symbolically $\boldsymbol{\pi} \sqsubset \boldsymbol{\varphi}$, if $|\boldsymbol{\pi}| \subseteq|\boldsymbol{Q}|$ and $\boldsymbol{\pi}(\xi, k) \sqsubset \boldsymbol{Q}(\xi, k)$ whenever $\langle\xi, k\rangle \in|\boldsymbol{\pi}|$.

Corollary 10.1 (of Lemma 9.2). If $\boldsymbol{\pi} \sqsubset \boldsymbol{\varphi} \sqsubset \boldsymbol{\rho}$ then $\boldsymbol{\pi} \sqsubset \boldsymbol{\rho}$.

If $\boldsymbol{\pi} \sqsubset \boldsymbol{\varphi}$ then the multiforcing $\mathbf{M T}(\boldsymbol{\varphi})$ is open dense in $\mathbf{M T}\left(\boldsymbol{\pi} \cup^{\mathrm{cw}} \boldsymbol{\varphi}\right)$.

Our next goal is to introduce a version of Definition 9.3 suitable for multiforcings; we expect an appropriate version of Lemma 9.4 to hold.

First of all, we accomodate the definition of the relation $\subseteq^{\text {fin }}$ in Section 9 for multitrees. Namely if $\boldsymbol{u}$ is a multitree and $\boldsymbol{D}$ a collection of multitrees, then $\boldsymbol{u} \subseteq{ }^{\text {fin }} \bigvee \boldsymbol{D}$ will mean that there is a finite set $\boldsymbol{D}^{\prime} \subseteq \boldsymbol{D}$ satisfying 1) $|\boldsymbol{v}|=|\boldsymbol{u}|$ for all $\boldsymbol{v} \in \boldsymbol{D}^{\prime}$, and 2) $[\boldsymbol{u}] \subseteq \bigcup_{\boldsymbol{v} \in \boldsymbol{D}^{\prime}}[\boldsymbol{v}]$. 
Definition 10.2. Let $\pi, \varphi$ be multiforcings, and $\pi \sqsubset \varphi$. Say that $\varphi$ locks a set $\boldsymbol{D} \subseteq \mathbf{M T}(\boldsymbol{\pi})$ over $\boldsymbol{\pi}$, symbolically $\boldsymbol{\pi} \sqsubset_{\boldsymbol{D}} \boldsymbol{Q}$ if the following condition holds:

$(*)$ if $\boldsymbol{p} \in \mathbf{M T}(\boldsymbol{\pi}), \boldsymbol{u} \in \mathbf{M T}(\boldsymbol{\varphi}),|\boldsymbol{u}| \subseteq|\boldsymbol{\pi}|,|\boldsymbol{u}| \cap|\boldsymbol{p}|=\varnothing$, then there is $\boldsymbol{q} \in \mathbf{M T}(\boldsymbol{\pi})$ such that $\boldsymbol{q} \leqslant \boldsymbol{p}$, still $|\boldsymbol{q}| \cap|\boldsymbol{u}|=\varnothing$, and $\boldsymbol{u} \subseteq^{\text {fin }} \bigvee \boldsymbol{D}_{\boldsymbol{q}}^{|\boldsymbol{u}|}$, where

$$
\boldsymbol{D}_{\boldsymbol{q}}^{|\boldsymbol{u}|}=\left\{\boldsymbol{u}^{\prime} \in \mathbf{M T}(\boldsymbol{\pi}):\left|\boldsymbol{u}^{\prime}\right|=|\boldsymbol{u}| \text { and } \boldsymbol{u}^{\prime} \cup \boldsymbol{q} \in \boldsymbol{D}\right\} .
$$

Note that if $\boldsymbol{p}, \boldsymbol{u}, \boldsymbol{D}, \boldsymbol{q}$ are as indicated then still $\boldsymbol{u} \cup \boldsymbol{q} \subseteq{ }^{\text {fin }} \bigvee \boldsymbol{D}$ holds via the finite set $\boldsymbol{D}^{\prime}=\left\{\boldsymbol{u}^{\prime} \cup \boldsymbol{q}: \boldsymbol{u}^{\prime} \in \boldsymbol{D}_{\boldsymbol{q}}^{|\boldsymbol{u}|}\right\} \subseteq \boldsymbol{D}$. Anyway the definition of $\complement_{\boldsymbol{D}}$ in 10.2 looks somewhat different and more complex then the definition of $\sqsubset_{D}$ in 9.3, which reflects the fact that finite-support products of forcing notions in PTF behave differently (and in more complex way) than single perfect-tree forcings. Accordingly, the next lemma, similar to Lemma 9.4, is way harder to prove.

Lemma 10.3. Let $\boldsymbol{\pi}, \boldsymbol{\varphi}, \boldsymbol{\sigma}$ be multiforcings and $\boldsymbol{D} \subseteq \mathbf{M T}(\boldsymbol{\pi})$. Then:

(i) if $\boldsymbol{\pi} \sqsubset{ }_{\boldsymbol{D}} \boldsymbol{\varphi}$ then $\boldsymbol{D}$ is dense in $\mathbf{M T}(\boldsymbol{\pi})$ and pre-dense in $\mathbf{M T}\left(\boldsymbol{\pi} \cup^{\mathrm{cw}} \boldsymbol{\varphi}\right)$;

(ii) if $\boldsymbol{\pi} \sqsubset{ }_{\boldsymbol{D}} \boldsymbol{\varphi}$ and $\boldsymbol{D} \subseteq \boldsymbol{D}^{\prime} \subseteq \mathbf{M T}(\boldsymbol{\pi})$ then $\boldsymbol{\pi} \sqsubset{ }_{\boldsymbol{D}^{\prime}} \boldsymbol{Q}$;

(iii) if $\boldsymbol{\pi}$ is regular, $\boldsymbol{\pi} \sqsubset \boldsymbol{D}_{i}$ P for $i=1, \ldots, n$, all sets $\boldsymbol{D}_{i} \subseteq \mathbf{M T}(\boldsymbol{\pi})$ are open dense in $\mathbf{M T}(\boldsymbol{\pi})$, and $\boldsymbol{D}=\bigcap_{i} \boldsymbol{D}_{i}$, then $\boldsymbol{\pi} \sqsubset \boldsymbol{D} \boldsymbol{Q}$;

(iv) if $\boldsymbol{D}$ is open dense in $\mathbf{M T}(\boldsymbol{\pi})$ and $\boldsymbol{\pi} \sqsubset{ }_{\boldsymbol{D}} \boldsymbol{\varphi} \sqsubset \boldsymbol{\sigma}$ then $\boldsymbol{\pi} \sqsubset_{\boldsymbol{D}} \boldsymbol{\sigma}$;

(v) if $\left\langle\boldsymbol{\pi}_{\alpha}\right\rangle_{\alpha<\lambda}$ is a ᄃ-increasing sequence in $\mathbf{M F}, 0<\mu<\lambda, \boldsymbol{\pi}=\bigcup_{\alpha<\mu}^{\mathrm{cw}} \boldsymbol{\pi}_{\alpha}$, $\boldsymbol{D}$ is open dense in $\mathbf{M T}(\boldsymbol{\pi})$, and $\boldsymbol{\pi} \sqsubset{ }_{\boldsymbol{D}} \boldsymbol{\pi}_{\mu}$, then $\boldsymbol{\pi} \sqsubset{ }_{\boldsymbol{D}} \boldsymbol{Q}=\bigcup_{\mu \leq \alpha<\lambda}^{\mathrm{cW}} \boldsymbol{\pi}_{\alpha}$.

Proof. (i) To check that $\boldsymbol{D}$ is pre-dense in $\mathbf{M T}\left(\boldsymbol{\pi} \cup \cup^{\mathrm{cw}} \boldsymbol{\varphi}\right)$, let $\boldsymbol{r} \in \mathbf{M T}\left(\boldsymbol{\pi} \cup^{\mathrm{cw}} \boldsymbol{\varphi}\right)$. Due to the product character of $\mathbf{M T}\left(\boldsymbol{\pi} \cup{ }^{\mathrm{cw}} \boldsymbol{\varphi}\right)$, we can assume that $|\boldsymbol{r}| \subseteq|\boldsymbol{\pi}|$. Let

$$
X=\left\{\langle\xi, k\rangle \in|\boldsymbol{r}|: T_{\xi k}^{r} \in \mathbf{M T}(\boldsymbol{\varphi})\right\}, \quad Y=\left\{\langle\xi, k\rangle \in|\boldsymbol{r}|: T_{\xi k}^{r} \in \mathbf{M T}(\boldsymbol{\pi})\right\} .
$$

Then $\boldsymbol{r}=\boldsymbol{u} \cup \boldsymbol{p}$, where $\boldsymbol{u}=\boldsymbol{r}\lceil X \in \mathbf{M T}(\boldsymbol{\varphi}), \boldsymbol{p}=\boldsymbol{r}\lceil Y \in \mathbf{M T}(\boldsymbol{\pi})$. As $\boldsymbol{\varphi}$ locks $\boldsymbol{D}$, there is a multitree $\boldsymbol{q} \in \mathbf{M T}(\boldsymbol{\pi})$ such that $\boldsymbol{q} \leqslant \boldsymbol{p},|\boldsymbol{q}| \cap|\boldsymbol{u}|=\varnothing$, and $\boldsymbol{u} \subseteq$ fin $\bigcup \boldsymbol{D}_{\boldsymbol{q}}^{|\boldsymbol{u}|}$. By an easy argument, there is a multitree $\boldsymbol{u}^{\prime} \in \boldsymbol{D}_{\boldsymbol{q}}^{|\boldsymbol{u}|}$ compatible with $\boldsymbol{u}$ in $\mathbf{M T}(\boldsymbol{\varphi})$; let $\boldsymbol{w} \in \mathbf{M T}(\boldsymbol{\varphi}), \boldsymbol{w} \leqslant \boldsymbol{u}, \boldsymbol{w} \leqslant \boldsymbol{u}^{\prime},|\boldsymbol{w}|=\left|\boldsymbol{u}^{\prime}\right|=|\boldsymbol{u}|$. Then the multitree $\boldsymbol{r}^{\prime}=\boldsymbol{w} \cup \boldsymbol{q} \in \mathbf{M T}(\boldsymbol{\pi} \vee \boldsymbol{\varphi})$ satisfies $\boldsymbol{r}^{\prime} \leqslant \boldsymbol{r}$ and $\boldsymbol{r}^{\prime} \leqslant \boldsymbol{u}^{\prime} \cup \boldsymbol{q} \in \boldsymbol{D}$.

To check that $\boldsymbol{D}$ is dense in $\mathbf{M T}(\boldsymbol{\pi})$, suppose that $\boldsymbol{p} \in \mathbf{M T}(\boldsymbol{\pi})$. Let $\boldsymbol{u}=\boldsymbol{\Lambda}$ (the empty multitree) in (*) of Definition 10.2 , so that $|\boldsymbol{u}|=\varnothing$ and $\boldsymbol{D}_{\boldsymbol{q}}^{|\boldsymbol{u}|}=\boldsymbol{D}$.

(ii) is obvious. To prove (iii), let $\boldsymbol{p} \in \mathbf{M T}(\boldsymbol{\pi}), \boldsymbol{u} \in \mathbf{M T}(\boldsymbol{\varphi}),|\boldsymbol{u}| \subseteq|\boldsymbol{\pi}|$, $|\boldsymbol{u}| \cap|\boldsymbol{p}|=\varnothing$. Iterating (*) for $\boldsymbol{D}_{i}, i=1, \ldots, n$, we find a multitree $\boldsymbol{q} \in \mathbf{M T}(\boldsymbol{\pi})$ such that $\boldsymbol{q} \leqslant \boldsymbol{p},|\boldsymbol{q}| \cap|\boldsymbol{u}|=\varnothing$, and $\boldsymbol{u} \subseteq{ }^{\text {fin }} \bigvee\left(\boldsymbol{D}_{i}\right)_{\boldsymbol{q}}^{|\boldsymbol{u}|}$ for all $i$, where

$$
\left(\boldsymbol{D}_{i}\right)_{\boldsymbol{q}}^{|\boldsymbol{u}|}=\left\{\boldsymbol{u}^{\prime} \in \mathbf{M T}(\boldsymbol{\pi}):\left|\boldsymbol{u}^{\prime}\right|=|\boldsymbol{u}| \text { and } \boldsymbol{u}^{\prime} \cup \boldsymbol{q} \in \boldsymbol{D}_{i}\right\} .
$$


Thus there are finite sets $U_{i} \subseteq\left(\boldsymbol{D}_{i}\right)_{\boldsymbol{q}}^{|\boldsymbol{u}|}$ such that $[\boldsymbol{u}] \subseteq \bigcup_{\boldsymbol{v} \in U_{i}}[\boldsymbol{v}]$ for all $i$. Using the regularity assumption and Lemma 7.2, we refine multitrees in $\bigcup_{i} U_{i}$, getting a finite set $W \subseteq \mathbf{M T}(\boldsymbol{\pi})$ such that still $|\boldsymbol{w}|=|\boldsymbol{u}|$ for all $\boldsymbol{w} \in W$, $\bigcap_{i} \bigcup_{\boldsymbol{v} \in U_{i}}[\boldsymbol{v}]=\bigcup_{\boldsymbol{w} \in W}[\boldsymbol{w}]$, and if $i=1, \ldots, n$ and $\boldsymbol{w} \in W$ then $[\boldsymbol{w}] \subseteq[\boldsymbol{v}]$ for some $\boldsymbol{v} \in U_{i}$ - therefore $\boldsymbol{w} \cup \boldsymbol{q} \in \boldsymbol{D}_{i}$. We conclude that if $\boldsymbol{w} \in W$ then $\boldsymbol{w} \cup \boldsymbol{q} \in \boldsymbol{D}$, hence $\boldsymbol{w} \in \boldsymbol{D}_{\boldsymbol{q}}^{|\boldsymbol{u}|}$. Thus $W \subseteq \boldsymbol{D}_{\boldsymbol{q}}^{|\boldsymbol{u}|}$. However $[\boldsymbol{u}] \subseteq \bigcup_{\boldsymbol{w} \in W}[\boldsymbol{w}]$ by the choice of $W$. We conclude that $\boldsymbol{u} \subseteq{ }^{\text {fin }} \bigvee \boldsymbol{D}_{\boldsymbol{q}}^{|\boldsymbol{u}|}$, as required.

(iv) It follows from Corollary 10.1 that $\boldsymbol{\pi} \sqsubset \boldsymbol{\sigma}$, hence it remains to check that $\boldsymbol{\sigma}$ locks $\boldsymbol{D}$ over $\boldsymbol{\pi}$. Assume that $\boldsymbol{u} \in \mathbf{M T}(\boldsymbol{\sigma}),|\boldsymbol{u}| \subseteq|\boldsymbol{\pi}|, \boldsymbol{p} \in \mathbf{M T}(\boldsymbol{\pi})$, $|\boldsymbol{u}| \cap|\boldsymbol{p}|=\varnothing$. As $\boldsymbol{\varphi} \sqsubset \boldsymbol{\sigma}$, there is a finite set $U \subseteq \mathbf{M T}(\boldsymbol{\varphi})$ such that $|\boldsymbol{v}|=|\boldsymbol{u}|$ for all $\boldsymbol{v} \in U$, and $[\boldsymbol{u}] \subseteq \bigcup_{\boldsymbol{v} \in U}[\boldsymbol{v}]$. As $\boldsymbol{\pi} \sqsubset_{\boldsymbol{D}} \boldsymbol{\varphi}$, by iterated application of (*) of Definition 10.2, we get a multitree $\boldsymbol{q} \in \mathbf{M T}(\boldsymbol{\pi})$ such that $\boldsymbol{q} \leqslant \boldsymbol{p}$, still $|\boldsymbol{q}| \cap|\boldsymbol{u}|=\varnothing$, and if $\boldsymbol{v} \in U$ then $\boldsymbol{v} \subseteq{ }^{\text {fin }} \bigvee \boldsymbol{D}_{\boldsymbol{q}}^{|\boldsymbol{u}|}$, where

$$
\boldsymbol{D}_{\boldsymbol{q}}^{|\boldsymbol{u}|}=\left\{\boldsymbol{v}^{\prime} \in \mathbf{M T}(\boldsymbol{\pi}):\left|\boldsymbol{v}^{\prime}\right|=|\boldsymbol{v}|=|\boldsymbol{u}| \wedge \boldsymbol{v}^{\prime} \cup \boldsymbol{q} \in \boldsymbol{D}\right\}
$$

Note finally that $\boldsymbol{u} \subseteq{ }^{\text {fin }} \bigvee U$ by construction, hence $\boldsymbol{u} \subseteq{ }^{\text {fin }} \bigvee \boldsymbol{D}_{\boldsymbol{q}}^{|\boldsymbol{u}|}$ as well.

(v) We have to check that $\boldsymbol{\varphi}$ locks $\boldsymbol{D}$ over $\boldsymbol{\pi}$. Let $\boldsymbol{u} \in \mathbf{M T}(\boldsymbol{\varphi}),|\boldsymbol{u}| \subseteq|\boldsymbol{\pi}|$, $\boldsymbol{p} \in \mathbf{M T}(\boldsymbol{\pi}),|\boldsymbol{u}| \cap|\boldsymbol{p}|=\varnothing$. As above, there is a finite set $U \subseteq \mathbf{M T}\left(\boldsymbol{\pi}_{\mu}\right)$ such that $|\boldsymbol{v}|=|\boldsymbol{u}|$ for all $\boldsymbol{v} \in U$ and $[\boldsymbol{u}] \subseteq \bigcup_{\boldsymbol{v} \in U}[\boldsymbol{v}]$. And so on as in the proof of (iv).

\section{Generic refinement of a multiforcing}

Here we introduce a construction, due to Jensen in its original form, which implies the existence of refinements of forcings and multiforcings, of types $\sqsubset_{D}$ and $\sqsubset_{\boldsymbol{D}}$.

Definition 11.1. 1. Suppose that $\boldsymbol{\pi}$ is a small multiforcing, and $\mathfrak{M} \in \mathrm{HC}$ is any set. (Recall that $\mathrm{HC}=$ all hereditarily countable sets.) This is the input.

2. The set $\mathfrak{M}^{+}$of all sets $X \in \mathrm{HC}, \in$-definable in $\mathrm{HC}$ by formulas with sets in $\mathfrak{M}$ as parameters, is still countable. Therefore there exists a $\preccurlyeq$-increasing sequence $\langle\boldsymbol{\varphi}(j)\rangle_{j<\omega}$ of multisystems $\boldsymbol{\varphi}(j) \in \mathbf{M S}(\boldsymbol{\pi}), \mathfrak{M}^{+}$-generic in the sense that it intersects any set $\Delta \subseteq \mathbf{M S}(\boldsymbol{\pi}), \Delta \in \mathfrak{M}^{+}$, dense in $\mathbf{M S}(\boldsymbol{\pi})$. (The density means that for any $\boldsymbol{\psi} \in \mathbf{M S}(\boldsymbol{\pi})$ there is a multisystem $\varphi \in \Delta$ with $\boldsymbol{\psi} \preccurlyeq \boldsymbol{\varphi}$.)

Let us fix any such a $\mathfrak{M}^{+}$-generic sequence $\mathbb{\phi}=\langle\boldsymbol{\varphi}(j)\rangle_{j<\omega}$.

3. Suppose that $\langle\xi, k\rangle \in|\boldsymbol{\pi}|$ and $m<\omega$. In particular, the sequence $\mathbb{d}$ intersects every (dense by Lemma 8.2(i),(ii)) set of the form

$$
\Delta_{\xi k m h}=\left\{\boldsymbol{\varphi} \in \mathbf{M S}(\boldsymbol{\pi}): h^{\varphi}(\xi, k, m) \geq h\right\} \in \mathfrak{M}^{+}, \quad \text { where } h<\omega .
$$

Hence a tree $\boldsymbol{T}_{\xi k, m}^{\circledR}(s) \in \boldsymbol{\pi}(\xi, k)$ can be associated to any $s \in 2^{<\omega}$, such that, for all $j$, if $\langle\xi, k, m\rangle \in|\boldsymbol{\varphi}(j)|$ and $\ln (s) \leq h^{\varphi(j)}(\xi, k, m)$ then $T_{\xi k, m}^{\varphi(j)}(s)=\boldsymbol{T}_{\xi k, m}^{\Phi p}(s)$. 
4. Then it follows from Lemma 6.3 that each set $\boldsymbol{Q}_{\xi k, m}^{\natural}=\bigcap_{h} \bigcup_{s \in 2^{h}} \boldsymbol{T}_{\xi k, m}^{\bowtie}(s)$ is a tree in PT (not necessarily in $\boldsymbol{\pi}(\xi, k)$ ), as well as the trees

$$
\boldsymbol{Q}_{\xi k, m}^{\natural \triangleright}(s)=\bigcap_{n \geq \ln (s)} \bigcup_{t \in 2^{n}, s \subseteq t} \boldsymbol{T}_{\xi k, m}^{\bowtie \triangleright}(t),
$$

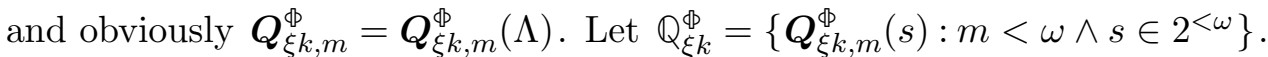

5. If $\langle\xi, k\rangle \in|\boldsymbol{\pi}|$ then let $\boldsymbol{Q}(\xi, k)=\mathbb{Q}_{\xi k}^{\Phi}=\left\{\boldsymbol{Q}_{\xi k, m}^{\circledR p}(s): m<\omega \wedge s \in 2^{<\omega}\right\}$.

6. Finally if $\boldsymbol{Q}=\boldsymbol{Q}[\mathbb{\mathrm { B }}]$ is obtained this way from an $\mathfrak{M}^{+}$-generic sequence $₫$ of multisystems in $\mathbf{M S}(\boldsymbol{\pi})$, then $\boldsymbol{Q}$ is called an $\mathfrak{M}$-generic refinement of $\boldsymbol{\pi}$.

Proposition 11.2 (by the countability of $\mathfrak{M}^{+}$). If $\boldsymbol{\pi}$ is a small multiforcing and $\mathfrak{M} \in \mathrm{HC}$ then there is an $\mathfrak{M}$-generic refinement $\boldsymbol{\varphi}$ of $\boldsymbol{\pi}$.

Theorem 11.3. If $\boldsymbol{\pi}$ is a small multiforcing, a set $\mathfrak{M} \in \mathrm{HC}$ contains $\boldsymbol{\pi}$, $|\boldsymbol{\pi}| \subseteq \mathfrak{M}$, and $\boldsymbol{\varphi}$ is an $\mathfrak{M}$-generic refinement of $\boldsymbol{\pi}$, then:

(i) $\boldsymbol{Q}$ is a small special multiforcing, $|\boldsymbol{Q}|=|\boldsymbol{\pi}|$, and $\boldsymbol{\pi} \sqsubset \boldsymbol{Q}$;

(ii) if $\langle\xi, k\rangle \in|\boldsymbol{\pi}|$ and a set $D \in \mathfrak{M}, D \subseteq \boldsymbol{\pi}(\xi, k)$ is pre-dense in $\boldsymbol{\pi}(\xi, k)$ then $\boldsymbol{\pi}(\xi, k) \sqsubset{ }_{D} \boldsymbol{\varphi}(\xi, k) ;$

(a) if $\langle\xi, k\rangle \in|\boldsymbol{\pi}|, m<\omega$, and $s \in 2^{<\omega}$ then $\boldsymbol{Q}_{\xi k, m}^{\llbracket p}(s)=\boldsymbol{Q}_{\xi k, m}^{\llbracket p}(\rightarrow s)$;

(b) if $\langle\xi, k\rangle \in|\boldsymbol{\pi}|, m<\omega$, and $s \in 2^{<\omega}$ then $\boldsymbol{Q}_{\xi k, m}^{\circledR}(s) \subseteq \boldsymbol{T}_{\xi k, m}^{\llbracket p}(s)$;

(c) if $\langle\xi, k\rangle \in|\boldsymbol{\pi}|, m<\omega$, and strings $t^{\prime} \neq t$ in $2^{<\omega}$ are $\subseteq$-incomparable then $\left[\boldsymbol{Q}_{\xi k, m}^{\natural \mathrm{p}}\left(t^{\prime}\right)\right] \cap\left[\boldsymbol{Q}_{\xi k, m}^{\natural}(t)\right]=\left[\boldsymbol{T}_{\xi k, m}^{\natural}\left(t^{\prime}\right)\right] \cap\left[\boldsymbol{T}_{\xi k, m}^{\llbracket \mathrm{p}}(t)\right]=\varnothing ;$

(d) if $\langle\xi, k, m\rangle \neq\langle\eta, \ell, n\rangle$ then $\left[\boldsymbol{Q}_{\xi k, m}^{\llbracket}\right] \cap\left[\boldsymbol{Q}_{\eta \ell, n}^{\llbracket}\right]=\varnothing$;

(e) if $\langle\xi, k\rangle \in|\boldsymbol{\pi}|, S \in \boldsymbol{\varphi}(\xi, k)$ and $T \in \boldsymbol{\pi}(\xi, k)$ then $[S] \cap[T]$ is clopen in $[S]$ and $T \nsubseteq S$, in particular, $\boldsymbol{\pi}(\xi, k) \cap \varphi(\xi, k)=\varnothing ;$

(f) if $\langle\xi, k\rangle \in|\boldsymbol{\pi}|$ then the set $\boldsymbol{\varphi}(\xi, k)$ is open dense in $\boldsymbol{\varphi}(\xi, k) \cup \boldsymbol{\pi}(\xi, k)$.

If in addition $\boldsymbol{\pi}=\bigcup_{\alpha<\lambda}^{\mathrm{cw}} \boldsymbol{\pi}_{\alpha}$, where $\lambda<\omega_{1},\left\langle\boldsymbol{\pi}_{\alpha}\right\rangle_{\alpha<\lambda}$ is a $\sqsubset$-increasing sequence of small special multiforcings, and $\mathfrak{M}$ contains $\left\langle\boldsymbol{\pi}_{\alpha}\right\rangle_{\alpha<\lambda}$ and all $\alpha<\lambda$, then

(iii) if $\alpha<\lambda$ then $\boldsymbol{\pi}_{\alpha} \sqsubset \boldsymbol{\varphi}$.

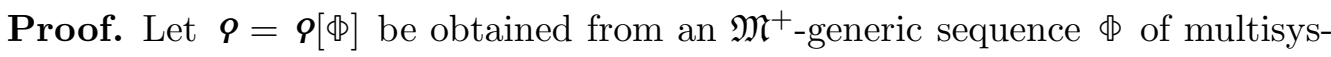
tems in $\mathbf{M S}(\boldsymbol{\pi})$, as above. We argue in the notation of Definition 11.1.

If $\langle\xi, k\rangle \in|\boldsymbol{\pi}|$ and $m<\boldsymbol{\omega}$ then by construction the system of trees $\boldsymbol{T}_{\xi k, m}^{\circledR}(s) \in$ $\boldsymbol{\pi}(\xi, k), s \in 2^{<\omega}$, satisfies $6.1(*)$ on the whole domain $s \in 2^{<\omega}$. This leads to (a), (b) (essentially corollaries of Lemma 6.3) and (c).

To prove $(\mathrm{d})$ note that the set $\Delta$ of all 2 wise disjoint multisystems $\varphi$ such that $|\boldsymbol{\varphi}|$ contains both $\langle\xi, k, m\rangle$ and $\langle\eta, \ell, n\rangle$, is dense in $\mathbf{M S}(\boldsymbol{\pi})$ by Lemma 8.2, 
and obviously $\Delta \in \mathfrak{M}^{+}$. Therefore there is $j<\omega$ such that $\varphi(j) \in \Delta$. Let $h=h^{\varphi(j)}(\xi, k, m)$ and $h^{\prime}=h^{\varphi(j)}(\eta, \ell, n)$. Then the sets

$$
A=\bigcup_{s \in 2^{h}}\left[T_{\xi k, m}^{\boldsymbol{\varphi}(j)}(s)\right]=\bigcup_{s \in 2^{h}}\left[\boldsymbol{T}_{\xi k, m}^{\natural}(s)\right], B=\bigcup_{t \in 2^{h^{\prime}}}\left[T_{\xi \ell, n}^{\varphi(j)}(t)\right]=\bigcup_{t \in 2^{h^{\prime}}}\left[\boldsymbol{T}_{\xi \ell, n}^{\bowtie p}(t)\right]
$$

are disjoint as $\boldsymbol{\varphi}(j) \in \Delta$. However $\left[\boldsymbol{Q}_{\xi k, m}^{\natural}\right] \subseteq A$ and $\left[\boldsymbol{Q}_{\eta \ell, n}^{\llbracket \triangleright}\right] \subseteq B$.

(i) It follows that the sets $\boldsymbol{\varphi}(\xi, k)=\mathbb{Q}_{\xi k}^{\mathbb{\Phi}}$ are special PTFs (Definition 5.4), and hence $\boldsymbol{Q}$ is a small special multiforcing, as in (i), and $|\boldsymbol{Q}|=|\boldsymbol{\pi}|$.

(e) To prove the clopenness claim, note that the set $\Delta$ of all multisystems $\boldsymbol{\varphi} \in \mathbf{M S}(\boldsymbol{\pi})$ such that $\langle\xi, k, m\rangle \in|\varphi|$ and if $s \in 2^{h}$, where $h=h^{\varphi}(\xi, k, m)$, then either $T_{\xi k, m}^{\varphi}(s) \subseteq T$ or $\left[T_{\xi k, m}^{\varphi}(s)\right] \cap[T]=\varnothing$, is dense. To prove $T \nsubseteq S$, the set $\Delta^{\prime}$ of all multisystems $\boldsymbol{\varphi} \in \mathbf{M S}(\boldsymbol{\pi})$ such that $\langle\xi, k, m\rangle \in|\varphi|$ and $T \not \bigcup_{s \in 2^{h}} T_{\xi k, m}^{\varphi}(s)$, where $h=h^{\varphi}(\xi, k, m)$, is dense. Note that $\Delta, \Delta^{\prime} \in \mathfrak{M}^{+}$and argue as above.

(f) Density. If $T \in \boldsymbol{\pi}(\xi, k)$ then the set $\Delta(T)$ of all multisystems $\boldsymbol{\varphi} \in \mathbf{M S}(\boldsymbol{\pi})$, such that $T_{\xi k, m}^{\varphi}(\Lambda)=T$ for some $m$, is dense in $\mathbf{M S}(\boldsymbol{\pi})$ by Lemma 8.2(ii), therefore $\varphi(j) \in \Delta(T)$ for some $j$. Then $\boldsymbol{T}_{\xi k, m}^{\circledR}(\Lambda)=T$ for some $m<\omega$. However $\boldsymbol{Q}_{\xi m, k}^{\natural}(\Lambda) \subseteq \boldsymbol{T}_{\xi k, m}^{\triangleleft \downarrow}(\Lambda)$. Openness. Suppose that $S \in \boldsymbol{Q}(\xi, k), T \in$ $\boldsymbol{\varphi}(\xi, k) \cup \boldsymbol{\pi}(\xi, k), T \subseteq S$. Then $T \notin \boldsymbol{\pi}(\xi, k)$ by (e). Therefore $T \in \boldsymbol{\varphi}(\xi, k)$.

(i), continuation. To establish $\boldsymbol{\pi} \sqsubset \boldsymbol{\varphi}$, let $\langle\xi, k\rangle \in|\boldsymbol{\pi}|$. We have to prove that $\boldsymbol{\pi}(\xi, k) \sqsubset \boldsymbol{\varphi}(\xi, k)$. This comes down to conditions (1), (2), (3) of Definition 9.1, of which (1) follows from (f) and (3) from (e), and (2) is obvious since $\boldsymbol{Q}_{\xi k, m}^{\natural p}(s) \subseteq$ $\boldsymbol{T}_{\xi k, m}^{\bowtie}(s) \in \boldsymbol{\pi}(\xi, k)$ for all $m$.

(ii) As $\boldsymbol{\pi} \sqsubset \boldsymbol{\varphi}$ has been checked, it remains to prove $\boldsymbol{Q}_{\xi k, m}^{\circledR \triangleright} \subseteq^{\text {fin }} \cup D$ for all $m$. It follows from the pre-density of $D$ that the set $D^{\prime}=\{T \in \boldsymbol{\pi}(\xi, k)$ : $\exists S \in D(T \subseteq S)\}$ is open dense in $\pi(\xi, k)$, and still $D^{\prime} \in \mathfrak{M}^{+}$. Then the set $\Delta \in \mathfrak{M}^{+}$of all multisystems $\varphi \in \mathbf{M S}(\boldsymbol{\pi})$ such that $\langle\xi, k, m\rangle \in|\boldsymbol{\varphi}|$ and $T_{\xi k, m}^{\varphi}(s) \in D$ for all $s \in h^{\varphi}(\xi, k, m)$, is dense in $\mathbf{M S}(\boldsymbol{\pi})$ by Lemma 8.2(iii). Thus $\boldsymbol{\varphi}(j) \in \Delta$ for some $j$, which witnesses $\boldsymbol{Q}_{\xi k, m}^{\natural} \subseteq^{\text {fin }} \cup D$.

(iii) We have to prove that $\boldsymbol{\pi}_{\alpha}(\xi, k) \sqsubset \boldsymbol{\varphi}(\xi, k)$ whenever $\langle\xi, k\rangle \in\left|\boldsymbol{\pi}_{\alpha}\right|$. And as $\boldsymbol{\pi}(\xi, k) \sqsubset \boldsymbol{\varphi}(\xi, k)$ has been checked, it suffices to prove that $\boldsymbol{Q}_{\xi k, m}^{\Phi} \subseteq^{\text {fin }} \bigcup \boldsymbol{\pi}_{\alpha}(\xi, k)$ for all $m$. However $D=\boldsymbol{\pi}_{\alpha}(\xi, k)$ is pre-dense in $\boldsymbol{\pi}(\xi, k)$ by Lemma $9.2(\mathrm{v})$, and still $D \in \mathfrak{M}^{+}$, hence we can refer to (ii).

Corollary 11.4. In the assumptions of Proposition 11.2, if $|\boldsymbol{\pi}| \subseteq Z \subseteq \omega_{1} \times \omega$ and $Z$ is at most countable then there is a small special multiforcing $\boldsymbol{Q}$ such that $|\boldsymbol{\varphi}|=Z$ and $\pi \sqsubset \boldsymbol{\varphi}$.

Proof. If $|\boldsymbol{\pi}|=Z$ then let $\mathfrak{M}$ be any countable set containing $\boldsymbol{\pi}$, pick $\boldsymbol{\varphi}$ by Proposition 11.2, and apply Theorem 11.3. If $|\boldsymbol{\pi}| \varsubsetneqq Z$ then we trivially extend the construction by $\boldsymbol{Q}(\xi, k)=\mathbb{P}_{\text {coh }}$ (see Example 5.1) for all $\langle\xi, k\rangle \in Z \backslash|\boldsymbol{\pi}|$. 
Corollary 11.5. Suppose that $\lambda<\omega_{1}$, and $\left\langle\mathbb{P}_{\alpha}\right\rangle_{\alpha<\lambda}$ is an $\sqsubset$-increasing sequence of countable special forcings in $\mathbf{P T F}$. Then there is a countable special forcing $\mathbb{Q} \in \mathbf{P T F}$ such that $\mathbb{P}_{\alpha} \sqsubset \mathbb{Q}$ for each $\alpha<\lambda$.

Proof. If $\alpha<\lambda$ then let a multiforcing $\boldsymbol{\pi}_{\alpha}$ be defined by $\left|\boldsymbol{\pi}_{\alpha}\right|=\{\langle 0,0\rangle\}$ and by $\boldsymbol{\pi}_{\alpha}(0,0)=\mathbb{P}_{\alpha}$. By Proposition 11.2 and Theorem 11.3 there is a multiforcing $\boldsymbol{\varphi}$ satisfying $|\boldsymbol{\varphi}|=\{\langle 0,0\rangle\}$ and $\boldsymbol{\pi}_{\alpha} \sqsubset \boldsymbol{\varphi}, \forall \alpha$. Let $\mathbb{Q}=\boldsymbol{\varphi}(0,0)$.

\section{Preservation of density}

This Section proves a special consequence of $\mathfrak{M}^{+}$-genericity of multiforcing refinements, the relation $\sqsubset$ of Definition 10.2 between a multiforcing and its refinement.

Theorem 12.1. In the assumptions of Theorem 11.3, if $\boldsymbol{D} \in \mathfrak{M}^{+}, \boldsymbol{D} \subseteq \mathbf{M T}(\boldsymbol{\pi})$, and $\boldsymbol{D}$ is open dense in $\mathbf{M T}(\boldsymbol{\pi})$, then $\boldsymbol{\pi} \sqsubset_{\boldsymbol{D}}$ 9 .

Proof. We suppose that $\boldsymbol{\varphi}=\boldsymbol{Q}[\mathbb{\$}]$ is obtained from an increasing $\mathfrak{M}^{+}$-generic sequence $\mathbb{Q}$ of multisystems in $\mathbf{M S}(\boldsymbol{\pi})$, as in Definition 11.1, and argue in the notation of 11.1 .

Suppose that $\boldsymbol{p} \in \mathbf{M T}(\boldsymbol{\pi}), \boldsymbol{u} \in \mathbf{M T}(\boldsymbol{\varphi}),|\boldsymbol{u}| \cap|\boldsymbol{p}|=\varnothing$, as in (*) of Definition 10.2 ; the extra condition $|\boldsymbol{u}| \subseteq|\boldsymbol{\pi}|$ holds automatically as we still have $|\boldsymbol{q}|=|\boldsymbol{\pi}|$. Let $X=|\boldsymbol{u}|, Y=|\boldsymbol{\pi}| \backslash X$. If $\langle\xi, k\rangle \in X$ then $T_{\xi k}^{\boldsymbol{u}}=\boldsymbol{Q}_{\xi k, m_{\xi k}}^{\mathrm{\phi}}\left(s_{\xi k}\right)$, where $m_{\xi k}<\omega$ and $s_{\xi k} \in 2^{<\omega}$. By obvious reasons we can assume that $s_{\xi k}=\Lambda$, hence $T_{\xi k}^{\boldsymbol{u}}=\boldsymbol{Q}_{\xi k, m_{\xi k}}^{\natural \downarrow}$, for all $\langle\xi, k\rangle \in X$.

Consider the set $\Delta$ of all multisystems $\varphi \in \mathbf{M S}(\boldsymbol{\pi})$ such that there is a number $H>0$ and a multitree $\boldsymbol{q} \in \mathbf{M S}(\boldsymbol{\pi})$ satisfying (1), (2), (3), (4) below.

(1) $|\boldsymbol{q}| \cap X=\varnothing$ and $\boldsymbol{q} \leqslant \boldsymbol{p}$;

(2) if $\langle\xi, k\rangle \in X$ then $\left\langle\xi, k, m_{\xi k}\right\rangle \in|\varphi|$;

(3) if $\langle\xi, k, m\rangle \in|\varphi|$ then $h^{\varphi}(\xi, k, m)=H$.

To formulate the last requirement, we need one more definition. Suppose that $\tau=\left\langle t_{\xi k}\right\rangle_{\langle\xi, k\rangle \in X}$ is a system of strings $\tau(\xi, k)=t_{\xi k} \in 2^{H}$, symbolically $\tau \in$ $\left(2^{H}\right)^{X}$. Define a multitree $\mathbf{s}(\boldsymbol{\varphi}, \tau) \in \mathbf{M T}(\boldsymbol{\pi})$ so that $|\mathbf{s}(\boldsymbol{\varphi}, \tau)|=X$ and $T_{\xi k}^{\mathbf{s}(\boldsymbol{\varphi}, \tau)}=$ $T_{\xi k, m_{\xi k}}^{\varphi}\left(t_{\xi k}\right)$ for all $\langle\xi, k\rangle \in X$. Note that $|\mathbf{s}(\boldsymbol{\varphi}, \tau)|=|\boldsymbol{u}|$, and hence the multitree $\mathbf{s}(\boldsymbol{\varphi}, \tau) \cup \boldsymbol{q}$ belongs to $\mathbf{M T}(\boldsymbol{\pi})$ as well. ${ }^{3}$ Now goes the last condition.

(4) If $\tau \in\left(2^{H}\right)^{X}$ then $\mathbf{s}(\boldsymbol{\varphi}, \tau) \cup \boldsymbol{q} \in \boldsymbol{D}$.

\footnotetext{
${ }^{3}$ Here, if $\boldsymbol{p}, \boldsymbol{q}$ are multitrees satisfying $|\boldsymbol{p}| \cap|\boldsymbol{q}|=\varnothing$ (disjoint domains), then $\boldsymbol{p} \cup \boldsymbol{q}$, a disjoint union, is a multitree such that $|\boldsymbol{p} \cup \boldsymbol{q}|=|\boldsymbol{p}| \cup|\boldsymbol{q}|$ and $T_{\xi k}^{\boldsymbol{p} \cup \boldsymbol{q}}=T_{\xi k}^{\boldsymbol{p}}$ whenever $\langle\xi, k\rangle \in|\boldsymbol{p}|$ but $T_{\xi k}^{p \cup \boldsymbol{q}}=T_{\xi k}^{\boldsymbol{q}}$ whenever $\langle\xi, k\rangle \in|\boldsymbol{q}|$.
} 
Lemma 12.2. The set $\Delta$ is dense in $\mathbf{M S}(\boldsymbol{\pi})$.

Proof (Lemma). Suppose that $\boldsymbol{\psi} \in \mathbf{M S}(\boldsymbol{\pi})$; we have to find a multisystem $\boldsymbol{\varphi} \in \mathbf{M S}(\boldsymbol{\pi})$ with $\boldsymbol{\psi} \preccurlyeq \boldsymbol{\varphi}$. First of all, by Lemma 8.2(i)(ii) we can assume that

(a) if $\langle\xi, k\rangle \in X$ then $\left\langle\xi, k, m_{\xi k}\right\rangle \in|\boldsymbol{\psi}|$;

(b) there is a number $g>0$ such that $h^{\psi}(\xi, k, m)=g$ for all $\langle\xi, k, m\rangle \in|\boldsymbol{\psi}|$.

Let $H=g+1$. Define $\chi \in \mathbf{M S}(\boldsymbol{\pi})$ so that $|\chi|=|\boldsymbol{\psi}|$, and $h^{\chi}(\xi, k, m)=H$, $T_{\xi k, m}^{\chi}\left(s^{\frown} i\right)=T_{\xi k, m}^{\psi}(s)(\rightarrow i)$ for all $\langle\xi, k, m\rangle \in|\boldsymbol{\psi}|$ and $s^{\frown} i \in 2^{H}$; then $\boldsymbol{\psi} \preccurlyeq \boldsymbol{\chi}$.

It follows from the open density of $\boldsymbol{D}$ that there is a multitree $\boldsymbol{q} \in \mathbf{M T}(\boldsymbol{\pi})$ satisfying (1), and a multisystem $\boldsymbol{\varphi} \in \mathbf{M S}(\boldsymbol{\pi})$ satisfying (4) and such that still $|\boldsymbol{\varphi}|=|\boldsymbol{\psi}|$ and $h^{\varphi}(\xi, k, m)=H$ for all $\langle\xi, k, m\rangle \in|\boldsymbol{\psi}|$, and in addition

(c) if $\langle\xi, k\rangle \in X$ and $s \in 2^{H}$ then $T_{\xi k, m_{\xi k}}^{\varphi}(s) \subseteq T_{\xi k, m_{\xi k}}^{\chi}(s)$;

(d) $T_{\xi k, m}^{\varphi}(s)=T_{\xi k, m}^{\chi}(s)$ for all applicable $\xi, k, m, s$ not covered by (c).

Namely to achieve (4) for one particular $\tau \in\left(2^{H}\right)^{X}$, consider the multitree $\boldsymbol{r}=\mathbf{s}(\chi, \tau) \cup \boldsymbol{p}$. There is a multitree $\boldsymbol{r}^{\prime} \in \boldsymbol{D}, \boldsymbol{r}^{\prime} \leqslant \boldsymbol{r}$. Let a new multisystem $\chi^{\prime}$ be obtained from $\chi$ by the reassignment $T_{\xi k, m_{\xi k}}^{\chi^{\prime}}(\tau(\xi, k))=T_{\xi k}^{r^{\prime}}$ for all $\langle\xi, k\rangle \in X$. To get the input for the next step, let $\boldsymbol{p}^{\prime}=\boldsymbol{r}^{\prime} \uparrow Y,{ }^{4}$ so that $\boldsymbol{r}^{\prime}=\mathbf{s}\left(\chi^{\prime}, \tau\right) \cup \boldsymbol{p}^{\prime} \in \boldsymbol{D}$.

Now consider another $\tau^{\prime} \in\left(2^{H}\right)^{X}$ and the multitree $\boldsymbol{r}^{\prime}=\mathbf{s}\left(\chi^{\prime}, \tau^{\prime}\right) \cup \boldsymbol{p}^{\prime}$. There is $\boldsymbol{r}^{\prime \prime} \in \boldsymbol{D}, \boldsymbol{r}^{\prime \prime} \leqslant \boldsymbol{r}^{\prime}$. Define $\chi^{\prime \prime}$ from $\chi^{\prime}$ by the reassignment $T_{\xi k, m_{\xi k}}^{\chi^{\prime \prime}}\left(\tau^{\prime}(\xi, k)\right)=$ $T_{\xi k}^{r^{\prime \prime}}$ for all $\langle\xi, k\rangle \in X$. Let $\boldsymbol{p}^{\prime \prime}=\boldsymbol{r}^{\prime \prime}\left\lceil Y\right.$, so that $\boldsymbol{r}^{\prime \prime}=\mathbf{s}\left(\chi^{\prime \prime}, \tau^{\prime}\right) \cup \boldsymbol{p}^{\prime \prime} \in \boldsymbol{D}$.

And so on. The final multisystem and multitree of this construction will be $\boldsymbol{\varphi}$ and $\boldsymbol{q}$ satisfying (1), (2), (3), (4). Note that $\boldsymbol{\psi} \preccurlyeq \boldsymbol{\varphi}$, as we only amend the $H$-th level of $\chi$ absent in $\boldsymbol{\psi}$.

$\square$ (Lemma)

Note that $\Delta$ is defined in HC using sets $\boldsymbol{D}, \boldsymbol{\pi}, \boldsymbol{p}, X$, and the map $\langle\xi, k\rangle \rightarrow$ $m_{\xi k}: X \rightarrow \omega$ as parameters. Now, $\boldsymbol{D}, \boldsymbol{\pi}$ belong to $\mathfrak{M}^{+}$straightforwardly, $X$ belongs to $\mathfrak{M}^{+}$since it is a finite subset of a set $|\boldsymbol{\pi}| \subseteq \mathfrak{M}$, and $\boldsymbol{p}$ belongs to $\mathfrak{M}^{+}$ by similar reasons. It follows that $\Delta$ belongs to $\mathfrak{M}^{+}$as well.

Therefore, by the lemma and the choice of $₫$, there is an index $j$ such that the multisystem $\varphi(j)$ belongs to $\Delta$, which is witnessed by a number $H>0$ and a multitree $\boldsymbol{q} \in \mathbf{M T}(\boldsymbol{\pi})$ satisfying (1), (2), (3), (4) for $\boldsymbol{\varphi}(j)$ instead of $\boldsymbol{\varphi}$. To prove that $\boldsymbol{u} \subseteq{ }^{\text {fin }} \bigvee \boldsymbol{D}_{\boldsymbol{q}}^{|\boldsymbol{u}|}$, note that the multitrees $\mathbf{s}(\boldsymbol{\varphi}(j), \tau) \cup \boldsymbol{q}, \tau \in\left(2^{H}\right)^{X}$, belong to $\boldsymbol{D}$ by (4), and easily $[\boldsymbol{u}] \subseteq \bigcup_{\tau \in\left(2^{H}\right)^{X}}[\mathbf{s}(\boldsymbol{\varphi}(j), \tau)]$.

\footnotetext{
${ }^{4}$ Here $\boldsymbol{r}^{\prime} \mid Y$ is the plain restriction of the function $\boldsymbol{r}^{\prime}:\left|\boldsymbol{r}^{\prime}\right| \rightarrow$ PT to the set $\left|\boldsymbol{r}^{\prime}\right| \cap Y$.
} 


\section{Structure of real names}

Here we turn to some details of the structure of reals in models of $\mathbf{M T}(\boldsymbol{\pi})$-generic type, $\boldsymbol{\pi}$ being a multiforcing. We are going focus on non-principal reals, i.e., those different from the principal generic reals $x_{\xi k}[G]$ (Remark 7.3). We'll work towards the goal of making every non-principal real to be non-generic with respect to each of the factor forcing notions $\boldsymbol{\pi}(\xi, k)$.

\section{Real names}

Our next goal is to introduce a suitable notation related to names of reals in the context of forcing notions of the form $\mathbf{M T}(\boldsymbol{\pi})$.

Definition 13.1. A real name is any set $\mathbf{c} \subseteq \mathbf{M T} \times(\omega \times \omega)$ such that the sets $K_{n i}^{\mathbf{c}}=\{\boldsymbol{p} \in \mathbf{M T}:\langle\boldsymbol{p}, n, i\rangle \in \mathbf{c}\}$ satisfy the following:

$(*)$ if $n, k, \ell<\omega, k \neq \ell$, and $\boldsymbol{p} \in K_{n k}^{\mathbf{c}}, \boldsymbol{q} \in K_{n \ell}^{\mathbf{c}}$, then $\boldsymbol{p}, \boldsymbol{q}$ are a.d..

A real name $\mathbf{c}$ is small if each set $K_{n i}^{\mathbf{c}}$ is at most countable - then the sets $\operatorname{dom} \mathbf{c}=\bigcup_{n, i} K_{n i}^{\mathbf{c}} \subseteq \mathbf{M T}$ and $|\mathbf{c}|=\bigcup_{n, i} \bigcup_{\boldsymbol{p} \in K_{n i}^{\mathbf{c}}}|\boldsymbol{p}| \subseteq \omega_{1} \times \omega$, and c itself, are countable, too.

Definition 13.2. Let $\mathbf{c}$ be a real name and $G \subseteq \mathbf{M T}$ a pairwise compatible set. Define the evaluation $\mathbf{c}[G] \in \omega^{\omega}$ so that $\mathbf{c}[G](n)=i$ iff:

- either $\exists \boldsymbol{p} \in G \exists \boldsymbol{q} \in K_{n i}^{\mathbf{c}}(\boldsymbol{p} \leqslant \boldsymbol{q})$ (recall that $\boldsymbol{p} \leqslant \boldsymbol{q}$ means $\boldsymbol{p}$ is stronger),

- or just $i=0$ and $\neg \exists \boldsymbol{p} \in G \exists \boldsymbol{q} \in \bigcup_{i} K_{n i}^{\mathbf{c}}(\boldsymbol{p} \leqslant \boldsymbol{q})$ (default case).

Definition 13.3. Let $\boldsymbol{\pi}$ be a multiforcing. A real name $\mathbf{c}$ is said to be a $\boldsymbol{\pi}$-real name if, in addition to $(*)$ above, the following condition holds:

(†) each set $K_{n}^{\mathbf{c}}=\bigcup_{i} K_{n i}^{\mathbf{c}}$ is pre-dense for $\mathbf{M T}(\boldsymbol{\pi})$, in the sense that the set

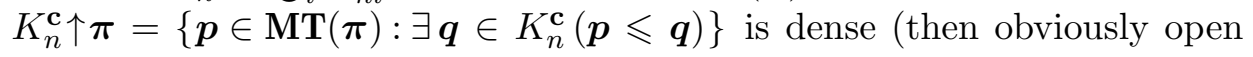
dense) in $\mathbf{M T}(\boldsymbol{\pi})$.

Generally speaking, we do not assume that $K_{n}^{\mathbf{c}} \subseteq \mathbf{M T}(\boldsymbol{\pi})$. However if, in addition to $(*),(\dagger)$ above, $K_{n}^{\mathbf{c}} \subseteq \mathbf{M T}(\boldsymbol{\pi})$ holds for all $n$, then say that $\mathbf{c}$ is a true $\boldsymbol{\pi}$-real name. Then each set $K_{n}^{\mathbf{c}}=\bigcup_{i} K_{n i}^{\mathbf{c}}$ is a pre-dense subset of $\mathbf{M T}(\boldsymbol{\pi})$.

Remark 13.4. Let $\boldsymbol{\pi}$ be a multiforcing, c be a $\boldsymbol{\pi}$-real name, and a set $G \subseteq$ $\operatorname{MT}(\boldsymbol{\pi})$ be $\mathbf{M T}(\boldsymbol{\pi})$-generic over the collection of all sets $K_{n}^{\mathbf{c}} \uparrow \boldsymbol{\pi}$ as in (†) (All of $K_{n}^{\mathbf{c}} \uparrow \boldsymbol{\pi}$ are dense by the choice of $\mathbf{c}$.) Then the "or" case in Definition 13.2 never

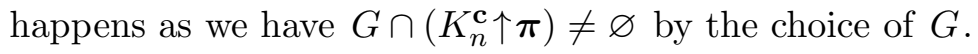


Remark 13.5. If $\boldsymbol{\pi}$ is a regular multiforcing then the notions of being a.d. and being incompatible in $\mathbf{M T}(\boldsymbol{\pi})$ are equivalent by Lemma 5.5(i), so that a true $\boldsymbol{\pi}$ real name is the same as a $\mathbf{M T}(\boldsymbol{\pi})$-name for an element of $\omega^{\omega}$ in the general theory of (unramified) forcing.

Example 13.6. If $\xi<\omega_{1}, k<\omega$, then $\dot{\boldsymbol{x}}_{\xi k}$ is a real name such that if $i=0,1$ then the set $C_{n i}^{\xi k}=K_{n i}^{\dot{\boldsymbol{x}}_{\xi k}}$ consists of a lone multitree $\boldsymbol{r}=\boldsymbol{r}_{n i}^{\xi k}$ with $|\boldsymbol{r}|=\{\langle\xi, k\rangle\}$ and $T_{\xi k}^{r}=\left\{t \in 2^{<\omega}: \ln (t) \leq n \vee t(n)=i\right\}$, and if $i \geq 2$ then $C_{n i}^{\xi k}=\varnothing$.

Remark 13.7. If $\boldsymbol{\pi} \in \mathbf{M T}$ and $\langle\xi, k\rangle \in|\boldsymbol{\pi}|$ then $\dot{\boldsymbol{x}}_{\xi k}$ is a $\boldsymbol{\pi}$-real name of the real $x_{\xi k}=x_{\xi k}[G] \in 2^{\omega}$, the $(\xi, k)$ th term of a $\mathbf{M T}(\boldsymbol{\pi})$-generic sequence $\left\langle x_{\xi k}[G]\right\rangle_{\langle\xi, k\rangle \in|\boldsymbol{\pi}|}$. That is, if $G \subseteq \mathbf{M T}(\boldsymbol{\pi})$ is generic then the real $x_{\xi k}[G]$ defined by 7.3 coincides with the real $\dot{\boldsymbol{x}}_{\xi k}[G]$ defined by 13.2 .

\section{Direct forcing}

The following definition of the direct forcing relation is not explicitly associated with any concrete forcing notion, but in fact the direct forcing relation (in all three instances) is compatible with any forcing notion of the form $\mathbf{M T}(\boldsymbol{\pi})$.

Let $\mathbf{c}$ be a real name. Let us say that a multitree $\boldsymbol{p}$ :

- directly forces $\mathbf{c}(n)=i$, where $n, i<\omega$, iff there is a multitree $\boldsymbol{q} \in K_{n i}^{\mathbf{c}}$ such that $\boldsymbol{p} \leqslant \boldsymbol{q}$ (meaning: $\boldsymbol{p}$ is stronger);

- directly forces $s \subset \mathbf{c}$, where $s \in \omega^{<\omega}$, iff for all $n<\operatorname{lh}(s), \boldsymbol{p}$ directly forces $\mathbf{c}(n)=i$, where $i=s(n)$;

- directly forces $\mathbf{c} \notin[T]$, where $T \in \mathbf{P T}$, iff there is a string $s \in \omega^{<\omega} \backslash T$ such that $\boldsymbol{p}$ directly forces $s \subset \mathbf{c}$.

Lemma 14.1. If $\boldsymbol{\pi}$ is a multiforcing and $\mathbf{c}$ is a $\boldsymbol{\pi}$-real name, $\boldsymbol{p} \in \mathbf{M T}(\boldsymbol{\pi})$, $\langle\xi, k\rangle \in|\boldsymbol{\pi}|, T \in \mathbf{P T}, n<\omega$, then

(i) there is a number $i<\omega$ and a multitree $\boldsymbol{q} \in \mathbf{M T}(\boldsymbol{\pi}), \boldsymbol{q} \leqslant \boldsymbol{p}$, which directly forces $\mathbf{c}(n)=i$;

(ii) there is a multitree $\boldsymbol{q} \in \mathbf{M T}(\boldsymbol{\pi}), \boldsymbol{q} \leqslant \boldsymbol{p}$, which directly forces $\mathbf{c} \notin[T(\rightarrow 0)]$ or directly forces $\mathbf{c} \notin[T(\rightarrow 1)]$.

Note that if $T \in \boldsymbol{\pi}(\xi, k)$ then the trees $T(\rightarrow i), i=0,1$ belong to $\boldsymbol{\pi}(\xi, k)$.

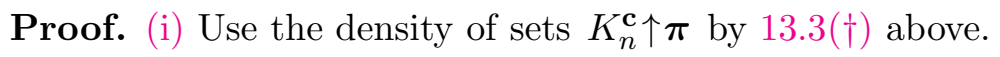

(ii) Let $r=\operatorname{stem}(T), n=\operatorname{lh}(r)$. By (i), there is a multitree $\boldsymbol{q} \in \mathbf{M T}(\boldsymbol{\pi})$, $\boldsymbol{p}^{\prime} \leqslant \boldsymbol{p}$, and, for any $m \leq n,-$ a number $i_{m}=0,1$, such that $\boldsymbol{q}$ directly forces $\mathbf{c}(m)=i_{m}, \forall m<n$. Let $s \in 2^{n+1}$ be defined by $s(m)=i_{m}$ for every $m \leq n$. Then $\boldsymbol{q}$ directly forces $s \subset \mathbf{c}$. On the other hand, $s$ cannot belong to both $T(\rightarrow 0)$ and $T(\rightarrow 1)$. 


\section{Locking real names}

The next definition extends Definition 10.2 to real names.

Definition 15.1. Assume that $\boldsymbol{\pi}, \varphi$ are multiforcings, $\mathrm{c}$ is a real name, and $\boldsymbol{\pi} \sqsubset \boldsymbol{\varphi}$. Say that $\boldsymbol{\varphi}$ locks c over $\boldsymbol{\pi}$, symbolically $\boldsymbol{\pi} \sqsubset_{\mathbf{c}} \boldsymbol{\varphi}$, if $\boldsymbol{\varphi}$ locks, over $\boldsymbol{\pi}$, each set $K_{n}^{\mathbf{c}} \uparrow \boldsymbol{\pi}$ defined in 13.3(†), in the sense of Definition 10.2.

Corollary 15.2 (of Theorem 12.1). In the assumptions of Theorem 11.3, if $\mathbf{c} \in \mathfrak{M}^{+}$and $\mathbf{c}$ is a $\boldsymbol{\pi}$-real name then $\boldsymbol{\pi} \sqsubset_{\mathbf{c}} \boldsymbol{Q}$.

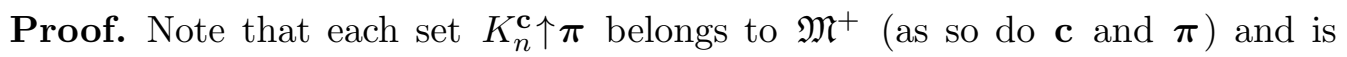
dense in $\mathbf{M T}(\boldsymbol{\pi})$, so it remains to apply Theorem 12.1.

Lemma 15.3. Let $\boldsymbol{\pi}, \boldsymbol{\varphi}, \boldsymbol{\sigma}$ be multiforcings and $\mathbf{c}$ be a real name. Then

(i) if $\boldsymbol{\pi} \sqsubset_{\mathbf{c}} \boldsymbol{\varphi}$ then $\mathbf{c}$ is a $\boldsymbol{\pi}$-real name and $a\left(\boldsymbol{\pi} \cup \cup^{\mathrm{cw}} \boldsymbol{Q}\right)$-real name;

(ii) if $\boldsymbol{\pi} \sqsubset_{\mathrm{c}} \boldsymbol{\varphi} \sqsubset \boldsymbol{\sigma}$ then $\boldsymbol{\pi} \sqsubset_{\mathrm{c}} \boldsymbol{\sigma}$;

(iii) if $\left\langle\boldsymbol{\pi}_{\alpha}\right\rangle_{\alpha<\lambda}$ is a ᄃ-increasing sequence in $\mathbf{M F}, 0<\mu<\lambda, \boldsymbol{\pi}=\bigcup_{\alpha<\mu}^{\mathrm{cw}} \boldsymbol{\pi}_{\alpha}$, and $\boldsymbol{\pi} \sqsubset_{\mathbf{c}} \boldsymbol{\pi}_{\mu}$, then $\boldsymbol{\pi} \sqsubset_{\mathbf{c}} \boldsymbol{\varphi}=\bigcup_{\mu \leq \alpha<\lambda}^{\mathrm{cw}} \boldsymbol{\pi}_{\alpha}$.

Proof. (i) By definition, we have $\boldsymbol{\pi} \sqsubset_{K_{n}^{\mathbf{c} \uparrow \boldsymbol{\pi}}} \boldsymbol{\varphi}$ for each $n$, therefore $K_{n}^{\mathbf{c} \uparrow \boldsymbol{\pi}}$ is dense in $\mathbf{M T}(\boldsymbol{\pi})$ (then obviously open dense) and pre-dense in $\mathbf{M T}\left(\boldsymbol{\pi} \cup \cup^{\mathrm{cw}} \boldsymbol{\varphi}\right.$ ) by Lemma 10.3(i). It follows that $\left.K_{n}^{\mathbf{c} \uparrow(\boldsymbol{\pi} \cup \text { cw }} \boldsymbol{\varphi}\right)$ is dense in $\mathbf{M T}\left(\boldsymbol{\pi} \cup \cup^{\text {cw }} \boldsymbol{\varphi}\right)$.

To check (ii), (ii) apply Lemma 10.3(iv),(v).

\section{Non-principal names and avoiding refinements}

Let $\boldsymbol{\pi}$ be a multiforcing. Then $\mathbf{M T}(\boldsymbol{\pi})$ adds a collection of reals $x_{\xi k},\langle\xi, k\rangle \in|\boldsymbol{\pi}|$, where each principal real $x_{\xi k}=x_{\xi k}[G]$ is $\boldsymbol{\pi}(\xi, k)$-generic over the ground set universe. Obviously many more reals are added, and given a $\boldsymbol{\pi}$-real name $\mathbf{c}$, one can elaborate different requirements for a condition $\boldsymbol{p} \in \mathbf{M T}(\boldsymbol{\pi})$ to force that $\mathbf{c}$ is a name of a real of the form $x_{\xi k}$ or to force the opposite. But we are mostly interested in simple conditions related to the "opposite" part. The next definition provides such a condition.

Definition 16.1. Let $\boldsymbol{\pi}$ be a multiforcing, $\langle\xi, k\rangle \in|\boldsymbol{\pi}|$. A real name $\mathbf{c}$ is non-principal over $\boldsymbol{\pi}$ at $\xi, k$ if the following set is open dense in $\operatorname{MT}(\boldsymbol{\pi})$ :

$$
\boldsymbol{D}_{\xi k}^{\boldsymbol{\pi}}(\mathbf{c})=\left\{\boldsymbol{p} \in \mathbf{M T}(\boldsymbol{\pi}): \boldsymbol{p} \text { directly forces } \mathbf{c} \notin\left[T_{\xi k}^{\boldsymbol{p}}\right]\right\} .
$$

We'll show below (Theorem 18.2(i)) that the non-principality implies $\mathbf{c}$ being not a name of the real $x_{\xi k}[\underline{G}]$. And further, the avoidance condition in the next definition will be shown to imply $\mathbf{c}$ being a name of a non-generic real. 
Definition 16.2. Let $\boldsymbol{\pi}, \boldsymbol{\varphi}$ be multiforcings, $\boldsymbol{\pi} \sqsubset \boldsymbol{\varphi},\langle\xi, k\rangle \in|\boldsymbol{\pi}|$. Say that $\boldsymbol{\varphi}$ avoids a real name c over $\boldsymbol{\pi}$ at $\xi, k$, in symbol $\boldsymbol{\pi} \sqsubset_{\xi k}^{\mathbf{c}} \boldsymbol{\varphi}$, if for each $Q \in \boldsymbol{\varphi}(\xi, k)$, $\boldsymbol{\varphi}$ locks, over $\boldsymbol{\pi}$, the set

$$
\boldsymbol{D}(\mathbf{c}, Q, \boldsymbol{\pi})=\{\boldsymbol{r} \in \mathbf{M T}(\boldsymbol{\pi}): \boldsymbol{r} \text { directly forces } \mathbf{c} \notin[Q]\},
$$

in the sense of Definition 10.2 - formally $\boldsymbol{\pi} \sqsubset \boldsymbol{D ( \mathbf { c } , Q , \boldsymbol { \pi } )} \boldsymbol{Q}$.

Lemma 16.3. Assume that $\boldsymbol{\pi}, \boldsymbol{\varphi}, \boldsymbol{\sigma}$ are multiforcings, $\langle\xi, k\rangle \in|\boldsymbol{\pi}|$, and $\mathbf{c}$ is a $\boldsymbol{\pi}$-real name. Then:

(i) if $\boldsymbol{\pi} \sqsubset_{\xi k}^{\mathbf{c}} \boldsymbol{Q}$ and $Q \in \boldsymbol{Q}(\xi, k)$ then the set $\boldsymbol{D}(\mathbf{c}, Q, \boldsymbol{\pi})$ is open dense in $\mathbf{M T}(\boldsymbol{\pi})$ and pre-dense in $\mathbf{M T}\left(\boldsymbol{\pi} \cup \cup^{\mathrm{cw}} Q\right)$;

(ii) if $\boldsymbol{\pi} \sqsubset_{\xi k}^{\mathbf{c}} \boldsymbol{\varphi} \sqsubset \boldsymbol{\sigma}$ then $\boldsymbol{\pi} \sqsubset_{\xi k}^{\mathbf{c}} \boldsymbol{\sigma}$;

(iii) if $\left\langle\boldsymbol{\pi}_{\alpha}\right\rangle_{\alpha<\lambda}$ is a ᄃ-increasing sequence in $\mathbf{M F}, 0<\mu<\lambda, \boldsymbol{\pi}=\bigcup_{\alpha<\mu}^{\mathrm{cw}} \boldsymbol{\pi}_{\alpha}$, and $\boldsymbol{\pi} \sqsubset_{\xi k}^{\mathrm{c}} \boldsymbol{\pi}_{\mu}$, then $\boldsymbol{\pi} \sqsubset_{\xi k}^{\mathrm{c}} \boldsymbol{\varphi}=\bigcup_{\mu \leq \alpha<\lambda}^{\mathrm{cw}} \boldsymbol{\pi}_{\alpha}$.

Proof. (i) Apply Lemma 10.3(i).

(ii) Let $\langle\xi, k\rangle \in|\boldsymbol{\pi}|$ and $S \in \boldsymbol{\sigma}(\xi, k)$. Then, as $\boldsymbol{\varphi} \sqsubset \boldsymbol{\sigma}$, there is a finite set $\left\{Q_{1}, \ldots, Q_{m}\right\} \subseteq \boldsymbol{Q}(\xi, k)$ such that $S \subseteq Q_{1} \cup \cdots \cup Q_{m}$. We have $\boldsymbol{\pi} \sqsubset_{\boldsymbol{D}\left(\mathbf{c}, Q_{i}, \boldsymbol{\pi}\right)} \boldsymbol{\varphi}$ for all $i$ since $\boldsymbol{\pi} \sqsubset_{\xi k}^{\mathbf{c}} \boldsymbol{Q}$, therefore $\boldsymbol{\pi} \sqsubset_{\boldsymbol{D}\left(\mathbf{c}, Q_{i}, \boldsymbol{\pi}\right)} \boldsymbol{\sigma}, \forall i$, by Lemma 10.3(iv). Note that $\bigcap_{i} \boldsymbol{D}\left(\mathbf{c}, Q_{i}, \boldsymbol{\pi}\right) \subseteq \boldsymbol{D}(\mathbf{c}, S, \boldsymbol{\pi})$ since $S \subseteq \bigcup_{i} Q_{i}$. We conclude that $\boldsymbol{\pi} \sqsubset_{\boldsymbol{D}(\mathbf{c}, S, \boldsymbol{\pi})} \boldsymbol{\sigma}$ by Lemma 10.3(ii),(iii). Therefore $\boldsymbol{\pi} \sqsubset_{\xi k}^{\mathbf{c}} \boldsymbol{\sigma}$.

\section{Generic refinements avoid non-principal names}

The following theorem says that generic refinements as in Section 11 avoid nonprincipal names. It resembles Theorem 12.1 to some extent, yet the latter is not directly applicable here as both the multitree $Q$ and the set $\boldsymbol{D}(\mathbf{c}, Q, \boldsymbol{\pi})$ depend on $\boldsymbol{\varphi}$, and hence the sets $\boldsymbol{D}(\mathbf{c}, Q, \boldsymbol{\pi})$ do not necessarily belong to $\mathfrak{M}^{+}$. However the proof will be based on rather similar arguments.

Theorem 17.1. In the assumptions of Theorem 11.3, if $\langle\eta, K\rangle \in|\boldsymbol{\pi}| \subseteq \mathfrak{M}$ and $\mathbf{c} \in \mathfrak{M}$ is a $\boldsymbol{\pi}$-real name non-principal over $\boldsymbol{\pi}$ at $\eta, K$ then $\boldsymbol{\pi} \sqsubset_{\eta K}^{\mathbf{c}} \boldsymbol{Q}$.

Proof. Assume that $\boldsymbol{Q}=\boldsymbol{Q}[\rrbracket]$ is obtained from an $\mathfrak{M}^{+}$-generic sequence $₫$ of multisystems in $\mathbf{M S}(\boldsymbol{\pi})$, as in Definition 11.1. We stick to the notation of 11.1.

Let $Q \in \boldsymbol{\varphi}(\eta, K)$; we have to prove that $\boldsymbol{\varphi}$ locks the set $\boldsymbol{D}(\mathbf{c}, Q, \boldsymbol{\pi})$ over $\boldsymbol{\pi}$. By construction $Q=\boldsymbol{Q}_{\eta K, \widetilde{m}}^{\bowtie}\left(s_{0}\right) \subseteq \boldsymbol{Q}_{\eta K, \widetilde{m}}^{\bowtie}$ for some $\widetilde{m}<\omega$; it can be assumed that $Q=\boldsymbol{Q}_{\eta K, \widetilde{m}}^{\llbracket}$. Following the proof of Theorem 12.1, we suppose that $\boldsymbol{p} \in \mathbf{M T}(\boldsymbol{\pi})$, $\boldsymbol{u} \in \mathbf{M T}(\boldsymbol{\varphi}),|\boldsymbol{u}| \cap|\boldsymbol{p}|=\varnothing$, define $X=|\boldsymbol{u}|, Y=|\boldsymbol{\pi}| \backslash X$, and assume that $T_{\xi k}^{u}=\boldsymbol{Q}_{\xi k, m_{\xi k}}^{\natural}$, where $m_{\xi k}<\omega$, for each $\langle\xi, k\rangle \in X$.

Consider the set $\Delta$ of all multisystems $\varphi \in \operatorname{MS}(\pi)$ such that there is a number $H>0$ and a multitree $\boldsymbol{q} \in \mathbf{M S}(\boldsymbol{\pi})$ satisfying conditions 
(1) $|\boldsymbol{q}| \cap X=\varnothing$ and $\boldsymbol{q} \leqslant \boldsymbol{p}$;

(2) if $\langle\xi, k\rangle \in X$ then $\left\langle\xi, k, m_{\xi k}\right\rangle \in|\varphi|$;

(3) if $\langle\xi, k, m\rangle \in|\varphi|$ then $h^{\varphi}(\xi, k, m)=H$;

(but not (4) though) as in the proof of Theorem 12.1, along with two more requirements

(5) $\langle\eta, K, \widetilde{m}\rangle \in|\varphi|$ - hence still $h^{\varphi}(\eta, K, \widetilde{m})=H$ by $(3)$;

(6) if $s \in 2^{H}$ and $\tau \in\left(2^{H}\right)^{X}$ then $\mathbf{s}(\boldsymbol{\varphi}, \tau) \cup \boldsymbol{q}$ directly forces $\mathbf{c} \notin\left[T_{\eta K, \widetilde{m}}^{\varphi}(s)\right]$.

Lemma 17.2. $\Delta$ is dense in $\operatorname{MS}(\boldsymbol{\pi})$.

Proof. Suppose that $\boldsymbol{\psi} \in \mathbf{M S}(\boldsymbol{\pi})$; we can assume that $\boldsymbol{\psi}$ already satisfies

(a) if $\langle\xi, k\rangle \in X$ then $\left\langle\xi, k, m_{\xi k}\right\rangle \in|\boldsymbol{\psi}|$;

(b) there is a number $g<\omega$ such that $h^{\psi}(\xi, k, m)=g$ for all $\langle\xi, k, m\rangle \in|\boldsymbol{\psi}|$;

as in Lemma 12.2, and in addition $\langle\eta, K, \widetilde{m}\rangle \in|\boldsymbol{\psi}|$.

Let $H=g+1$. Define a multisystem $\chi \in \mathbf{M S}(\boldsymbol{\pi})$ so that $|\chi|=|\boldsymbol{\psi}|$, and $h^{\chi}(\xi, k, m)=H, T_{\xi k, m}^{\chi}\left(s^{\frown} i\right)=T_{\xi k, m}^{\psi}(s)(\rightarrow i)$ for all $\langle\xi, k, m\rangle \in|\boldsymbol{\psi}|$ and $s^{\curvearrowleft} i \in 2^{H}$; then $\boldsymbol{\psi} \preccurlyeq \chi$. We claim that there is a multitree $\boldsymbol{q} \in \mathbf{M T}(\boldsymbol{\pi})$ satisfying (1), and a multisystem $\boldsymbol{\varphi} \in \mathbf{M S}(\boldsymbol{\pi})$ satisfying (6) and such that still $|\boldsymbol{\varphi}|=|\boldsymbol{\psi}|$ and $h^{\varphi}(\xi, k, m)=H$ for all $\langle\xi, k, m\rangle \in|\boldsymbol{\psi}|$, and in addition

(c) if $\langle\xi, k\rangle \in X$ and $s \in 2^{H}$ then $T_{\xi k, m_{\xi k}}^{\varphi}(s) \subseteq T_{\xi k, m_{\xi k}}^{\chi}(s)$, and we also have $T_{\eta K, \widetilde{m}}^{\varphi}(s) \subseteq T_{\eta K, \widetilde{m}}^{\chi}(s) ;$

(d) $T_{\xi k, m}^{\varphi}(s)=T_{\xi k, m}^{\chi}(s)$ for all applicable $\xi, k, m, s$ not covered by (c).

To achieve (6) in one step for one particular $\tau \in\left(2^{H}\right)^{X}$, consider the multitree $\boldsymbol{r}=\mathbf{s}(\boldsymbol{\chi}, \tau) \cup \boldsymbol{p}$. By Lemma 14.1 and the density assumption of the theorem, there is a multitree $\boldsymbol{r}^{\prime} \in \mathbf{M T}(\boldsymbol{\varphi}), \boldsymbol{r}^{\prime} \leqslant \boldsymbol{r}$, which directly forces $\mathbf{c} \notin\left[T_{\eta K}^{\boldsymbol{r}^{\prime}}\right]$, and there are multitrees $U_{s} \in \mathbf{M T}(\boldsymbol{\varphi}), s \in 2^{H}$, such that $U_{s} \subseteq T_{\eta K, \widetilde{m}}^{\chi}(s)$ and $\boldsymbol{r}^{\prime}$ directly forces $\mathbf{c} \notin\left[U_{s}\right], \forall s$. Let $\chi^{\prime}$ be obtained from $\chi$ by the following reassignment.

(I) We set $T_{\xi k, m_{\xi k}}^{\chi^{\prime}}(\tau(\xi, k))=T_{\xi k}^{r^{\prime}}$ for all $\langle\xi, k\rangle \in X$.

(II) If $s \in 2^{H}$, and either $\langle\eta, K\rangle \notin X$, or $\widetilde{m} \neq m_{\eta K}$, or $s \neq \tau(\eta, K)$ then we set $T_{\eta K, \widetilde{m}}^{\chi^{\prime}}(s)=U_{s}$. (Note that if $\langle\eta, K\rangle \in X$ and $\widetilde{m}=m_{\eta K}$ then the tree $T_{\eta K, \tilde{m}}^{\chi^{\prime}}(\tau(\eta, K))=T_{\eta K}^{\boldsymbol{r}^{\prime}}$ is already defined by (I).) 
Let $\boldsymbol{p}^{\prime}=\boldsymbol{r}^{\prime} \uparrow Y$, so that $\boldsymbol{r}^{\prime}=\mathbf{s}\left(\chi^{\prime}, \tau\right) \cup \boldsymbol{p}^{\prime}$. By construction the tree $\boldsymbol{p}^{\prime}$ satisfies (6), for the system $\tau$ chosen, in the case $\langle\eta, K\rangle \in X, \widetilde{m}=m_{\eta K}, s=\tau(\eta, K)$ by

(I) and in all other cases by (II).

Now consider another $\tau^{\prime} \in\left(2^{H}\right)^{X}$ and the multitree $\boldsymbol{r}^{\prime}=\mathbf{s}\left(\boldsymbol{\chi}^{\prime}, \tau^{\prime}\right) \cup \boldsymbol{p}^{\prime}$. There is a multitree $\boldsymbol{r}^{\prime \prime} \in \mathbf{M T}(\boldsymbol{\pi}), \boldsymbol{r}^{\prime \prime} \leqslant \boldsymbol{r}^{\prime}$, which which directly forces $\mathbf{c} \notin\left[T_{\eta K}^{\boldsymbol{r}^{\prime}}\right]$ and $\mathbf{c} \notin\left[U_{s}^{\prime}\right]$ for each $s \in 2^{H}$, where $U_{s}^{\prime} \in \mathbf{M T}(\boldsymbol{\varphi})$ and $U_{s}^{\prime} \subseteq T_{\eta K, \widetilde{m}}^{\chi^{\prime}}(s)$. Let $\chi^{\prime \prime}$ be obtained from $\chi^{\prime}$ by the the same reassignment (for $\tau^{\prime}$ instead of $\tau$ ).

And so on. The final multisystem and multitree of this construction will be $\boldsymbol{\varphi}$ and $\boldsymbol{q}$ satisfying (1), (2), (3), (5), (6).

$\square$ (Lemma)

Come back to the theorem. Note that $\Delta \in \mathfrak{M}^{+}$, similarly to the proof of Theorem 12.1. Therefore, by the lemma, there is an index $j$ such that the system $\varphi(j)$ belongs to $\Delta$. Let this be witnessed by a number $H>0$ and a multitree $\boldsymbol{q} \in \mathbf{M T}(\boldsymbol{\pi})$, such that conditions (1), (2), (3), (5), (6) are satisfied for $\varphi=\varphi(j)$.

It remains to prove that $\boldsymbol{u} \subseteq{ }^{\text {fin }} \bigvee \boldsymbol{D}(\mathbf{c}, Q, \boldsymbol{\pi})_{\boldsymbol{q}}^{|\boldsymbol{u}|}$. Let $V$ consist of all multitrees $\boldsymbol{v}=\mathbf{s}(\boldsymbol{\varphi}(j), \tau)$, where $\tau \in\left(2^{H}\right)^{X} ;[\boldsymbol{u}] \subseteq \bigcup_{\boldsymbol{v} \in V}[\boldsymbol{v}]$ by construction.

Further, if $s \in 2^{H}$ and $\boldsymbol{v} \in V$ then $\boldsymbol{v} \cup \boldsymbol{q}$ directly forces $\mathbf{c} \notin\left[T_{\eta K, \widetilde{m}}^{\varphi(j)}(s)\right]$ by (6), that is, directly forces $\mathbf{c} \notin\left[\boldsymbol{T}_{\eta K, \widetilde{m}}^{\bowtie}(s)\right]$ in the notation of Definition 11.1. Therefore $\boldsymbol{v} \cup \boldsymbol{q}$ directly forces $\mathbf{c} \notin\left[\boldsymbol{Q}_{\eta K, \widetilde{m}}^{\natural}(s)\right]$ since $\boldsymbol{Q}_{\eta K, \widetilde{m}}^{\triangleleft}(s) \subseteq \boldsymbol{T}_{\eta K, \widetilde{m}}^{\bowtie}(s)$ by Lemma 11.3(b). However $Q=\boldsymbol{Q}_{\eta K, \widetilde{m}}^{\bowtie /}=\bigcup_{s \in 2^{H}} \boldsymbol{Q}_{\eta K, \widetilde{m}}^{\llbracket p}(s)$ by Lemma 11.3(a). It follows that $\boldsymbol{v} \cup \boldsymbol{q}$ directly forces $\mathbf{c} \notin[Q]$, that is, $\boldsymbol{v} \in \boldsymbol{D}(\mathbf{c}, Q, \boldsymbol{\pi})_{\boldsymbol{q}}^{|\boldsymbol{u}|}$.

We conclude that $V$ is a (finite) subset of $\boldsymbol{D}(\mathbf{c}, Q, \boldsymbol{\pi})_{\boldsymbol{q}}^{|\boldsymbol{u}|}$. And this accomplishes the proof of $\boldsymbol{u} \subseteq$ fin $\bigvee \boldsymbol{D}(\mathbf{c}, Q, \boldsymbol{\pi})_{\boldsymbol{q}}^{|\boldsymbol{u}|}$.

\section{Consequences for reals in generic extensions}

We first prove a result saying that all reals in $\mathbf{M T}(\boldsymbol{\pi})$-generic extensions are adequately represented by real names. Then Theorem 18.2 will show effects of the property of being a non-principal name.

Proposition 18.1. Suppose that $\boldsymbol{\pi}$ is a regular multiforcing, $G \subseteq \mathbf{M T}(\boldsymbol{\pi})$ is generic over the ground set universe $\mathbf{V}$, and $x \in \mathbf{V}[G] \cap \omega^{\omega}$. Then

(i) there is a true $\boldsymbol{\pi}$-real name $\mathbf{c} \in \mathbf{V}$ such that $x=\mathbf{c}[G]$;

(ii) if $\mathbf{M T}(\boldsymbol{\pi})$ is a CCC forcing in $\mathbf{V}$ then there is a small true $\boldsymbol{\pi}$-real name $\mathbf{d} \in \mathbf{V}$ with $x=\mathbf{d}[G]$.

Proof. (i) is an instances of a general forcing theorem (see Remark 13.5 on the effect of regularity). To prove (ii), pick a real name $\mathbf{c}$ by (i), extend each set 
$K_{n}^{\mathbf{c}}=\bigcup_{i} K_{n i}^{\mathbf{c}}$ to an open dense set $O_{n}$ by closing strongwards, choose maximal antichains $A_{n} \subseteq O_{n}$ in those sets - which have to be countable by CCC, and then let $A_{n i}=A_{n} \cap K_{n i}^{\mathbf{c}}$ and $\mathbf{d}=\left\{\langle\boldsymbol{p}, n, i\rangle: \boldsymbol{p} \in A_{n i}\right\}$.

Theorem 18.2. Let $\boldsymbol{\pi}$ be a regular multiforcing. Then

(i) if a set $G \subseteq \mathbf{M T}(\boldsymbol{\pi})$ is generic over the ground set universe $\mathbf{V},\langle\xi, k\rangle \in|\boldsymbol{\pi}|$, and $x \in \mathbf{V}[G] \cap \omega^{\omega}$, then $x \neq x_{\xi k}[G]$ if and only if there is a true $\boldsymbol{\pi}$-real name $\mathbf{c}$, non-principal over $\boldsymbol{\pi}$ at $\xi, k$ and such that $x=\mathbf{c}[G]$.

(ii) if $\mathbf{c}$ is a $\boldsymbol{\pi}$-real name, $\langle\xi, k\rangle \in|\boldsymbol{\pi}|, \boldsymbol{\varphi}$ is a multiforcing, $\boldsymbol{\pi} \sqsubset_{\xi k}^{\mathbf{c}} \boldsymbol{Q}$, and a set $G \subseteq \mathbf{M T}\left(\boldsymbol{\pi} \cup^{\mathrm{cw}} \boldsymbol{\varphi}\right)$ is generic over $\mathbf{V}$ then $\mathbf{c}[G] \notin \bigcup_{Q \in \boldsymbol{Q}(\xi, k)}[Q]$.

Proof. (i) Suppose that $x \neq x_{\xi k}[G]$. By a known forcing theorem, there is a true $\boldsymbol{\pi}$-real name $\mathbf{c}$ such that $x=\mathbf{c}[G]$ and $\mathbf{M T}(\boldsymbol{\pi})$ forces that $\mathbf{c} \neq x_{\xi k}[\underline{G}]$. It remains to show that $\mathbf{c}$ is a non-principal name over $\boldsymbol{\pi}$ at $\xi, k$. We have to prove that the set

$$
\boldsymbol{D}_{\xi k}^{\boldsymbol{\pi}}(\mathbf{c})=\left\{\boldsymbol{p} \in \mathbf{M T}(\boldsymbol{\pi}): \boldsymbol{p} \text { directly forces } \mathbf{c} \notin\left[T_{\xi k}^{\boldsymbol{p}}\right]\right\} .
$$

is open dense in $\mathbf{M T}(\boldsymbol{\pi})$. The openness is clear, let us prove the density. Consider an arbitrary $\boldsymbol{q} \in \mathbf{M T}(\boldsymbol{\pi})$. Then $\boldsymbol{q} \mathbf{M T}(\boldsymbol{\pi})$-forces $\mathbf{c} \neq x_{\xi k}[\underline{G}]$ by the choice of $\mathbf{c}$, hence we can assume that, for some $n$, it is $\mathbf{M T}(\boldsymbol{\pi})$-forced by $\boldsymbol{q}$ that $\mathbf{c}(n) \neq x_{\xi k}[\underline{G}](n)$. Then by Lemma 14.1(i) there is a multitree $\boldsymbol{p} \in \mathbf{M T}(\boldsymbol{\pi})$, $\boldsymbol{p} \leqslant \boldsymbol{q}$, and a string $s \in \omega^{n+1}$, such that $\boldsymbol{p} \mathbf{M T}(\boldsymbol{\pi})$-forces $s \subseteq \mathbf{c}$. Now it suffices to show that $s \notin T_{\xi k}^{p}$. Suppose otherwise: $s \in T_{\xi k}^{p}$. Then the tree $T=T_{\xi k}^{p} \uparrow_{s}$ still belongs to $\mathbf{M T}(\boldsymbol{\pi})$. Therefore the multitree $\boldsymbol{r}$ defined by $T_{\xi k}^{r}=T$ and $T_{\xi^{\prime} k^{\prime}}^{r}=T_{\xi^{\prime} k^{\prime}}^{p}$ for each pair $\left\langle\xi^{\prime}, k^{\prime}\right\rangle \neq\langle\xi, k\rangle$, belongs to $\mathbf{M T}(\boldsymbol{\pi})$ and satisfies $\boldsymbol{r} \leqslant \boldsymbol{p} \leqslant \boldsymbol{q}$. However $\boldsymbol{r}$ directly forces both $\mathbf{c}(n)$ and $x_{\xi k}[\underline{G}](n)$ to be equal to one and the same value $\ell=s(n)$, which contradicts to the choice of $n$.

To prove the converse let $\mathbf{c} \in \mathbf{V}$ be a real name non-principal over $\boldsymbol{\pi}$ at $\xi, k$, and $x=\mathbf{c}[G]$. Assume to the contrary that $\langle\xi, k\rangle \in|\boldsymbol{\pi}|$ and $x=x_{\xi k}[G]$. There is a multitree $\boldsymbol{q} \in G$ which $\mathbf{M T}(\boldsymbol{\pi})$-forces $\mathbf{c}=x_{\xi k}[\underline{G}]$. As $\mathbf{c}$ is non-principal, there is a stronger multitree $\boldsymbol{p} \in G \cap \boldsymbol{D}_{\xi k}^{\boldsymbol{\pi}}(\mathbf{c}), \boldsymbol{p} \leqslant \boldsymbol{q}$. Thus $\boldsymbol{p}$ directly forces $\mathbf{c} \notin\left[T_{\xi k}^{\boldsymbol{p}}\right]$, and hence $\mathbf{M T}(\boldsymbol{\pi})$-forces the same statement. Yet $\boldsymbol{p} \mathbf{M T}(\boldsymbol{\pi})$-forces $\dot{\boldsymbol{x}}_{\xi k} \in\left[T_{\xi k}^{\boldsymbol{p}}\right]$, of course, and this is a contradiction.

(ii) Suppose towards the contrary that $Q \in \varphi(\xi, k)$ and $\mathbf{c}[G] \in[Q]$. By definition, $\boldsymbol{\varphi}$ locks, over $\boldsymbol{\pi}$, the set

$$
\boldsymbol{D}(\mathbf{c}, Q, \boldsymbol{\pi})=\{\boldsymbol{r} \in \mathbf{M T}(\boldsymbol{\pi}): \boldsymbol{r} \text { directly forces } \mathbf{c} \notin[Q]\} .
$$

Therefore in particular $\boldsymbol{D}(\mathbf{c}, Q, \boldsymbol{\pi})$ is pre-dense in $\mathbf{M T}\left(\boldsymbol{\pi} \cup^{\mathrm{cw}} \boldsymbol{\varphi}\right)$ by Lemma 10.3. We conclude that $G \cap \boldsymbol{D}(\mathbf{c}, Q, \boldsymbol{\pi}) \neq \varnothing$. In other words, there is a multitree $\boldsymbol{r} \in \mathbf{M T}(\boldsymbol{\pi})$ which directly forces $\mathbf{c} \notin[Q]$. It easily follows that $\mathbf{c}[G] \notin[Q]$, which is a contradiction. 


\section{Combining refinement types}

Here we summarize the properties of generic refinements considered above. The next definition combines the refinement types $\sqsubset_{D}, \sqsubset_{\boldsymbol{D}}, \sqsubset_{\xi k}^{\mathbf{c}}$.

Definition 19.1. Suppose that $\pi \sqsubset \boldsymbol{Q}$ are multiforcings and $\mathfrak{M} \in \mathrm{HC}$ is any set. Let $\boldsymbol{\pi} \mathbb{L}_{\mathfrak{M}} \boldsymbol{\varphi}$ mean that the four following requirements hold:

(1) if $\langle\xi, k\rangle \in|\boldsymbol{\pi}|, D \in \mathfrak{M}, D \subseteq \boldsymbol{\pi}(\xi, k), D$ is pre-dense in $\boldsymbol{\pi}(\xi, k)$, then $\boldsymbol{\pi}(\xi, k) \sqsubset{ }_{D} \boldsymbol{Q}(\xi, k)$;

(2) if $\boldsymbol{D} \in \mathfrak{M}, \boldsymbol{D} \subseteq \mathbf{M T}(\boldsymbol{\pi}), \boldsymbol{D}$ is open dense in $\mathbf{M T}(\boldsymbol{\pi})$, then $\boldsymbol{\pi} \sqsubset \boldsymbol{D} \boldsymbol{\boldsymbol { q }}$;

(3) if $\mathbf{c} \in \mathfrak{M}$ is a $\boldsymbol{\pi}$-real name then $\boldsymbol{\pi} \sqsubset_{\mathbf{c}} \boldsymbol{\varphi}$;

(4) if $\langle\xi, k\rangle \in|\boldsymbol{\pi}|$ and $\mathbf{c} \in \mathfrak{M}$ is a $\boldsymbol{\pi}$-real name, non-principal over $\boldsymbol{\pi}$ at $\xi, k$, then $\pi \sqsubset_{\xi k}^{\mathrm{c}}$ Q.

Corollary 19.2 (of lemmas $9.4,10.3,15.3,16.3$ ). Let $\boldsymbol{\pi}, \boldsymbol{\varphi}, \boldsymbol{\sigma}$ be multiforcings and $\mathfrak{M}$ be a countable set. Then:

(i) if $\boldsymbol{\pi} \square_{\mathfrak{M}} \boldsymbol{\varphi} \sqsubset \boldsymbol{\sigma}$ then $\boldsymbol{\pi} \square_{\mathfrak{M}} \boldsymbol{\sigma}$;

(ii) if $\left\langle\boldsymbol{\pi}_{\alpha}\right\rangle_{\alpha<\lambda}$ is a ᄃ-increasing sequence in $\mathbf{M F}, 0<\mu<\lambda, \boldsymbol{\pi}=\bigcup_{\alpha<\mu}^{\mathrm{cw}} \boldsymbol{\pi}_{\alpha}$, and $\boldsymbol{\pi} \square_{\mathfrak{M}} \boldsymbol{\pi}_{\mu}$, then $\boldsymbol{\pi} \llbracket_{\mathfrak{M}} \boldsymbol{\varphi}=\bigcup_{\mu \leq \alpha<\lambda}^{\mathrm{cw}} \boldsymbol{\pi}_{\alpha}$.

Corollary 19.3. If $\boldsymbol{\pi}$ is a small multiforcing, $\mathfrak{M} \in \mathrm{HC}$, and $\boldsymbol{\varphi}$ is an $\mathfrak{M}$-generic refinement of $\boldsymbol{\pi}$ (exists by Proposition 11.2!), then $\boldsymbol{\pi} \mathbb{L}_{\mathfrak{M}} \boldsymbol{\varphi}$.

Proof. We have $\boldsymbol{\pi} \llbracket_{\mathfrak{M}} \boldsymbol{\varphi}$ by a combination of 11.3(ii), 12.1, 15.2, and 17.1. 


\section{The forcing notion}

In this chapter we define the forcing notion to prove the main theorem. It will have the form $\mathbf{M T}(\square)$, for a certain multiforcing $\llbracket$ with $|\llbracket|=\omega_{1} \times \omega$. The multiforcing $\llbracket$ will be equal to the componentwise union of terms of a certain increasing sequence $\vec{\mathbb{}}$ of small multiforcings. And quite a complicated construction of this sequence in $\mathbf{L}$ will make use of some ideas related to diamond-style constructions, as well as to some sort of definable genericity.

\section{Increasing sequences of small multiforcings}

Recall that $\mathbf{M F}$ is the set of all multiforcings (Section 7). Let $\mathbf{s M F} \subseteq \mathbf{M F}$ be the set of all small special multiforcings; s accounts for both small and special. Thus a multiforcing $\boldsymbol{\pi} \in \mathbf{M F}$ belongs to sMF if $|\boldsymbol{\pi}|$ is (at most) countable and if $\langle\xi, k\rangle \in|\boldsymbol{\pi}|$ then $\boldsymbol{\pi}(\xi, k)$ is a small special (Definition 5.4) forcing in PTF.

Definition 20.1. Let $\overrightarrow{\mathbf{s M F}}$, resp., $\overrightarrow{\mathbf{s M F}}_{\omega_{1}}$ be the set of all $\sqsubset$-increasing sequences $\overrightarrow{\boldsymbol{\pi}}=\left\langle\boldsymbol{\pi}_{\alpha}\right\rangle_{\alpha<\kappa}$ of multiforcings $\boldsymbol{\pi}_{\alpha} \in \mathbf{s M F}$, of length $\kappa=\operatorname{dom}(\overrightarrow{\boldsymbol{\pi}})<\omega_{1}$, resp., $\kappa=\omega_{1}$, which are domain-continuous, in the sense that if $\lambda<\kappa$ is a limit ordinal then $\left|\boldsymbol{\pi}_{\lambda}\right|=\bigcup_{\alpha<\lambda}\left|\boldsymbol{\pi}_{\alpha}\right|$. Sequences in $\overrightarrow{\mathbf{s M F}} \cup \overrightarrow{\mathbf{s M F}}_{\omega_{1}}$ are called multisequences. We order $\overrightarrow{\mathbf{s M F}} \cup \mathbf{S M F} \omega_{\omega_{1}}$ by the usual relations $\subseteq$ and $\subset$ of extension of sequences.

- Thus $\overrightarrow{\boldsymbol{\pi}} \subset \overrightarrow{\boldsymbol{\varphi}}$ iff $\kappa=\operatorname{dom}(\overrightarrow{\boldsymbol{\pi}})<\lambda=\operatorname{dom}(\overrightarrow{\boldsymbol{\varphi}})$ and $\boldsymbol{\pi}_{\alpha}=\boldsymbol{\varphi}_{\alpha}$ for all $\alpha<\kappa$.

- In this case, if $\mathfrak{M}$ is any set, and $\boldsymbol{\varphi}_{\kappa}$ (the first term of $\overrightarrow{\boldsymbol{\varphi}}$ absent in $\overrightarrow{\boldsymbol{\pi}}$ ) satisfies $\boldsymbol{\pi} \sqsubset_{\mathfrak{M}} \boldsymbol{\varphi}_{\kappa}$, where $\boldsymbol{\pi}=\bigcup_{\alpha<\kappa}^{\mathrm{cw}} \boldsymbol{\pi}_{\alpha}$, then we write $\overrightarrow{\boldsymbol{\pi}} \subset_{\mathfrak{M}} \overrightarrow{\boldsymbol{\varphi}}$.

If $\overrightarrow{\boldsymbol{\pi}}$ is a multisequence in $\overrightarrow{\mathbf{s M F}} \cup \overrightarrow{\mathbf{s M F}}_{\omega_{1}}$ then let $\mathbf{M T}(\overrightarrow{\boldsymbol{\pi}})=\mathbf{M T}(\boldsymbol{\pi})$, where $\boldsymbol{\pi}=\bigcup^{\mathrm{cw}} \overrightarrow{\boldsymbol{\pi}}=\bigcup_{\alpha<\kappa}^{\mathrm{cw}} \boldsymbol{\pi}_{\alpha}$ (componentwise union), and $\kappa=$ dom $\overrightarrow{\boldsymbol{\pi}}$. Accordingly a (true) $\overrightarrow{\boldsymbol{\pi}}$-real name will mean a (true) $\boldsymbol{\pi}$-real name.

Corollary 20.2. Suppose that $\kappa<\lambda<\omega_{1}, \mathfrak{M}$ is a countable set, and $\overrightarrow{\boldsymbol{\pi}}=$ $\left\langle\boldsymbol{\pi}_{\alpha}\right\rangle_{\alpha<\kappa}$ is a multisequence in $\overrightarrow{\mathbf{s M F}}$. Then:

(i) the componentwise union $\boldsymbol{\pi}=\bigcup^{\mathrm{cw}} \overrightarrow{\boldsymbol{\pi}}=\bigcup_{\alpha<\kappa}^{\mathrm{cw}} \boldsymbol{\pi}_{\alpha}$ is a regular multiforcing;

(ii) there is a multisequence $\overrightarrow{\boldsymbol{\varphi}} \in \overrightarrow{\mathbf{s M F}}$ satisfying $\operatorname{dom}(\overrightarrow{\boldsymbol{\varphi}})=\lambda$ and $\overrightarrow{\boldsymbol{\pi}} \subset_{\mathfrak{M}} \overrightarrow{\boldsymbol{\varphi}}$;

(iii) if moreover $\left\langle s_{\alpha}\right\rangle_{\alpha<\lambda}$ is an $\subset$-increasing sequence of countable sets $s_{\alpha} \subseteq$ $\omega_{1} \times \omega, s_{\alpha}=\left|\pi_{\alpha}\right|$ for all $\alpha<\kappa$, and $s_{\gamma}=\bigcup_{\alpha<\gamma} s_{\alpha}$ for all limit $\gamma<\lambda$, then there is a multisequence $\overrightarrow{\boldsymbol{\varphi}} \in \overrightarrow{\mathbf{s M F}}$ satisfying $\operatorname{dom}(\overrightarrow{\boldsymbol{\varphi}})=\lambda,\left|\boldsymbol{\varphi}_{\alpha}\right|=s_{\alpha}$ for all $\alpha<\lambda$, and $\overrightarrow{\boldsymbol{\pi}} \subset_{\mathfrak{M}} \overrightarrow{\boldsymbol{\varphi}}$

(iv) if $\overrightarrow{\boldsymbol{\pi}}, \overrightarrow{\boldsymbol{\rho}}, \overrightarrow{\boldsymbol{\rho}} \in \overrightarrow{\mathbf{s M F}}$ and $\overrightarrow{\boldsymbol{\pi}} \subset_{\mathfrak{M}} \overrightarrow{\boldsymbol{\rho}} \subseteq \overrightarrow{\boldsymbol{\rho}}$ then $\overrightarrow{\boldsymbol{\pi}} \subset_{\mathfrak{M}} \overrightarrow{\boldsymbol{\rho}}$; 
(v) if $\overrightarrow{\boldsymbol{\varphi}}=\left\langle\boldsymbol{\varphi}_{\alpha}\right\rangle_{\alpha<\lambda} \in \overrightarrow{\mathbf{s M F}}$ and $\overrightarrow{\boldsymbol{\pi}} \subset_{\mathfrak{M}} \overrightarrow{\boldsymbol{\varphi}}$ then $\boldsymbol{\pi}=\bigcup_{\alpha<\kappa}^{\mathrm{cw}} \boldsymbol{\pi}_{\alpha} \square_{\mathfrak{M}} \boldsymbol{\rho}_{\beta}$ whenever $\lambda \leq \beta<\mu$, and also $\pi \mathbb{L}_{\mathfrak{M}} \boldsymbol{\varphi}^{\prime}=\bigcup_{\lambda \leq \beta<\mu}^{\mathrm{CW}} \boldsymbol{\phi}_{\beta}$, therefore

(a) $\mathbf{M T}\left(\boldsymbol{\varphi}^{\prime}\right)$ is open dense in $\mathbf{M T}(\overrightarrow{\boldsymbol{\varphi}})$,

(b) if $\langle\xi, k\rangle \in|\boldsymbol{\pi}|, D \in \mathfrak{M}, D \subseteq \boldsymbol{\pi}(\xi, k), D$ is pre-dense in $\boldsymbol{\pi}(\xi, k)$, then $D$ remains pre-dense in $\boldsymbol{\pi}(\xi, k) \cup \boldsymbol{\varphi}(\xi, k)$,

(c) if $\boldsymbol{D} \in \mathfrak{M}, \boldsymbol{D} \subseteq \mathbf{M T}(\overrightarrow{\boldsymbol{\pi}}), \boldsymbol{D}$ is open dense in $\mathbf{M T}(\overrightarrow{\boldsymbol{\pi}})$, then $\boldsymbol{D}$ is pre-dense in $\mathbf{M T}\left(\boldsymbol{\pi} \cup^{\mathrm{cw}} \boldsymbol{\varphi}^{\prime}\right)=\mathbf{M T}(\overrightarrow{\boldsymbol{\varphi}})$.

Proof. (i) Make use of Lemma 9.2(iv).

(ii) We define terms $\boldsymbol{\varphi}_{\alpha}$ of the multisequence $\boldsymbol{Q}$ required by induction.

Naturally put $\boldsymbol{\varphi}_{\alpha}=\boldsymbol{\pi}_{\alpha}$ for each $\alpha<\kappa$.

Now suppose that $\kappa \leq \gamma<\lambda$, multiforcings $\boldsymbol{\varphi}_{\alpha}, \alpha<\gamma$, are defined, and $\overrightarrow{\boldsymbol{\rho}}=\left\langle\boldsymbol{Q}_{\alpha}\right\rangle_{\alpha<\gamma}$ is a multisequence in $\overrightarrow{\mathbf{s M F}}$. To define $\boldsymbol{\varphi}_{\gamma}$, assume first that $\gamma$ is limit. Let $\boldsymbol{\rho}=\bigcup^{\mathrm{cw}} \overrightarrow{\boldsymbol{\rho}}=\bigcup_{\alpha<\gamma}^{\mathrm{cw}} \boldsymbol{\varphi}_{\alpha}$ (componentwise union). We can assume that $\mathfrak{M}$ contains $\vec{\rho}$ and satisfies $\gamma \subseteq \mathfrak{M}$ (otherwise take a bigger set). By Proposition 11.2, there is an $\mathfrak{M}$-generic refinement $\boldsymbol{\varphi}$ of $\boldsymbol{\rho}$. By Theorem 11.3, $\boldsymbol{Q}$ is small special multiforcing, $\boldsymbol{\rho} \sqsubset \boldsymbol{\varphi}$, and $\boldsymbol{\rho}_{\alpha} \sqsubset \boldsymbol{\varphi}$ for all $\alpha<\gamma$. In addition $\boldsymbol{\rho} \mathbb{E}_{\mathfrak{M}} \boldsymbol{\rho}$ by Corollary 19.3. We let $\boldsymbol{\rho}_{\gamma}=\boldsymbol{\rho}$. The extended multisequence $\overrightarrow{\boldsymbol{\rho}}_{+}=\left\langle\boldsymbol{\rho}_{\alpha}\right\rangle_{\alpha<\gamma+1}$ belongs to $\overrightarrow{\mathbf{s M F}}$ and satisfies $\overrightarrow{\boldsymbol{\rho}} \subset_{\mathfrak{M}} \overrightarrow{\boldsymbol{\rho}}_{+}$.

(iii) The proof is similar, with the extra care of $\left|\boldsymbol{\varphi}_{\alpha}\right|=s_{\alpha}$.

To prove the main claim of (v) make use of Corollary 19.2.

(iv) The relation $\overrightarrow{\boldsymbol{\pi}} \subset_{\mathfrak{M}} \overrightarrow{\boldsymbol{\varphi}}$ involves only the first term of $\overrightarrow{\boldsymbol{\varphi}}$ absent in $\overrightarrow{\boldsymbol{\pi}}$.

To prove (v)(a) apply Corollary 10.1 .

(v)(b) As $\boldsymbol{\pi} \sqsubset_{\mathfrak{M}} \boldsymbol{Q}^{\prime}$ and $D \in \mathfrak{M}$, we have $\boldsymbol{\pi}(\xi, k) \sqsubset{ }_{D} \boldsymbol{\varphi}(\xi, k)$. Therefore $D$ is pre-dense in $\boldsymbol{Q}(\xi, k)$ by Lemma 9.4(ii).

(v)(c) Similarly $\boldsymbol{\pi} \sqsubset{ }_{\boldsymbol{D}} \boldsymbol{\varphi}^{\prime}, \boldsymbol{D}$ is pre-dense in $\mathbf{M T}(\overrightarrow{\boldsymbol{\varphi}})$ by Lemma 10.3(i).

Our plan regarding the forcing notion for Theorem 1.1 will be to define a certain multisequence $\overrightarrow{\mathbb{\Pi}}$ in $\overrightarrow{\mathbf{s M F}}_{\omega_{1}}$ and the ensuing multiforcing $\square=\bigcup^{\mathrm{cw}} \overrightarrow{\mathbb{T}}$ with remarkable properties related to definability and its own genericity of some sort. But we need first to introduce an important notion involved in the construction.

\section{Layer restrictions of multiforcings and deciding sets}

The construction of the mentioned multiforcing $\square$ will be maintained in such a way that different layers $\langle\llbracket(k, \xi)\rangle_{\xi<\omega_{1}}, k<\omega$, appear rather independent of each other, albeit the principal inductive parameter will be $\xi$ rather than $k$. To reflect this feature, we introduce here a suitable notation related to layer restrictions. If 
$m<\omega$ then, using a special "layer restriction" symbol $\|$ to provide a transparent distinction from the ordinary restriction $\uparrow$, we define sets of multitrees:

$$
\begin{aligned}
& \text { MT } \pi_{<m}=\text { all multitrees } \boldsymbol{p} \in \text { MT such that }|\boldsymbol{p}| \subseteq \omega_{1} \times m, \\
& \text { MT } \pi_{\geq m}=\text { all multitrees } \boldsymbol{p} \in \mathbf{M T} \text { with }|\boldsymbol{p}| \subseteq \omega_{1} \times(\omega \backslash m), \\
& \text { MT } \Uparrow_{m}=\text { all multitrees } \boldsymbol{p} \in \mathbf{M T} \text { such that }|\boldsymbol{p}| \subseteq \omega_{1} \times\{m\},
\end{aligned}
$$

and, given a multiforcing $\boldsymbol{\pi}$, define $\mathbf{M T}(\boldsymbol{\pi})\left\|_{<m}, \mathbf{M T}(\boldsymbol{\pi})\right\|_{\geq m}, \mathbf{M T}(\boldsymbol{\pi}) \|_{m}$ similarly. Accordingly if $\boldsymbol{p} \in \mathbf{M T}$ then define the layer restriction $\boldsymbol{p}\left\|_{<m} \in \mathbf{M T}\right\|_{<m}$ so that $\left|\boldsymbol{p} \|_{<m}\right|=\{\langle\xi, k\rangle \in|\boldsymbol{p}|: k<m\}$ and $\boldsymbol{p} \|_{<m}(\xi, k)=\boldsymbol{\pi}(\xi, k)$ whenever $\langle\xi, k\rangle \in\left|\boldsymbol{p} \|_{<m}\right|$. Define $\boldsymbol{p}\left\|_{\geq m} \in \mathbf{M T}\right\|_{\geq m}, \boldsymbol{p}\left\|_{m} \in \mathbf{M T}\right\|_{m}$ similarly.

The same definitions are maintained with multiforcings:

$$
\begin{aligned}
& \text { sMF } \|_{<m}=\text { all multiforcings } \boldsymbol{\pi} \in \mathbf{s M F} \text { such that }|\boldsymbol{\pi}| \subseteq \omega_{1} \times m \text {, } \\
& \mathbf{S M F} \pi_{\geq m}=\text { all multiforcings } \boldsymbol{\pi} \in \mathbf{s M F} \text { with }|\boldsymbol{\pi}| \subseteq \omega_{1} \times(\omega \backslash m), \\
& \text { sMF } \pi_{m}=\text { all multiforcings } \boldsymbol{\pi} \in \mathbf{s M F} \text { such that }|\boldsymbol{\pi}| \subseteq \omega_{1} \times\{m\} \text {, }
\end{aligned}
$$

and $\mathbf{M F} \pi_{<m}, \mathbf{M F} \pi_{\geq m}, \mathbf{M F} \pi_{m}$ are defined similarly.

Accordingly if $\boldsymbol{\pi} \in \mathbf{M F}$ (in particular if $\boldsymbol{\pi} \in \mathbf{s M F}$ ) and $m<\omega$ then define the layer restriction $\boldsymbol{\pi}\left\|_{<m} \in \mathbf{M F}\right\|_{<m}$ (resp., $\in \mathbf{s M F} \|_{<m}$ ), so that $\left|\boldsymbol{\pi} \|_{<m}\right|=$ $\{\langle\xi, k\rangle \in|\boldsymbol{\pi}|: k<m\}$ and $\boldsymbol{\pi} \|_{<m}(\xi, k)=\boldsymbol{\pi}(\xi, k)$ whenever $\langle\xi, k\rangle \in\left|\boldsymbol{\pi} \|_{<m}\right|$. Define $\boldsymbol{\pi}\left\|_{\geq m} \in \mathbf{M F}\right\|_{\geq m}, \boldsymbol{\pi}\left\|_{m} \in \mathbf{M F}\right\|_{m}$ similarly.

A similar notation applies to multisequences (Definition 20.1). If $m<\omega$ then we let $\overrightarrow{\mathbf{s M F}}\left\|_{<m}, \overrightarrow{\mathbf{s M F}}\right\|_{\geq m}, \overrightarrow{\mathbf{s M F}} \|_{m}$ be the set of all multisequences in $\overrightarrow{\mathbf{s M F}}$ whose all terms belong to resp. $\mathbf{s M F} \pi_{<m}, \mathbf{s M F} \|_{\geq m}, \mathbf{s M F} \pi_{m}$. Define similarly $\overrightarrow{\mathbf{s M F}}_{\omega_{1}}\left\|_{<m}, \overrightarrow{\mathbf{s M F}}_{\omega_{1}}\right\|_{\geq m}, \overrightarrow{\mathbf{s M F}}_{\omega_{1}} \|_{m}$ (multisequences of length $\omega_{1}$ ).

And further, if $\overrightarrow{\boldsymbol{\pi}}=\left\langle\boldsymbol{\pi}_{\alpha}\right\rangle_{\alpha<\kappa} \in \overrightarrow{\mathbf{s M F}}$ and $m<\omega$ then define $\overrightarrow{\boldsymbol{\pi}} \|_{<m}=$ $\left\langle\boldsymbol{\pi}_{\alpha} \|_{<m}\right\rangle_{\alpha<\kappa} \in \overrightarrow{\mathbf{s M F}} \|_{<m}$, and define $\overrightarrow{\boldsymbol{\pi}}\left\|_{\geq m} \in \overrightarrow{\mathbf{s M F}}\right\|_{\geq m}, \overrightarrow{\boldsymbol{\pi}}\left\|_{m} \in \overrightarrow{\mathbf{s M F}}\right\|_{m}$ similarly. The same for $\overrightarrow{\boldsymbol{\pi}}=\left\langle\boldsymbol{\pi}_{\alpha}\right\rangle_{\alpha<\omega_{1}} \in \overrightarrow{\mathbf{s M F}}_{\omega_{1}}$

Definition 21.1. Assume that $m<\omega$. A multisequence $\overrightarrow{\boldsymbol{\pi}} \in \overrightarrow{\mathbf{s M F}} m$-decides a set $W$ if either $\overrightarrow{\boldsymbol{\pi}} \|_{\geq m}$ belongs to $W$ (positive decision) or there is no multisequence $\overrightarrow{\boldsymbol{\varphi}} \in W \cap \overrightarrow{\mathbf{s M F}} \|_{\geq m}$ extending $\overrightarrow{\boldsymbol{\pi}} \|_{\geq m}$ (negative decision).

Lemma 21.2. If $\overrightarrow{\boldsymbol{\pi}} \in \overrightarrow{\mathbf{S M F}}, \mathfrak{M}$ is countable, $W$ is any set, and $m<\omega$, then there is a multisequence $\overrightarrow{\boldsymbol{\varphi}} \in \overrightarrow{\mathbf{s M F}}$ such that $\overrightarrow{\boldsymbol{\pi}} \subset_{\mathfrak{M}} \overrightarrow{\boldsymbol{\varphi}}$ and $\overrightarrow{\boldsymbol{\varphi}}$ m-decides $W$.

Proof. By Corollary 20.2, there is a multisequence $\overrightarrow{\boldsymbol{\rho}} \in \overrightarrow{\mathbf{s M F}}$ such that $\overrightarrow{\boldsymbol{\pi}} \subset_{\mathfrak{M}}$ $\overrightarrow{\boldsymbol{\rho}}$. Then either $\overrightarrow{\boldsymbol{\rho}}$ outright $m$-decides $W$ negatively, or there is a sequence $\overrightarrow{\boldsymbol{\sigma}} \in W \cap \overrightarrow{\mathbf{s M F}} \|_{\geq m}$ satisfying $\overrightarrow{\boldsymbol{\rho}} \|_{\geq m} \subseteq \overrightarrow{\boldsymbol{\sigma}}$. 
On the other hand, using Corollary 20.2(iii), we get a multisequence $\overrightarrow{\boldsymbol{\sigma}}^{\prime} \in$ $\overrightarrow{\mathbf{s M F}} \|_{<m}$ of the same length as $\overrightarrow{\boldsymbol{\sigma}}$, such that $\overrightarrow{\boldsymbol{\rho}} \|_{<m} \subseteq \overrightarrow{\boldsymbol{\sigma}}^{\prime}$. Therefore there exists a multisequence $\overrightarrow{\boldsymbol{\varphi}} \in \overrightarrow{\mathbf{s M F}}$ of that same length, satisfying $\overrightarrow{\boldsymbol{\varphi}} \|_{>m}=\overrightarrow{\boldsymbol{\sigma}}$ and $\overrightarrow{\boldsymbol{\rho}} \|_{<m}=\vec{\sigma}^{\prime}$ - then obviously $\overrightarrow{\boldsymbol{\rho}} \subseteq \overrightarrow{\boldsymbol{\varphi}}$ and by definition $\overrightarrow{\boldsymbol{\varphi}}$ decides $W$ positively. Finally we have $\overrightarrow{\boldsymbol{\pi}} \subset_{\mathfrak{M}} \overrightarrow{\boldsymbol{\rho}} \subseteq \overrightarrow{\boldsymbol{\phi}}$, and hence $\overrightarrow{\boldsymbol{\pi}} \subset_{\mathfrak{M}} \overrightarrow{\boldsymbol{\phi}}$ by Corollary 20.2(iv).

\section{Auxiliary diamond sequences}

Recall that $\mathrm{HC}$ is the set of all hereditarily countable sets (those with finite or countable transitive closures).

The next theorem employs the technique of diamond sequences in $\mathbf{L}$.

Theorem 22.1 (in L). There exist $\Delta_{1}^{\mathrm{HC}}$ sequences $\langle\overrightarrow{\boldsymbol{\pi}}\lceil\mu\rceil\rangle_{\mu<\omega_{1}},\langle D\lceil\mu\rceil\rangle_{\mu<\omega_{1}}$, $\langle z\lceil\mu\rceil\rangle_{\mu<\omega_{1}}$, such that, for every $\mu, D\lceil\mu\rceil$ and $z\lceil\mu\rceil$ are sets in $\mathrm{HC}, \overrightarrow{\boldsymbol{\pi}}\lceil\mu\rceil \in$ $\overrightarrow{\mathbf{S M F}}, \operatorname{dom}(\overrightarrow{\boldsymbol{\pi}}\lceil\mu\rceil)=\mu$, and in addition if $\vec{\mathbb{}}=\left\langle\square_{\nu}\right\rangle_{\nu<\omega_{1}} \in \overrightarrow{\mathbf{s M F}}_{\omega_{1}}, z \in \mathrm{HC}$, and $D \subseteq \mathbf{M T}(\overrightarrow{\mathbb{T}})$, then the set $M$ of all ordinals $\mu<\omega_{1}$ such that

(a) $z\lceil\mu\rceil=z$;

(b) $\overrightarrow{\boldsymbol{\pi}}\lceil\mu\rceil$ is equal to the restricted sub-multisequence $\vec{\mathbb{}}\left\lceil\mu=\left\langle\square_{\nu}\right\rangle_{\nu<\mu}\right.$;

(c) $D\lceil\mu\rceil=D \cap \mathbf{M T}(\overrightarrow{\mathbb{\top}} \uparrow \mu)$;

is stationary in $\omega_{1}$.

Proof. Arguing in $\mathbf{L}$, the constructible universe, we let $\leqslant_{\mathbf{L}}$ be the canonical wellordering of $\mathbf{L}$. It is known that $\leqslant_{\mathbf{L}}$ orders $\mathrm{HC}$ similarly to $\omega_{1}$, and that $\leqslant_{\mathbf{L}}$ is $\Delta_{1}^{\mathrm{HC}}$ and has the goodness property: the set of all $\leqslant_{\mathbf{L}}$-initial segments $I_{x}\left(\leqslant_{\mathbf{L}}\right)=\left\{y: y \leqslant_{\mathbf{L}} x\right\}, x \in \mathrm{HC}$, is still $\Delta_{1}^{\mathrm{HC}}$.

We begin with a $\Delta_{1}^{\mathrm{HC}}$ sequence of sets $S_{\alpha} \subseteq \alpha, \alpha<\omega_{1}$, such that

(A) if $X \subseteq \mathrm{HC}$ then the set $\left\{\alpha<\omega_{1}: S_{\alpha}=X \cap \alpha\right\}$ is stationary in $\omega_{1}$.

This is a well-known instance of the diamond principle true in $\mathbf{L}$. The additional definability property can be achieved by taking the $\leqslant_{\mathbf{L}}$-least possible $S_{\alpha}$ at each step $\alpha$. We get the following two results as easy corollaries.

First, let $A_{\mu}=\left\{c_{\alpha}: \alpha \in S_{\mu}\right\}$, where $c_{\alpha}$ is the $\alpha$-th element of $\mathrm{HC}$ in the sense of the ordering $\leqslant_{\mathbf{L}}$. Then $\left\langle A_{\mu}\right\rangle_{\mu<\omega_{1}}$ is still a $\Delta_{1}^{\mathrm{HC}}$ sequence, and

(B) if $X_{\alpha} \in \mathrm{HC}$ for all $\alpha<\omega_{1}$ then the set $\left\{\mu: A_{\mu}=\left\{X_{\alpha}: \alpha<\mu\right\}\right\}$ is stationary in $\omega_{1}$.

Second, for any $\alpha$, if $A_{\alpha}=\left\langle a_{\gamma}\right\rangle_{\gamma<\alpha}$, where each $a_{\gamma}$ itself is equal to an $\omega$ sequence $\left\langle a_{\gamma}^{n}\right\rangle_{n<\omega}$, then let $B_{\alpha}^{n}=\left\langle a_{\gamma}^{n}\right\rangle_{\gamma<\alpha}$ for all $n$. Otherwise let $B_{\alpha}^{n}=\varnothing, \forall n$. Then $\left\langle B_{\alpha}\right\rangle_{\alpha<\omega_{1}}^{n<\omega}$ is still a $\Delta_{1}^{\mathrm{HC}}$ system of sets in $\mathrm{HC}$, such that 
(C) if $X_{\alpha}^{n} \in \mathrm{HC}$ for all $\alpha<\omega_{1}, n<\omega$, then, for every $\mu<\omega_{1}$, the set $\left\{\mu: \forall n\left(B_{\mu}^{n}=\left\{X_{\alpha}^{n}: \alpha<\mu\right\}\right\}\right.$ is stationary in $\omega_{1}$.

Now things become more routinely complex.

Let $\mu<\omega_{1}$. We define $z\lceil\mu\rceil=\bigcup B_{\mu}^{0}$. If $B_{\mu}^{1} \in \overrightarrow{\mathbf{s M F}}$ and $\operatorname{dom}\left(B_{\mu}^{1}\right)=\mu$ then let $\overrightarrow{\boldsymbol{\pi}}\lceil\mu\rceil=B_{\mu}^{1}$; otherwise let $\overrightarrow{\boldsymbol{\pi}}\lceil\mu\rceil$ be equal to the $\leqslant_{\mathbf{L}}$-least multisequence in $\overrightarrow{\mathbf{s M F}}$ of length $\mu$. (Those exist by Corollary 20.2(ii).) Finally we let $D\lceil\mu\rceil=\bigcup B_{\mu+1}^{2}$.

Let's show that the sequences of sets $\overrightarrow{\boldsymbol{\pi}}\lceil\mu\rceil, D\lceil\mu\rceil, z\lceil\mu\rceil$ prove the theorem. Suppose that $\overrightarrow{\mathbb{T}}=\left\langle\square_{\nu}\right\rangle_{\nu<\omega_{1}} \in \overrightarrow{\mathbf{s M F}}_{\omega_{1}}, z \in \mathrm{HC}$, and $D \subseteq \mathbf{M T}(\overrightarrow{\mathbb{\square}})$. Let $X_{\alpha}^{0}=z$, $X_{\alpha}^{1}=\left\langle\alpha, \rrbracket_{\alpha}\right\rangle, X_{\alpha}^{2}=D \cap \mathbf{M T}(\overrightarrow{\mathbb{\square}} \mid \alpha)$ for all $\alpha$. The set

$$
M=\left\{\mu<\omega_{1}: B_{\mu}^{n}=\left\{X_{\alpha}^{n}: \alpha<\mu\right\} \text { for } n=0,1,2\right\}
$$

is stationary by (C). Assume that $\mu \in M$. Then $B_{\mu}^{0}=\left\{X_{\alpha}^{0}: \alpha<\mu\right\}=\{z\}$, therefore $z\lceil\mu\rceil=z$. Further $B_{\mu}^{1}=\left\{X_{\alpha}^{1}: \alpha<\mu\right\}=\left\{\left\langle\alpha, \rrbracket_{\alpha}\right\rangle: \alpha<\mu\right\}=\vec{\Pi}\lceil\mu \in$ $\overrightarrow{\text { sMF }}$, therefore $\overrightarrow{\boldsymbol{\pi}}\lceil\mu\rceil=\overrightarrow{\mathbb{\Pi}}\left\lceil\mu\right.$. Finally we have $D\lceil\mu\rceil=\bigcup B_{\mu+1}^{2}=\bigcup_{\alpha \leq \mu} X_{\alpha}^{2}=$ $D \cap \mathbf{M T}(\overrightarrow{\mathbb{\Pi}} \uparrow \mu)$, as required.

\section{Key sequence theorem}

Now we prove a theorem which introduces the key multisequence $\vec{\mathbb{}}$.

Theorem 23.1 ( $\mathbf{V}=\mathbf{L})$. There exists a multisequence $\overrightarrow{\mathbb{\Pi}}=\left\langle\square_{\alpha}\right\rangle_{\alpha<\omega_{1}} \in \overrightarrow{\mathbf{s M F}}_{\omega_{1}}$ satisfying the following requirements:

(i) if $m<\omega$ then the multisequence $\vec{\mathbb{}} \|_{m}$ belongs to the class $\Delta_{m+2}^{\mathrm{HC}}$;

(ii) if $m^{\prime}<\omega$ and $W \subseteq \overrightarrow{\mathbf{s M F}}$ is a $\boldsymbol{\Sigma}_{m^{\prime}+1}^{\mathrm{HC}}$ set then there is an ordinal $\gamma<\omega_{1}$ such that the multisequence $\overrightarrow{\mathbb{\top}} \uparrow \gamma m^{\prime}$-decides $W$;

(iii) if a set $D \subseteq \mathbf{M T}(\overrightarrow{\mathbb{\pi}})$ is dense in $\mathbf{M T}(\overrightarrow{\mathbb{\pi}})$, then the set $Z$ of all ordinals $\gamma<\omega_{1}$ such that $\overrightarrow{\mathbb{\nabla}}\left\lceil\gamma \subset_{\{D \cap \mathbf{M T}(\overrightarrow{\mathbb{\pi}}\lceil\gamma)\}} \overrightarrow{\mathbb{\Pi}}\right.$, is stationary in $\omega_{1}$.

Proof. If $m<\omega$ then let $\mathbf{u n}_{m}(p, x)$ be a canonical universal $\Sigma_{m+1}$ formula, so that the family of all $\boldsymbol{\Sigma}_{m+1}^{\mathrm{HC}}$ sets $X \subseteq \mathrm{HC}$ (those definable in HC by $\Sigma_{m+1}$ formulas with parameters in $\mathrm{HC}$ ) is equal to the family of all sets of the form $\Upsilon_{m}(p)=\left\{x \in \mathrm{HC}: \mathrm{HC} \models \mathbf{u n}_{m}(p, x)\right\}, p \in \mathrm{HC}$.

(I) Fix $\Delta_{1}^{\mathrm{HC}}$ sequences $\langle\overrightarrow{\boldsymbol{\pi}}\lceil\mu\rceil\rangle_{\mu<\omega_{1}},\langle D\lceil\mu\rceil\rangle_{\mu<\omega_{1}}$, and $\langle z\lceil\mu\rceil\rangle_{\mu<\omega_{1}}$ satisfying Theorem 22.1; the terms $D\lceil\mu\rceil, z\lceil\mu\rceil, \overrightarrow{\boldsymbol{\pi}}\lceil\mu\rceil$ of the sequences belong to $\mathrm{HC}$, and in addition $\overrightarrow{\boldsymbol{\pi}}\lceil\mu\rceil \in \overrightarrow{\mathbf{s M F}}, \operatorname{dom}(\overrightarrow{\boldsymbol{\pi}}\lceil\mu\rceil)=\mu$.

(II) Let $\mu<\omega_{1}$. If $z\lceil\mu\rceil$ is a pair of the form $z\lceil\mu\rceil=\langle m, p\rangle$ then let $m\lceil\mu\rceil=m$ and $p\lceil\mu\rceil=p$, otherwise let $m\lceil\mu\rceil=p\lceil\mu\rceil=0$. 
(III) If $m<\omega$ then let, by Lemma $21.2, \overrightarrow{\boldsymbol{\pi}}\lceil\mu, m\rceil \in \overrightarrow{\mathbf{s M F}}$ be the $\leqslant_{\mathbf{L}}$-least multisequence in $\overrightarrow{\mathbf{s M F}}$ which satisfies $\overrightarrow{\boldsymbol{\pi}}\lceil\mu\rceil \subset_{\{D\lceil\mu\rceil\}} \overrightarrow{\boldsymbol{\pi}}\lceil\mu, m\rceil$ and $m$-decides the set $\Upsilon_{m}(p\lceil\mu\rceil)$. Let $\lceil\mu, m\rceil^{+}=\operatorname{dom}(\overrightarrow{\boldsymbol{\pi}}\lceil\mu, m\rceil)$; then $\mu<\lceil\mu, m\rceil^{+}<\omega_{1}$.

Proposition 23.2 (in $\mathbf{L}$ ). The sequences $\langle m\lceil\mu\rceil\rangle_{\mu<\omega_{1}}$ and $\langle p\lceil\mu\rceil\rangle_{\mu<\omega_{1}}$ belong to the definability class $\Delta_{1}^{\mathrm{HC}}$. If $m<\omega$ then the sequences $\langle\overrightarrow{\boldsymbol{\pi}}\lceil\mu, m\rceil\rangle_{\mu<\omega_{1}}$ and $\left\langle\lceil\mu, m\rceil^{+}\right\rangle_{\mu<\omega_{1}}$ belong to the class $\Delta_{m+2}^{\mathrm{HC}}$.

Proof. Routine. Note that $\overrightarrow{\boldsymbol{\pi}}\lceil\mu, m\rceil$ and $\lceil\mu, m\rceil^{+}$depend on $m$ through the formulas $\mathbf{u n}_{m}(\cdot, \cdot)$, whose complexity strictly increases with $m \rightarrow \infty$.

Now define a multisequence $\overrightarrow{\mathbb{T}}=\left\langle\square_{\alpha}\right\rangle_{\alpha<\omega_{1}} \in \overrightarrow{\mathbf{s M F}}_{\omega_{1}}$ and a family of strictly increasing, continuous maps $\mu_{m}: \omega_{1} \rightarrow \omega_{1}, m<\omega$, as follows:

$1^{\circ}$. Let $\mu_{m}(0)=0$ and $\mu_{m}(\lambda)=\sup _{\gamma<\lambda} \mu_{m}(\gamma)$ for all $m$ and all limit $\lambda<\omega_{1}$.

$2^{\circ}$. Suppose that $m<\omega, \gamma<\omega_{1}, \mu=\mu_{m}(\gamma)$, and the twofold-restricted sequence $\left(\overrightarrow{\mathbb{\square}}\lceil\mu) \|_{m}=\left(\overrightarrow{\mathbb{\nabla}} \|_{m}\right) \uparrow \mu\right.$ is already defined. If the following holds:

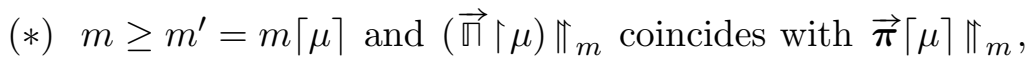

then let $\mu_{m}(\gamma+1)=\left\lceil\mu, m^{\prime}\right\rceil^{+}$and $\left(\overrightarrow{\mathbb{\Pi}}\left\lceil\left\lceil\mu, m^{\prime}\right\rceil^{+}\right) \|_{m}=\overrightarrow{\boldsymbol{\pi}}\left\lceil\mu, m^{\prime}\right\rceil \Uparrow_{m}\right.$.

$3^{\circ}$. In the assumptions of $2^{\circ}$, if $2^{\circ}(*)$ fails, then let $\overrightarrow{\boldsymbol{\rho}}$ is the $\leqslant_{\mathbf{L}}$-least multisequence in $\overrightarrow{\mathbf{s M F}}$ with $\left(\overrightarrow{\mathbb{\pi}}\lceil\mu) \|_{m} \subset \overrightarrow{\boldsymbol{\rho}}\right.$ (we refer to Corollary 20.2), and define $\mu_{m}(\gamma+1)=\operatorname{dom}(\overrightarrow{\boldsymbol{\rho}})$ and $\left(\overrightarrow{\mathbb{T}}\left\lceil\mu_{m}(\gamma+1)\right) \Uparrow_{m}=\overrightarrow{\boldsymbol{\rho}} \|_{m}\right.$.

To conclude, given $\gamma<\omega_{1}$ and $m$, if an ordinal $\mu=\mu_{m}(\gamma)$, and a multisequence $\left(\overrightarrow{\mathbb{T}}\lceil\mu) \|_{m}=\left(\overrightarrow{\mathbb{\nabla}} \|_{m}\right)\left\lceil\mu\right.\right.$ are defined, then items $2^{\circ}, 3^{\circ}$ define a bigger ordinal $\mu_{m}(\gamma+1)>\mu=\mu_{m}(\gamma)$ and a longer multisequence $\left(\overrightarrow{\mathbb{G}} \uparrow \mu_{m}(\gamma+1)\right) \|_{m}$ satisfying $\left(\overrightarrow{\mathbb{\pi}}\lceil\mu) \|_{m} \subset\left(\overrightarrow{\mathbb{\pi}}\left\lceil\mu_{m}(\gamma+1)\right) \|_{m}\right.\right.$. Thus overall items $1^{\circ}, 2^{\circ}, 3^{\circ}$ of the definition contain straightforward instructions as how to uniquely define the layers $\overrightarrow{\mathbb{\pi}} \|_{m}$ and maps $\mu_{m}$ for different $m<\omega$, independently from each other.

From now on, fix a multisequence $\vec{\mathbb{}}=\left\langle\square_{\alpha}\right\rangle_{\alpha<\omega_{1}} \in \overrightarrow{\mathbf{s M F}}_{\omega_{1}}$ of multiforcings $\mathbb{\square}_{\alpha} \in \mathbf{s M F}$ and increasing continuous maps $\mu_{m}: \omega_{1} \rightarrow \omega_{1}$ defined by $1^{\circ}, 2^{\circ}, 3^{\circ}$. As the maps $\mu_{m}$ are continuous, the following holds:

Proposition 23.3 (in $\mathbf{L}) . \mathbb{C}=\left\{\gamma<\omega_{1}: \forall m\left(\gamma=\mu_{m}(\gamma)\right)\right\}$ is a club in $\omega_{1}$.

To show that $\overrightarrow{\mathbb{T}}$ proves Theorem 23.1, we check items (i), (ii), (iii).

(i) Let $m<\omega$. Then the multisequence $\overrightarrow{\mathbb{\pi}} \|_{m}$ and the map $\mu_{m}$ belong to the class $\Delta_{m+2}^{\mathrm{HC}}$ by Proposition 23.2; a routine proof is omitted.

(ii) Suppose that $m^{\prime}<\omega$ and $W \subseteq \overrightarrow{\mathbf{s M F}}$ is a $\boldsymbol{\Sigma}_{m^{\prime}+2}^{\mathrm{HC}}$ set. Pick $p \in \mathrm{HC}$ such that $W=\Upsilon_{m^{\prime}}(p)$. Let $z=\left\langle m^{\prime}, p\right\rangle$. As $\mathbb{C}$ is a club, it follows from the choice of 
terms $\overrightarrow{\boldsymbol{\pi}}\lceil\mu\rceil, D\lceil\mu\rceil$, and $z\lceil\mu\rceil$, by (I) and Theorem 22.1, that there is an ordinal $\gamma \in \mathbb{C}$ such that $\overrightarrow{\boldsymbol{\pi}}\lceil\gamma\rceil=\overrightarrow{\mathbb{\Pi}}\left\lceil\gamma\right.$ and $z\lceil\gamma\rceil=z$ - hence, $m\lceil\gamma\rceil=m^{\prime}$ and $p\lceil\gamma\rceil=p$.

Let $\mu=\gamma$; then also $\mu=\mu_{m}(\gamma), \forall m-$ since $\gamma \in \mathbb{C}$, and $\overrightarrow{\mathbb{\Pi}}\lceil\mu=\overrightarrow{\boldsymbol{\pi}}\lceil\mu\rceil$.

Then it follows from the choice of $\vec{\mathbb{}}$ that item $2^{\circ}$ of the construction applies for the ordinal $\gamma$ chosen and all $m \geq m^{\prime}$. It follows that the multisequence $\overrightarrow{\boldsymbol{\rho}}=\overrightarrow{\boldsymbol{\pi}}\left\lceil\mu, m^{\prime}\right\rceil$ and the ordinal $\nu=\mu_{m}(\gamma+1)=\left\lceil\mu, m^{\prime}\right\rceil^{+}$satisfy $\nu=\operatorname{dom}(\overrightarrow{\boldsymbol{\rho}})$ and $\left(\overrightarrow{\mathbb{\nabla}}\lceil\nu)\left\|_{m}=\overrightarrow{\boldsymbol{\rho}}\right\|_{m}\right.$ for all $m \geq m^{\prime}$. In other words, $\left(\overrightarrow{\mathbb{\nabla}}\lceil\nu)\left\|_{\geq m^{\prime}}=\overrightarrow{\boldsymbol{\rho}}\right\|_{\geq m^{\prime}}\right.$.

However by definition $\overrightarrow{\boldsymbol{\rho}} m^{\prime}$-decides the set $W=\Upsilon_{m^{\prime}}(p)$, and the definition of this property depends only on $\overrightarrow{\boldsymbol{\rho}} \|_{\geq m^{\prime}}$.

(iii) Assume that a set $D \subseteq \mathbf{M T}(\overrightarrow{\mathbb{\pi}})$ is dense in $\mathbf{M T}(\overrightarrow{\mathbb{\pi}})$, and $C \subseteq \mathbb{C}$ is a club in $\omega_{1}$. Following the proof of (ii), we find an ordinal $\gamma \in C$ such that $\overrightarrow{\boldsymbol{\pi}}\lceil\gamma\rceil=\overrightarrow{\mathbb{\Pi}}\left\lceil\gamma, m\lceil\gamma\rceil=0\right.$, and $D\lceil\gamma\rceil=D \cap \mathbf{M T}\left(\overrightarrow{\mathbb{\pi}}\lceil\gamma)\right.$, Note that $\gamma=\mu_{m}(\gamma)$, $\forall m$. We have $\overrightarrow{\boldsymbol{\pi}}\lceil\gamma\rceil \subset_{\{D\lceil\gamma\rceil\}} \overrightarrow{\boldsymbol{\pi}}\lceil\gamma, 0\rceil$ by (III) (with $\mu=\gamma$ ), that is,

$$
\overrightarrow{\boldsymbol{\pi}}\lceil\gamma\rceil \subset_{\{D \cap \mathbf{M T}(\overrightarrow{\mathbb{\pi}}\lceil\gamma)\}} \overrightarrow{\boldsymbol{\pi}}\lceil\gamma, 0\rceil .
$$

Yet it follows from the choice of $\gamma$ that condition $2^{\circ}(*)$ holds (for $\mu=\gamma$ ) for all $m \geq 0$. Then, by definition $2^{\circ}$, the ordinal $\mu^{+}=\lceil\gamma, m\rceil^{+}$satisfies $\mu^{+}=$ $\mu_{m}(\gamma+1)$ and $\left(\overrightarrow{\mathbb{\pi}}\left\lceil\mu^{+}\right)\left\|_{m}=(\overrightarrow{\boldsymbol{\pi}}\lceil\gamma, 0\rceil)\right\|_{m}\right.$ for all $m$, that is, just $\overrightarrow{\mathbb{\pi}}\left\lceil\mu^{+}=\right.$ $\overrightarrow{\boldsymbol{\pi}}\lceil\gamma, 0\rceil$. We conclude that $\overrightarrow{\mathbb{\pi}}\left\lceil\gamma \subset_{\{D \cap \mathbf{M T}(\overrightarrow{\mathbb{\pi}} \mid \gamma)\}} \overrightarrow{\mathbb{\Pi}\lceil}\left\lceil\mu^{+}\right.\right.$by $(\dagger)$, therefore we have $\overrightarrow{\mathbb{\pi}}\left\lceil\gamma \subset_{\{D \cap \mathbf{M T}(\overrightarrow{\mathbb{\pi}} \mid \gamma)\}} \overrightarrow{\mathbb{\pi}}\right.$, as required.

(Theorem 23.1)

Definition 23.4 (in $\mathbf{L}$ ). From now on we fix a multisequence $\vec{\mathbb{}}=\left\langle\square_{\alpha}\right\rangle_{\alpha<\omega_{1}} \in$ $\overrightarrow{\mathbf{s M F}}_{\omega_{1}}$ satisfying requirements of Theorem 23.1, that is,

(i) if $m<\omega$ then the multisequence $\overrightarrow{\mathbb{\top}} \|_{m}$ belongs to the class $\Delta_{m+2}^{\mathrm{HC}}$;

(ii) if $m^{\prime}<\omega$ and $W \subseteq \overrightarrow{\mathbf{s M F}}$ is a $\boldsymbol{\Sigma}_{m^{\prime}+1}^{\mathrm{HC}}$ set then there is an ordinal $\gamma<\omega_{1}$ such that the multisequence $\vec{\mathbb{}} \uparrow \gamma m^{\prime}$-decides $W$;

(iii) if a set $D \subseteq \mathbf{M T}(\overrightarrow{\mathbb{\pi}})$ is dense in $\mathbf{M T}(\overrightarrow{\mathbb{\pi}})$, then the set $Z$ of all ordinals $\gamma<\omega_{1}$ such that $\overrightarrow{\mathbb{\pi}}\left\lceil\gamma \subset_{\{D \cap \mathbf{M T}(\overrightarrow{\mathbb{\pi}}\lceil\gamma)\}} \overrightarrow{\mathbb{\pi}}\right.$, is stationary in $\omega_{1}$.

We call $\overrightarrow{\mathbb{T}}$ the key multisequence.

A set $U \subseteq \mathbf{S M F} \pi_{\geq m}$ is dense in $\mathbf{s M F} \pi_{\geq m}$ if for each $\overrightarrow{\boldsymbol{\pi}} \in \mathbf{s M F} \|_{\geq m}$ there is a multisequence $\overrightarrow{\boldsymbol{\varphi}} \in U$ satisfying $\overrightarrow{\boldsymbol{\pi}} \subseteq \overrightarrow{\boldsymbol{\varphi}}$.

Lemma 23.5. If $m<\omega$ and $W \subseteq \mathbf{s M F} \|_{\geq m}$ is a $\boldsymbol{\Sigma}_{m+1}^{\mathrm{HC}}$ set dense in $\mathbf{s M F} \|_{\geq m}$ then there is an ordinal $\gamma<\omega_{1}$ such that $(\overrightarrow{\mathbb{\pi}} \uparrow \gamma) \|_{\geq m} \in W$. In particular, if $W \subseteq \overrightarrow{\mathbf{S M F}}$ is a $\mathbf{\Sigma}_{1}^{\mathrm{HC}}$ set dense in $\overrightarrow{\mathbf{s M F}}$ then there is $\gamma<\omega_{1}$ such that $\vec{\square}\lceil\gamma \in W$.

Proof. Apply 23.4(ii). The negative decision is impossible by the density. 


\section{Key product forcing}

We continue to argue in $\mathbf{L}$, and we'll make use of the key multisequence $\vec{\mathbb{}}=$ $\left\langle\square_{\alpha}\right\rangle_{\alpha<\omega_{1}}$ introduced by Definition 23.4.

Definition 24.1 (in $\mathbf{L}$ ). Define the multiforcings

$$
\begin{aligned}
& \square=\bigcup^{\mathrm{cw}} \vec{\Pi} \quad=\bigcup_{\alpha<\omega_{1}}^{\mathrm{cw}} \square_{\alpha} \in \mathbf{M F}, \\
& \square_{<\gamma}=\bigcup^{\mathrm{cw}}(\overrightarrow{\mathbb{\Pi}} \uparrow \gamma) \quad=\bigcup_{\alpha<\gamma}^{\mathrm{cw}} \rrbracket_{\alpha} \quad \in \text { sMF, for each } \gamma<\omega_{1} \\
& \square_{\geq \gamma}=\bigcup^{\mathrm{cw}}\left(\overrightarrow{\mathbb{\Pi} \uparrow}\left(\omega_{1} \backslash \gamma\right)\right)=\bigcup_{\gamma \leq \alpha<\omega_{1}}^{\mathrm{cw}} \rrbracket_{\alpha} \in \mathbf{M F} \text {, for each } \gamma<\omega_{1} \text {. }
\end{aligned}
$$

We further define $\mathbb{P}=\mathbf{M T}(\mathbb{\square})=\mathbf{M T}(\overrightarrow{\mathbb{\Pi}})$, and, for all $\gamma<\omega_{1}$,

$$
\mathbb{P}_{<\gamma}=\mathbf{M T}\left(\square_{<\gamma}\right)=\mathbf{M T}(\overrightarrow{\mathbb{\pi} \uparrow \gamma}), \quad \mathbb{P}_{\geq \gamma}=\mathbf{M T}\left(\square_{\geq \gamma}\right)=\mathbf{M T}\left(\overrightarrow{\mathbb{\pi}} \uparrow\left(\omega_{1} \backslash \gamma\right)\right) .
$$

The multiforcing $\mathbb{P}$ will be our principal forcing notion, the key forcing.

Lemma 24.2 (in $\mathbf{L}$ ). $\square$ is a regular multiforcing. In addition, $|\square|=\omega_{1} \times \omega$, thus if $\xi<\omega_{1}$ and $k<\omega$ then there is an ordinal $\alpha<\omega_{1}$ such that $\langle\xi, k\rangle \in\left|\square_{\alpha}\right|$. Therefore $\mathbb{P}=\prod_{\xi<\omega_{1}, k<\omega} \square(\xi, k)$ (with finite support).

Proof. To prove the additional claim, note that the set $W$ of all multisequences $\overrightarrow{\boldsymbol{\pi}} \in \overrightarrow{\mathbf{s M F}}$ satisfying $\langle\xi, k\rangle \in\left|\bigcup^{\mathrm{cw}} \overrightarrow{\boldsymbol{\pi}}\right|$ is $\boldsymbol{\Sigma}_{1}^{\mathrm{HC}}$ (with $\xi$ as a parameter of definition). In addition $W$ is dense in $\overrightarrow{\mathbf{s M F}}$. (First extend $\overrightarrow{\boldsymbol{\pi}}$ by Corollary 20.2 so that is has a non-limit length and the last term, then make use of Corollary 11.4.) Therefore by Lemma 23.5 there is an ordinal $\gamma<\omega_{1}$ such that $\vec{\mathbb{}}\lceil\gamma \in W$, as required.

If $\xi<\omega_{1}$ and $k<\omega$ then, following the lemma, let $\alpha(\xi, k)<\omega_{1}$ be the least ordinal $\alpha$ satisfying $\langle\xi, k\rangle \in\left|\mathbb{\square}_{\alpha}\right|$. Thus a forcing $\square_{\alpha}(\xi, k) \in \mathbf{P T F}$ is defined whenever $\alpha$ satisfies $\alpha(\xi, k) \leq \alpha<\omega_{1}$, and $\left\langle\square_{\alpha}(\xi, k)\right\rangle_{\alpha(\xi, k) \leq \alpha<\omega_{1}}$ is a $\sqsubset$ increasing sequence of countable special forcings in PTF.

Note that $\square(\xi, k)=\bigcup_{\alpha(\xi, k) \leq \alpha<\omega_{1}} \rrbracket_{\alpha}(\xi, k)$ by construction.

Corollary 24.3 (in $\mathbf{L}$ ). If $k<\omega$ then the sequence of ordinals $\langle\alpha(\xi, k)\rangle_{\xi<\omega_{1}}$ and the sequence of multiforcings $\left\langle\square_{\alpha}(\xi, k)\right\rangle_{\xi<\omega_{1}, \alpha(\xi, k) \leq \alpha<\omega_{1}}$ are $\Delta_{k+2}^{\mathrm{HC}}$.

Proof. By construction the following double equivalence holds:

$$
\begin{aligned}
\alpha<\alpha(\xi, k) & \Longleftrightarrow \exists \boldsymbol{\pi}\left(\boldsymbol{\pi}=\mathbb{\square}_{\alpha} \|_{k} \wedge\langle\xi, k\rangle \in \operatorname{dom} \boldsymbol{\pi}\right) \quad \Longleftrightarrow \\
& \Longleftrightarrow \forall \boldsymbol{\pi}\left(\boldsymbol{\pi}=\mathbb{\square}_{\alpha} \|_{k} \Longrightarrow\langle\xi, k\rangle \in \operatorname{dom} \boldsymbol{\pi}\right)
\end{aligned}
$$

However $\boldsymbol{\pi}=\mathbb{\square}_{\alpha} \rrbracket_{k}$ is a $\Delta_{k+2}^{\mathrm{HC}}$ relation by Theorem 23.1(i). It follows that so is the sequence $\langle\alpha(\xi, k)\rangle_{\xi<\omega_{1}}$. The second claim easily follows by the same Definition 23.4(i). 
Corollary 24.4 (in $\mathbf{L}$, of Lemma 9.2(v)). If $\xi<\omega_{1}, k<\omega$, and $\alpha(\xi, k) \leq \alpha<$ $\omega_{1}$ then the set $\square_{\alpha}(\xi, k)$ is pre-dense in $\square(\xi, k)$ and in $\square$.

In spite of Lemma 24.2, the sets $\left|\rrbracket_{<\gamma}\right|$ can be quite arbitrary (countable) subsets of $\omega_{1} \times \omega$. However we easily get the next corollary:

Corollary 24.5 (in $\mathbf{L}$, of Lemma 24.2). The set $\mathbb{C}^{\prime}=\left\{\gamma<\omega_{1}:\left|\square_{<\gamma}\right|=\gamma \times \omega\right\}$ is a club in $\omega_{1}$.

Lemma 24.6 (in $\mathbf{L}$ ). $\mathbb{P}$ is $C C C$.

Proof. Let $A \subseteq \mathbb{P}$ be a maximal antichain in $\mathbb{P}$. The set

$$
C=\left\{\gamma<\omega_{1}: A \cap \mathbb{P}_{<\gamma} \text { is a maximal antichain in } \mathbb{P}_{<\gamma}\right\}
$$

is a club in $\omega_{1}$. Let $D=\{\boldsymbol{p} \in \mathbb{P}: \exists \boldsymbol{q} \in A(p \leqslant q)\}$; this is an open dense set. By Definition 23.4(iii), there is an ordinal $\gamma \in C$ such that $\vec{\pi}\left\lceil\gamma \subset_{\{D \cap \mathbb{P}<\gamma\}} \overrightarrow{\mathbb{\pi}}\right.$. Recall that $\gamma \in C$, hence $A \cap \mathbb{P}_{<\gamma}$ is a maximal antichain in $\mathbb{P}_{<\gamma}$, thus $D \cap \mathbb{P}_{<\gamma}$ is open dense in $\mathbb{P}_{<\gamma}$. Therefore the set $D \cap \mathbb{P}_{<\gamma}$ is pre-dense in the forcing $\mathbf{M T}(\overrightarrow{\mathbb{\pi}})=\mathbb{P}$ by Corollary 20.2(v)(c). We claim that $A=A \cap \mathbb{P}_{<\gamma}$, so $A$ is countable.

Indeed suppose that $\boldsymbol{r} \in A \backslash \mathbb{P}_{<\gamma}$. Then $\boldsymbol{r}$ is compatible with some $\boldsymbol{q} \in$ $D \cap \mathbb{P}_{<\gamma}$; let $\boldsymbol{p} \in D \cap \mathbb{P}_{<\gamma}, \boldsymbol{p} \leqslant \boldsymbol{q}, \boldsymbol{p} \leqslant \boldsymbol{r}$. As $\boldsymbol{q} \in D$, there is some $\boldsymbol{r}^{\prime} \in A$ with $\boldsymbol{q} \leqslant \boldsymbol{r}^{\prime}$. Then $\boldsymbol{r}=\boldsymbol{r}^{\prime}$ as $A$ is an antichain; thus $\boldsymbol{q} \leqslant \boldsymbol{r} \in A \backslash \mathbb{P}_{<\gamma}$. However $\boldsymbol{q} \in \mathbb{P}_{<\gamma}$ and $A \cap \mathbb{P}_{<\gamma}$ is a maximal antichain in $\mathbb{P}_{<\gamma}$, thus $\boldsymbol{q}$, and hence $\boldsymbol{r}$, is compatible with some $\boldsymbol{r}^{\prime \prime} \in A \cap \mathbb{P}_{<\gamma}$. Which is a contradiction.

Corollary 24.7 (in $\mathbf{L}$ ). If a set $D \subseteq \mathbb{P}$ is pre-dense in $\mathbb{P}$ then there is an ordinal $\gamma<\omega_{1}$ such that $D \cap \mathbb{P}_{<\gamma}$ is already pre-dense in $\mathbb{P}$.

Proof. We can assume that in fact $D$ is dense. Let $A \subseteq D$ be a maximal antichain in $D$; then $A$ is a maximal antichain in $\mathbb{P}$ because of the density of $D$. Then $A \subseteq \mathbb{P}_{<\gamma}$ for some $\gamma<\omega_{1}$ by Lemma 24.6. But $A$ is pre-dense in $\mathbb{P}$. 


\section{Auxiliary forcing relation}

Recall that $\mathbb{P}=\mathbf{M T}(\mathbb{\square})$, the key forcing, is a product forcing notion defined (in $\mathbf{L}$ ) in Section 24. Its components $\square(\xi, k)$ have different complexity in $\mathrm{HC}$, depending on $k$ by Corollary 24.3, hence there is no way the forcing notion $\mathbb{P}$ (or $\square)$ as a whole is definable in HC. Somewhat surprisingly, the $\mathbb{P}$-forcing relation turns out to be definable in $\mathrm{HC}$ when restricted to analytic formulas of a certain level of complexity within the usual hierarchy. This will be established on the base of an auxiliary forcing relation.

\section{Auxiliary forcing: preliminaries}

We argue in $\mathbf{L}$. Consider the 2nd order arithmetic language, with variables $k, l, m, n, \ldots$ of type 0 over $\omega$ and variables $a, b, x, y, \ldots$ of type 1 over $\omega^{\omega}$, whose atomic formulas are those of the form $x(k)=n$. Let $\mathscr{L}$ be the extension of this language, which allows to substitute free variables of type 0 with natural numbers (as usual) and free variables of type 1 with small real names $\mathbf{c} \in \mathbf{L}$. By $\mathscr{L}$-formulas we understand formulas of this extended language.

We define natural classes $\mathscr{L} \Sigma_{n}^{1}, \mathscr{L} \Pi_{n}^{1}(n \geq 1)$ of $\mathscr{L}$-formulas. Let $\mathscr{L}(\Sigma+\Pi)_{1}^{1}$ be the closure of $\mathscr{L} \Sigma_{1}^{1} \cup \mathscr{L} \Pi_{1}^{1}$ under $\neg, \wedge, \vee$ and quantifiers over $\omega$. If $\varphi$ is a formula in $\mathscr{L} \Sigma_{n}^{1}$ (resp., $\mathscr{L} \Pi_{n}^{1}$ ), then let $\varphi^{-}$be the result of canonical transformation of $\neg \varphi$ to the $\mathscr{L} \Pi_{n}^{1}$ (resp., $\mathscr{L} \Sigma_{n}^{1}$ ) form.

If $\varphi$ is a $\mathscr{L}$-formula and $G \subseteq \mathbf{M T}$ is a pairwise compatible set of multitrees then let $\varphi[G]$ be the result of substitution of $\mathbf{c}[G]$ for any name $\mathbf{c}$ in $\varphi$. (Recall Definition 13.2.) Thus $\varphi[G]$ is an ordinary 2 nd order arithmetic formula, which may include natural numbers and elements of $\omega^{\omega}$ as parameters.

We are going to define a relation $\boldsymbol{p} \operatorname{forc}_{\vec{\pi}} \varphi$ between multitrees $\boldsymbol{p}$, multisequences $\overrightarrow{\boldsymbol{\pi}}$, and $\mathscr{L}$-formulas $\varphi$, which suitably approximates the true $\mathbb{P}$-forcing relation. But it depends on a two more definitions.

Definition 25.1. If $m<\omega$ then $\overrightarrow{\mathbf{s M F}}\left[\vec{\pi} \|_{<m}\right]$ consists of all multisequences $\overrightarrow{\boldsymbol{\pi}} \in \overrightarrow{\mathbf{s M F}}$ such that $\overrightarrow{\boldsymbol{\pi}}\left\|_{<m} \subset \overrightarrow{\mathbb{\pi}}\right\|_{<m}$, that is, $\overrightarrow{\boldsymbol{\pi}} \|_{<m}=\left(\overrightarrow{\mathbb{\Pi}} \|_{<m}\right)\lceil\delta$, where $\delta=$ $\operatorname{dom}(\overrightarrow{\boldsymbol{\pi}})$ - multisequences which agree with the key multisequence $\overrightarrow{\mathbb{\pi}}$ on layers below $m$. Obviously $\overrightarrow{\mathbf{s M F}}\left[\overrightarrow{\mathbb{\Pi}} \|_{<m+1}\right] \subseteq \overrightarrow{\mathbf{s M F}}\left[\vec{\Pi} \|_{<m}\right] \subseteq \overrightarrow{\mathbf{s M F}}\left[\vec{\Pi} \|_{<0}\right]=\overrightarrow{\mathbf{s M F}}$.

If $\gamma<\omega_{1}$ then the subsequence $\vec{\mathbb{}} \uparrow \gamma$ of the key multisequence $\vec{\pi}$ belongs to $\bigcap_{m} \overrightarrow{\mathbf{s M F}}\left[\vec{\Pi} \|_{<m}\right]$, of course. To prove the next lemma use 23.4(i).

Lemma 25.2. $\overrightarrow{\mathbf{s M F}}\left[\vec{\pi} \|_{<m}\right]$ is a subset of $\mathrm{HC}$ of definability class $\Delta_{m+1}^{\mathrm{HC}}$.

The other definition deals with models of a subtheory of ZFC. 
Definition 25.3. Let $\mathbf{Z F L}^{-}$be the theory containing all axioms of $\mathbf{Z F C}^{-}$(minus the Power Set axiom) plus the axiom of constructibility $\mathbf{V}=\mathbf{L}$. Any transitive model (TM) of $\mathbf{Z F L}^{-}$has the form $\mathbf{L}_{\alpha}$, where $\alpha \in$ Ord. Therefore it is true in $\mathbf{L}$ that for any set $x$ there is a least $\mathrm{TM} \mathfrak{M}=\mathfrak{M}(x)$ of $\mathbf{Z F L}^{-}$containing $x$. If $x \in \mathrm{HC}(\mathrm{HC}=$ all hereditarily countable sets) then $\mathfrak{M}(x)$ is a countable transitive model (CTM), equal to the least CTM of $\mathbf{Z F L}^{-}$containing $x$.

\section{Auxiliary forcing}

The definition of $\boldsymbol{p}$ forc $\overrightarrow{\boldsymbol{\pi}} \varphi$ goes on by induction on the complexity of $\varphi$.

$1^{\circ}$. Let $\varphi$ is a closed $\mathscr{L}(\Sigma+\Pi)_{1}^{1}$ formula, $\overrightarrow{\boldsymbol{\pi}} \in \overrightarrow{\mathbf{s M F}}, \boldsymbol{p} \in \mathbf{M T}$, but $\boldsymbol{p} \in \mathbf{M T}(\overrightarrow{\boldsymbol{\pi}})$ is not necessarily assumed. We define:

(a) $\boldsymbol{p} \operatorname{forc}_{\vec{\pi}} \varphi$ iff there is a $\mathrm{CTM} \mathfrak{M} \models \mathbf{Z F L}^{-}$, an ordinal $\vartheta<\operatorname{dom} \overrightarrow{\boldsymbol{\pi}}$, and a multitree $\boldsymbol{p}_{0} \in \mathbf{M T}\left(\overrightarrow{\boldsymbol{\pi}}\lceil\vartheta)\right.$, such that $\boldsymbol{p} \leqslant \boldsymbol{p}_{0}$ (meaning: $\boldsymbol{p}$ is stronger), the model $\mathfrak{M}$ contains $\overrightarrow{\boldsymbol{\pi}}\lceil\vartheta$ (then contains $\mathbf{M T}(\overrightarrow{\boldsymbol{\pi}}\lceil\vartheta)$ as well) and contains $\varphi$ (that is, all names in $\varphi$ ), $\overrightarrow{\boldsymbol{\pi}}\left\lceil\vartheta \subset_{\mathfrak{M}} \overrightarrow{\boldsymbol{\pi}}\right.$, and $\boldsymbol{p}_{0}$ $\operatorname{MT}(\overrightarrow{\boldsymbol{\pi}}\lceil\vartheta)$-forces $\varphi[\underline{G}]$ over $\mathfrak{M}$ (in the usual sense) ;

(b) $\boldsymbol{p}$ wforc $\overrightarrow{\boldsymbol{\pi}} \varphi$ (weak forcing) iff there is no multisequence $\overrightarrow{\boldsymbol{\pi}}^{\prime} \in \overrightarrow{\mathbf{s M F}}$ and $\boldsymbol{p}^{\prime} \in \mathbf{M T}\left(\overrightarrow{\boldsymbol{\pi}}^{\prime}\right)$ such that $\overrightarrow{\boldsymbol{\pi}} \subseteq \overrightarrow{\boldsymbol{\pi}}^{\prime}, \boldsymbol{p}^{\prime} \leqslant \boldsymbol{p}$, and $\boldsymbol{p}^{\prime}$ forc $\overrightarrow{\boldsymbol{\pi}}^{\prime} \neg \varphi$.

$2^{\circ}$. If $\varphi(x)$ is a $\mathscr{L} \Pi_{n}^{1}$ formula, $n \geq 1$, then we define $\boldsymbol{p}$ forc $\overrightarrow{\boldsymbol{\pi}} \exists x \varphi(x)$ iff there is a small real name $\mathbf{c}$ such that $\boldsymbol{p}$ forc $\vec{\pi} \varphi(\mathbf{c})$.

$3^{\circ}$. If $\varphi$ is a closed $\mathscr{L} \Pi_{n}^{1}$ formula, $n \geq 2$, then we define $\boldsymbol{p} \operatorname{forc}_{\vec{\pi}} \varphi$ iff $\overrightarrow{\boldsymbol{\pi}} \in$ $\overrightarrow{\mathbf{s M F}}\left[\overrightarrow{\mathbb{\Pi}} \|_{<n-2}\right]$, and there is no multisequence $\overrightarrow{\boldsymbol{\pi}}^{\prime} \in \overrightarrow{\mathbf{s M F}}\left[\overrightarrow{\mathbb{\pi}} \pi_{<n-2}\right]$ and multitree $\boldsymbol{p}^{\prime} \in \mathbf{M T}\left(\overrightarrow{\boldsymbol{\pi}}^{\prime}\right)$ such that $\overrightarrow{\boldsymbol{\pi}} \subseteq \overrightarrow{\boldsymbol{\pi}}^{\prime}, \boldsymbol{p}^{\prime} \leqslant \boldsymbol{p}$, and $\boldsymbol{p}^{\prime}$ forc $\overrightarrow{\boldsymbol{\pi}}^{\prime} \varphi^{-} .5$

Remark 26.1. With $\boldsymbol{p}_{0}$ and $\vartheta$ given, the premise " $\boldsymbol{p}_{0} \mathbf{M T}(\overrightarrow{\boldsymbol{\pi}}\lceil\vartheta)$-forces $\varphi[\underline{G}]$ over $\mathfrak{M}$ " of $1^{\circ}$ a does not depend on the choice of a CTM $\mathfrak{M}$ containing $\overrightarrow{\boldsymbol{\pi}}\lceil\vartheta$ and $\varphi$, since if $\varphi$ is $\mathscr{L}(\Sigma+\Pi)_{1}^{1}$ then the formula $\varphi[G]$ (in which all names are evaluated by some $\mathbf{M T}(\overrightarrow{\boldsymbol{\pi}}\lceil\vartheta)$-generic set $G$ as in 13.2) in simultaneously true or false in all transitive models by the Shoenfield absoluteness theorem.

Remark 26.2. It easily holds by induction that if $\boldsymbol{p}$ forc $_{\vec{\pi}} \varphi$ then $\overrightarrow{\boldsymbol{\pi}} \in \overrightarrow{\mathbf{s M F}}$, $\varphi$ is a closed formula in one of the classes $\mathscr{L}(\Sigma+\Pi)_{1}^{1}, \mathscr{L} \Sigma_{n}^{1}, \mathscr{L} \Pi_{n}^{1}, n \geq 2$, and if $n \geq 2$ and $\varphi \in \mathscr{L} \Pi_{n}^{1} \cup \mathscr{L} \Sigma_{n+1}^{1}$ then $\overrightarrow{\boldsymbol{\pi}} \in \overrightarrow{\mathbf{s M F}}\left[\vec{\pi} \|_{<n-2}\right]$.

\footnotetext{
${ }^{5}$ If $\overrightarrow{\boldsymbol{\pi}}$ does not belong to $\overrightarrow{\mathbf{s M F}}\left[\overrightarrow{\mathbb{\pi}} \|_{<n-2}\right]$ in $3^{\circ}$, then $\boldsymbol{p}$ forc $\overrightarrow{\boldsymbol{\pi}} \varphi$ holds for any $\mathscr{L} \Pi_{n}^{1}$ formula $\varphi$ by default as a multisequence not in $\overrightarrow{\mathbf{s M F}}\left[\overrightarrow{\mathbb{\pi}} \pi_{<n-2}\right]$ is definitely not extendable to a multisequence in $\overrightarrow{\mathbf{s M F}}\left[\overrightarrow{\mathbb{\pi}} \|_{<n-2}\right]$. This motivates the condition $\overrightarrow{\boldsymbol{\pi}} \in \overrightarrow{\mathbf{s M F}}\left[\overrightarrow{\mathbb{\pi}} \|_{<n-2}\right]$ in $3^{\circ}$.
} 
Lemma 26.3. Assume that multisequences $\overrightarrow{\boldsymbol{\pi}} \subseteq \overrightarrow{\boldsymbol{\varphi}}$ belong to $\overrightarrow{\mathbf{s M F}}, \boldsymbol{q}, \boldsymbol{p} \in \mathbf{M T}$, $\boldsymbol{q} \leqslant \boldsymbol{p}, \varphi$ is an $\mathscr{L}$-formula as in 26.2, and if $n \geq 2$ and $\varphi \in \mathscr{L} \Pi_{n}^{1} \cup \mathscr{L} \Sigma_{n+1}^{1}$ then $\overrightarrow{\boldsymbol{\pi}}, \overrightarrow{\boldsymbol{\phi}} \in \overrightarrow{\mathbf{s M F}}\left[\overrightarrow{\mathbb{\top}} \|_{<n-2}\right]$. Then $\boldsymbol{p} \operatorname{forc}_{\overrightarrow{\boldsymbol{\pi}}} \varphi$ implies $\boldsymbol{q} \operatorname{forc}_{\overrightarrow{\boldsymbol{\phi}}} \varphi$, and if $\varphi$ belongs to $\mathscr{L}(\Sigma+\Pi)_{1}^{1}$ then $\boldsymbol{p}$ wforc $_{\vec{\pi}} \varphi$ implies $\boldsymbol{q}$ wforc $\vec{\phi} \varphi$ as well.

Proof. If $\varphi$ is a $\mathscr{L}(\Sigma+\Pi)_{1}^{1}$ formula, $\boldsymbol{p} \operatorname{forc} \vec{\pi} \varphi$, and this is witnessed by $\mathfrak{M}$, $\vartheta, \boldsymbol{p}_{0}$ as in $1^{\circ}$ a, then the exactly same $\mathfrak{M}, \vartheta, \boldsymbol{p}_{0}$ witness $\boldsymbol{q}$ forc $\vec{\phi} \varphi$.

The induction step $\exists$, as in $2^{\circ}$, is elementary.

Now the induction step $\forall$, as in $3^{\circ}$. Let $\varphi$ be a closed $\mathscr{L} \Pi_{n}^{1}$-formula, $n \geq 2$, and $\boldsymbol{p} \operatorname{forc}_{\vec{\pi}} \varphi$. Assume to the contrary that $\boldsymbol{q} \operatorname{forc}_{\vec{\phi}} \varphi$ fails. Then by $3^{\circ}$ there exist: a multisequence $\overrightarrow{\boldsymbol{\varphi}}^{\prime} \in \overrightarrow{\mathbf{s M F}}\left[\overrightarrow{\mathbb{\pi}} \|_{<n-2}\right]$ and multitree $\boldsymbol{q}^{\prime} \in \mathbf{M T}\left(\overrightarrow{\boldsymbol{\varphi}}^{\prime}\right)$ such that $\overrightarrow{\boldsymbol{\varphi}} \subseteq \overrightarrow{\boldsymbol{\varphi}}^{\prime}, \boldsymbol{q}^{\prime} \leqslant \boldsymbol{q}$, and $\boldsymbol{q}^{\prime}$ forc $\overrightarrow{\boldsymbol{\varphi}}^{\prime} \varphi^{-}$. But then $\overrightarrow{\boldsymbol{\pi}} \subseteq \overrightarrow{\boldsymbol{\varphi}}^{\prime}$ and $\boldsymbol{q}^{\prime} \leqslant \boldsymbol{p}$, hence $\boldsymbol{p} \operatorname{forc}_{\vec{\pi}} \varphi$ fails by $3^{\circ}$, which is a contradiction.

The additional result for wforc and $\mathscr{L}(\Sigma+\Pi)_{1}^{1}$ formulas is entirely similar to the induction step $\forall$ as just above.

If $K$ is one of the classes $\mathscr{L}(\Sigma+\Pi)_{1}^{1}, \mathscr{L} \Sigma_{n}^{1}, \mathscr{L} \Pi_{n}^{1}(n \geq 2)$, then let FORC $[K]$ consist of all triples $\langle\overrightarrow{\boldsymbol{\pi}}, \boldsymbol{p}, \varphi\rangle$ such that $\overrightarrow{\boldsymbol{\pi}} \in \mathbf{\mathbf { s M F }}, \boldsymbol{p} \in \mathbf{M T}(\overrightarrow{\boldsymbol{\pi}}), \varphi$ is a closed $\mathscr{L}$ formula of class $K$, and if $n \geq 2$ and $\varphi \in \mathscr{L} \Sigma_{n}^{1} \cup \mathscr{L} \Pi_{n}^{1}$ then $\overrightarrow{\boldsymbol{\pi}} \in \overrightarrow{\mathbf{s M F}}\left[\vec{\Pi} \|_{<n-2}\right]$, and finally $\boldsymbol{p}$ forc $\overrightarrow{\boldsymbol{\pi}} \varphi$. Then FORC $[K]$ is a subset of HC.

Lemma 26.4 (definability, in $\mathbf{L}$ ). FORC $\left[\mathscr{L}(\Sigma+\Pi)_{1}^{1}\right]$ belongs to $\Delta_{1}^{\mathrm{HC}}$. If $n \geq 2$ then FORC $\left[\mathscr{L} \Sigma_{n}^{1}\right]$ belongs to $\Sigma_{n-1}^{\mathrm{HC}}$ and $\mathbf{F O R C}\left[\mathscr{L} \Pi_{n}^{1}\right]$ belongs to $\Pi_{n-1}^{\mathrm{HC}}$.

Proof. Relations like "being an MSP", "being a formula in $\mathscr{L}(\Sigma+\Pi)_{1}^{1}, \mathscr{L} \Sigma_{n}^{1}$, $\mathscr{L} \Pi_{n}^{1} ", \boldsymbol{p} \in \mathbf{M T}(\overrightarrow{\boldsymbol{\rho}})$, forcing over a CTM, etc. are definable in HC by bounded formulas, hence $\Delta_{1}^{\mathrm{HC}}$. On the top of this, the model $\mathfrak{M}$ can be tied by both $\exists$ and $\forall$ in $1^{\circ}$ a, see Remark 26.1. This wraps up the $\Delta_{1}^{\mathrm{HC}}$ estimation for $\mathscr{L}(\Sigma+\Pi)_{1}^{1}$.

The inductive step by $2^{\circ}$ is quite simple.

Now the step by $3^{\circ}$. Assume that $n \geq 2$, and it is already established that FORC $\left[\mathscr{L} \Sigma_{n}^{1}\right] \in \Sigma_{n-1}^{\mathrm{HC}}$. Then $\langle\overrightarrow{\boldsymbol{\pi}}, \boldsymbol{p}, \varphi\rangle \in \mathbf{F O R C}\left[\mathscr{L} \Pi_{n}^{1}\right]$ iff $\overrightarrow{\boldsymbol{\pi}} \in \overrightarrow{\mathbf{s M F}}\left[\overrightarrow{\mathbb{T}} \|_{<n-2}\right]$, $\boldsymbol{p} \in \mathbf{M T}, \varphi$ is a closed $\mathscr{L} \Pi_{n}^{1}$ formula in $\mathfrak{M}(\overrightarrow{\boldsymbol{\pi}})$, and, by $3^{\circ}$, there exist no triple $\left\langle\overrightarrow{\boldsymbol{\pi}}^{\prime}, \boldsymbol{p}^{\prime}, \psi\right\rangle \in \mathbf{F O R C}\left[\mathscr{L} \Sigma_{n}^{1}\right]$ such that $\overrightarrow{\boldsymbol{\pi}}^{\prime} \in \overrightarrow{\mathbf{s M F}}\left[\overrightarrow{\mathbb{\pi}} \|_{<n-2}\right], \overrightarrow{\boldsymbol{\pi}} \subseteq \overrightarrow{\boldsymbol{\pi}}^{\prime}, \boldsymbol{p}^{\prime} \in \mathbf{M T}\left(\overrightarrow{\boldsymbol{\pi}}^{\prime}\right)$, $\boldsymbol{p}^{\prime} \leqslant \boldsymbol{p}$, and $\psi$ is $\varphi^{-}$. Evaluating the key conjunct $\overrightarrow{\boldsymbol{\pi}}^{\prime} \in \overrightarrow{\mathbf{s M F}}\left[\overrightarrow{\boldsymbol{\Pi}} \|_{<n-2}\right]$ by Lemma 25.2 as $\Delta_{n-1}^{\mathrm{HC}}$, we get a required estimation $\Pi_{n-1}^{\mathrm{HC}}$ of $\mathbf{F O R C}\left[\mathscr{L} \Pi_{n}^{1}\right]$.

\section{Forcing simple formulas}

We still argue in $\mathbf{L}$. The following results are mainly related to the relation forc with respect to formulas in the class $\mathscr{L}(\Sigma+\Pi)_{1}^{1}$.

Lemma 27.1 (in L). Assume that $\overrightarrow{\boldsymbol{\pi}} \in \overrightarrow{\mathbf{s M F}}, \overrightarrow{\boldsymbol{\varphi}} \in \overrightarrow{\mathbf{s M F}} \cup \overrightarrow{\mathbf{s M F}}_{\omega_{1}}, \overrightarrow{\boldsymbol{\pi}} \subseteq \overrightarrow{\boldsymbol{\varphi}}$, $\boldsymbol{p} \in \mathbf{M T}(\overrightarrow{\boldsymbol{\pi}}), \varphi$ is a formula in $\mathscr{L}(\Sigma+\Pi)_{1}^{1}, \mathfrak{N} \models \mathbf{Z F L}^{-}$is a TM containing $\overrightarrow{\boldsymbol{\varphi}}$ and $\varphi$, and $\boldsymbol{p} \operatorname{forc}_{\vec{\pi}} \varphi$. Then $\boldsymbol{p} \mathbf{M T}(\overrightarrow{\boldsymbol{\varphi}})$-forces $\varphi[\underline{G}]$ over $\mathfrak{N}$. 
Proof. By definition there is an ordinal $\vartheta<\operatorname{dom} \overrightarrow{\boldsymbol{\pi}}$, a multitree $\boldsymbol{p}_{0} \in \mathbf{M T}(\overrightarrow{\boldsymbol{\pi}} \uparrow \vartheta)$, and a CTM $\mathfrak{M} \models \mathbf{Z F L}^{-}$containing $\varphi$ and $\overrightarrow{\boldsymbol{\pi}}\left\lceil\vartheta\right.$, such that $\overrightarrow{\boldsymbol{\pi}}\left\lceil\vartheta \subset_{\mathfrak{M}} \overrightarrow{\boldsymbol{\pi}}, \boldsymbol{p} \leqslant \boldsymbol{p}_{0}\right.$, and $\boldsymbol{p}_{0} \mathbf{M T}(\overrightarrow{\boldsymbol{\pi}}\lceil\vartheta)$-forces $\varphi[\underline{G}]$ over $\mathfrak{M}$. We can w.l.o.g. assume that $\mathfrak{M} \subseteq \mathfrak{N}$ (by the same reference to Shoenfield as in Remark 26.1).

Now suppose that $G \subseteq \mathbf{M T}(\overrightarrow{\boldsymbol{\varphi}})$ is a set $\mathbf{M T}(\overrightarrow{\boldsymbol{\varphi}})$-generic over $\mathfrak{N}$ and $\boldsymbol{p} \in G$ - then $\boldsymbol{p}_{0} \in G$, too. We have to prove that $\varphi[G]$ is true in $\mathfrak{N}[G]$.

We claim that the set $G^{\prime}=G \cap \mathbf{M T}(\overrightarrow{\boldsymbol{\pi}}\lceil\vartheta)$ is $\mathbf{M T}(\overrightarrow{\boldsymbol{\pi}}\lceil\vartheta)$-generic over $\mathfrak{M}$. Indeed, let a set $\boldsymbol{D} \in \mathfrak{M}, \boldsymbol{D} \subseteq \mathbf{M T}(\overrightarrow{\boldsymbol{\pi}}\lceil\vartheta)$, be open dense in $\mathbf{M T}(\overrightarrow{\boldsymbol{\pi}}\lceil\vartheta)$. Then, as $\overrightarrow{\boldsymbol{\pi}} \uparrow \vartheta \subset_{\mathfrak{M}} \overrightarrow{\boldsymbol{\varphi}}$ by Corollary $20.2(\mathrm{iv}), \boldsymbol{D}$ is pre-dense in $\mathbf{M T}(\overrightarrow{\boldsymbol{\varphi}})$ by $20.2(\mathrm{v})(\mathrm{c})$, and hence $G \cap \boldsymbol{D} \neq \varnothing$ by the choice of $G$. It follows that $G^{\prime} \cap \boldsymbol{D} \neq \varnothing$.

We claim that $\mathbf{c}[G]=\mathbf{c}\left[G^{\prime}\right]$ for any name $\mathbf{c} \in \mathfrak{M}$, in particular, for any name in $\varphi$. Indeed, as $G^{\prime} \subseteq G$, the otherwise occurs by Definition 13.2 only if for some $n, i$ and $\boldsymbol{q}^{\prime} \in K_{n i}^{\mathbf{c}}$ there is $\boldsymbol{q} \in G$ satisfying $\boldsymbol{q} \leqslant \boldsymbol{q}^{\prime}$, but there is no such $\boldsymbol{q}$ in $G^{\prime}$. Let $\boldsymbol{D}$ consist of all multitrees $\left.\boldsymbol{r} \in \mathbf{M T}(\overrightarrow{\boldsymbol{\pi}}\rceil \vartheta\right)$ either satisfying $\boldsymbol{r} \leqslant \boldsymbol{q}^{\prime}$ or somewhere a.d. with $\boldsymbol{q}^{\prime}$. Then $\boldsymbol{D} \in \mathfrak{M}$ and $\boldsymbol{D}$ is open dense in $\mathbf{M T}(\overrightarrow{\boldsymbol{\pi}} \uparrow \vartheta)$. Therefore $\boldsymbol{D} \cap G^{\prime} \neq \varnothing$ by the above, so let $\boldsymbol{r} \in \boldsymbol{D} \cap G^{\prime}$. If $\boldsymbol{r} \leqslant \boldsymbol{q}^{\prime}$ then we get a contradiction with the choice of $\boldsymbol{q}^{\prime}$. If $\boldsymbol{r}$ is somewhere a.d. with $\boldsymbol{q}^{\prime}$ then we get a contradiction with the choice of $\boldsymbol{q}$ as both $\boldsymbol{q}, \boldsymbol{r}$ belong to the generic filter $G$.

It follows that $\varphi[G]$ coincides with $\varphi\left[G^{\prime}\right]$.

Note also that $\boldsymbol{p}_{0} \in G^{\prime}$. We conclude that $\varphi\left[G^{\prime}\right]$ is true in $\mathfrak{M}\left[G^{\prime}\right]$ as $\boldsymbol{p}_{0}$ forces $\varphi[\underline{G}]$ over $\mathfrak{M}$. The same formula $\varphi[G]$ is true in $\mathfrak{N}[G]$ by Shoenfield.

Lemma 27.2. Let $\overrightarrow{\boldsymbol{\pi}} \in \overrightarrow{\mathbf{s M F}}, \boldsymbol{p} \in \mathbf{M T}(\overrightarrow{\boldsymbol{\pi}}), \varphi$ be a formula in $\mathscr{L}(\Sigma+\Pi)_{1}^{1}$. Then

(i) $\boldsymbol{p} \operatorname{forc}_{\vec{\pi}} \varphi$ and $\boldsymbol{p}$ forc $\overrightarrow{\boldsymbol{\pi}} \neg \varphi$ cannot hold together;

(ii) if $\boldsymbol{p}$ forc $\overrightarrow{\boldsymbol{\pi}} \varphi$ then $\boldsymbol{p}$ wforc $\overrightarrow{\boldsymbol{\pi}} \varphi$;

(iii) if $\boldsymbol{p}$ wforc $\overrightarrow{\boldsymbol{\pi}} \varphi$ then there is a multisequence $\overrightarrow{\boldsymbol{\phi}} \in \overrightarrow{\mathbf{s M F}}$ such that $\overrightarrow{\boldsymbol{\pi}} \subset_{\mathfrak{M}(\overrightarrow{\boldsymbol{\pi}})}$ $\vec{\varphi}$ and $\boldsymbol{p}$ forc $\vec{\varphi} \varphi$;

(iv) $\boldsymbol{p}$ wforc $\overrightarrow{\boldsymbol{\pi}} \varphi$ and $\boldsymbol{p}$ wforc $\overrightarrow{\vec{\pi}} \neg \varphi$ cannot hold together.

Proof. (i) Otherwise $\boldsymbol{p} \mathbf{M T}(\overrightarrow{\boldsymbol{\pi}})$-forces both $\varphi[\underline{G}]$ and $\neg \varphi[\underline{G}]$ over a large enough CTM $\mathfrak{M}$, by Lemma 27.1, which cannot happen.

(ii) Assume that $\boldsymbol{p}$ wforc $\vec{\pi} \varphi$ fails, hence there is a multisequence $\vec{\varphi} \in \overrightarrow{\mathbf{s M F}}$ and a multitree $\boldsymbol{q} \in \mathbf{M T}(\overrightarrow{\boldsymbol{\varphi}})$ such that $\boldsymbol{q} \leqslant \boldsymbol{p}$ and $\boldsymbol{q} \operatorname{forc}_{\overrightarrow{\boldsymbol{\pi}}} \neg \varphi$. But Lemma 26.3 implies $\boldsymbol{q}$ forc $\overrightarrow{\boldsymbol{\pi}} \varphi$, which contradicts to (i).

(iii) Let $\mathfrak{M} \models \mathbf{Z F L}^{-}$be a CTM containing $\varphi$ and $\vec{\pi}$. By Corollary 20.2(ii), there is a multisequence multisequence $\overrightarrow{\boldsymbol{\varphi}} \in \overrightarrow{\mathrm{sMF}}$ with $\overrightarrow{\boldsymbol{\pi}} \subset_{\mathfrak{M}} \overrightarrow{\boldsymbol{\phi}}$. We claim that $\boldsymbol{p} \operatorname{MT}(\overrightarrow{\boldsymbol{\pi}})$-forces $\varphi[\underline{G}]$ over $\mathfrak{M}$ in the usual sense - then by definition $\boldsymbol{p}$ forc $\vec{\phi} \varphi$ (via $\vartheta=\operatorname{dom} \vec{\pi}$ ), and we are done. To prove the claim suppose otherwise. Then there is a multitree $\boldsymbol{q} \in \mathbf{M T}(\overrightarrow{\boldsymbol{\pi}})$ such that $\boldsymbol{q} \leqslant \boldsymbol{p}$ and $\boldsymbol{q} \mathbf{M T}(\overrightarrow{\boldsymbol{\pi}})$-forces $\neg \varphi[\underline{G}]$ over $\mathfrak{M}$, thus $\boldsymbol{q}$ forc $\vec{\phi} \neg \varphi$. But this contradicts to $\boldsymbol{p}$ wforc $\vec{\pi} \varphi$. 
(iv) There is a multisequence $\vec{\varphi} \in \overrightarrow{\mathbf{s M F}}$ by (iii), such that $\vec{\pi} \subset \vec{\varphi}$ and $\boldsymbol{p} \operatorname{forc}_{\vec{\varphi}} \varphi$. Note that still $\boldsymbol{p} \operatorname{wforc} \vec{\varphi} \neg \varphi$ by Lemma 26.3. Extend $\vec{\phi}$ once again, getting a contradiction with (i).

Corollary 27.3. Let $n \geq 2, \overrightarrow{\boldsymbol{\pi}} \in \overrightarrow{\mathbf{s M F}}, \boldsymbol{p} \in \mathbf{M T}(\overrightarrow{\boldsymbol{\pi}}), \varphi$ be a formula in $\mathscr{L} \Sigma_{n}^{1}$. Then $\boldsymbol{p} \operatorname{forc}_{\vec{\pi}} \varphi$ and $\boldsymbol{p}$ forc $\overrightarrow{\boldsymbol{\pi}}^{-}$cannot hold together.

Proof. If $n=1$ then apply Lemma 27.2(i). If $n \geq 2$ then the result immediately follows by definition $\left(3^{\circ}\right.$ in Section 26$)$.

Corollary 27.4 (in L). Assume that $\overrightarrow{\boldsymbol{\pi}} \in \overrightarrow{\mathbf{S M F}}, \boldsymbol{p} \in \mathbf{M T}(\overrightarrow{\boldsymbol{\pi}}), \varphi$ is a formula in $\mathscr{L}(\Sigma+\Pi)_{1}^{1}, \mathfrak{N} \models \mathbf{Z F L} L^{-}$is a TM containing $\vec{\pi}$ and $\varphi$, and $\boldsymbol{p} \operatorname{wforc} \overrightarrow{\boldsymbol{\pi}} \varphi$. Then $\boldsymbol{p} \operatorname{MT}(\overrightarrow{\boldsymbol{\pi}})$-forces $\varphi[\underline{G}]$ over $\mathfrak{N}$ in the usual sense.

This looks like the case $\overrightarrow{\boldsymbol{\rho}}=\overrightarrow{\boldsymbol{\pi}}$ in Lemma 27.1, but forc is weakened to wforc, and $\varphi \in \mathfrak{M}$ (automatic in Lemma 27.1) is added, in the premise.

Proof. Otherwise there is a multitree $\boldsymbol{q} \in \mathbf{M T}(\overrightarrow{\boldsymbol{\pi}}), \boldsymbol{q} \leqslant \boldsymbol{p}$, that $\mathbf{M T}(\overrightarrow{\boldsymbol{\pi}})$-forces $\neg \varphi[\underline{G}]$ over $\mathfrak{N}$. On the other hand, by Lemma 27.2(iii), there is a multisequence $\overrightarrow{\boldsymbol{\phi}} \in \overrightarrow{\mathrm{sMF}}$ such that $\overrightarrow{\boldsymbol{\pi}} \subset_{\mathfrak{M}(\overrightarrow{\boldsymbol{\pi}})} \vec{\phi}$ and $\boldsymbol{p} \operatorname{forc}_{\vec{\phi}} \varphi$, hence, $\boldsymbol{q} \operatorname{forc}_{\vec{\phi}} \varphi$ by Lemma 26.3. However we have $\boldsymbol{q}$ forc $\vec{\phi} \neg \varphi$ by definition $\left(1^{\circ}\right.$ a in Section 26 with $\vartheta=\operatorname{dom} \overrightarrow{\boldsymbol{\pi}})$, which contradicts to Lemma 27.2(i).

\section{Tail invariance}

If $\overrightarrow{\boldsymbol{\pi}}=\left\langle\boldsymbol{\pi}_{\alpha}\right\rangle_{\alpha<\lambda} \in \overrightarrow{\mathbf{s M F}}$ and $\gamma<\lambda=\operatorname{dom} \overrightarrow{\boldsymbol{\pi}}$ then let the $\gamma$-tail $\overrightarrow{\boldsymbol{\pi}} \mid \geq \gamma$ be the restriction $\overrightarrow{\boldsymbol{\pi}} \uparrow[\gamma, \lambda)$ to the ordinal semiinterval $[\gamma, \lambda)=\{\alpha: \gamma \leq \alpha<\bar{\lambda}\}$. Then the multiforcing $\mathbf{M T}\left(\overrightarrow{\boldsymbol{\pi}} \uparrow_{\geq \gamma}\right)=\bigcup_{\gamma \leq \alpha<\lambda}^{\text {cw }} \overrightarrow{\boldsymbol{\pi}}(\alpha)$ is open dense in $\mathbf{M T}(\overrightarrow{\boldsymbol{\pi}})$ by Corollary 20.2(v)(a). Therefore it can be expected that if $\overrightarrow{\boldsymbol{\phi}}$ is another multisequence of the same length $\lambda=\operatorname{dom} \overrightarrow{\boldsymbol{\varphi}}$, and $\left.\overrightarrow{\boldsymbol{\varphi}}\right|_{\geq \gamma}=\left.\overrightarrow{\boldsymbol{\pi}}\right|_{\geq \gamma}$, then the relation $\operatorname{forc} \overrightarrow{\boldsymbol{\pi}}$ coincides with forc $\vec{\rho}$. And indeed this turns out to be the case (almost).

Theorem 28.1. Assume that $\overrightarrow{\boldsymbol{\pi}}, \overrightarrow{\boldsymbol{\varphi}}$ are multisequences in $\overrightarrow{\mathbf{s M F}}, \gamma<\lambda=$ $\operatorname{dom} \overrightarrow{\boldsymbol{\pi}}=\operatorname{dom} \overrightarrow{\boldsymbol{\phi}},\left.\overrightarrow{\boldsymbol{\varphi}}\right|_{\geq \gamma}=\left.\overrightarrow{\boldsymbol{\pi}}\right|_{\geq \gamma}, \boldsymbol{p} \in \mathbf{M T}$, and $\varphi$ is an $\mathscr{L}$-formula. Then

(i) if $\varphi \in \mathscr{L}(\Sigma+\Pi)_{1}^{1}$ then $\boldsymbol{p}$ wforc $\overrightarrow{\boldsymbol{\pi}} \varphi$ iff $\boldsymbol{p}$ wforc $\overrightarrow{\boldsymbol{\rho}} \varphi$;

(ii) if $n \geq 2, \overrightarrow{\boldsymbol{\pi}}, \overrightarrow{\boldsymbol{\phi}} \in \overrightarrow{\mathbf{s M F}}\left[\vec{\Pi} \|_{<n-2}\right]$, and $\varphi \in \mathscr{L} \Pi_{n}^{1} \cup \mathscr{L} \Sigma_{n+1}^{1}$, then $\boldsymbol{p} \operatorname{forc}_{\vec{\pi}} \varphi$ iff $\boldsymbol{p}$ forc $\vec{\varphi} \varphi$.

Proof. (i) Suppose to the contrary that $\boldsymbol{p}$ wforc $\vec{\pi} \varphi$, but $\boldsymbol{p}$ wforc $\vec{\phi} \varphi$ fails, so there is a multisequence $\overrightarrow{\boldsymbol{\varphi}}^{\prime} \in \overrightarrow{\mathbf{s M F}}$ and $\boldsymbol{p}^{\prime} \in \mathbf{M T}\left(\overrightarrow{\boldsymbol{\varphi}}^{\prime}\right)$ such that $\overrightarrow{\boldsymbol{\varphi}} \subset \overrightarrow{\boldsymbol{\varphi}}^{\prime}, \boldsymbol{p}^{\prime} \leqslant \boldsymbol{p}$, and $\boldsymbol{p}^{\prime}$ forc $\overrightarrow{\boldsymbol{\varphi}}^{\prime} \neg \varphi$. Let $\lambda^{\prime}=\operatorname{dom} \overrightarrow{\boldsymbol{\varphi}}^{\prime}$. By Corollary 20.2(v)(a), there is a multitree $\boldsymbol{r} \in \mathbf{M T}\left(\left.\overrightarrow{\boldsymbol{\varphi}}^{\prime}\right|_{\geq \gamma}\right), \boldsymbol{r} \leqslant \boldsymbol{p}^{\prime}$. Then still $\boldsymbol{r} \leqslant \boldsymbol{p}$ and $\boldsymbol{r}$ forc $\overrightarrow{\boldsymbol{\phi}}^{\prime} \neg \varphi$, by Lemma 26.3. 
Define a multisequence $\overrightarrow{\boldsymbol{\pi}}^{\prime}$ so that $\operatorname{dom} \overrightarrow{\boldsymbol{\pi}}^{\prime}=\lambda^{\prime}=\operatorname{dom} \overrightarrow{\boldsymbol{\varphi}}^{\prime}, \overrightarrow{\boldsymbol{\pi}} \subseteq \overrightarrow{\boldsymbol{\pi}}^{\prime}$, and $\left.\overrightarrow{\boldsymbol{\pi}}^{\prime}\right|_{\geq \lambda}=\left.\overrightarrow{\boldsymbol{\varphi}}^{\prime}\right|_{\geq \lambda}$. Then $\boldsymbol{r} \in \mathbf{M T}\left(\overrightarrow{\boldsymbol{\pi}}^{\prime}\right)$, and $\boldsymbol{r}$ wforc $\overrightarrow{\boldsymbol{\pi}}^{\prime} \varphi$ by Lemma 26.3.

Consider any $\mathrm{CTM} \mathfrak{N} \models \mathbf{Z F L}^{-}$containing $\varphi, \overrightarrow{\boldsymbol{\pi}}^{\prime}, \overrightarrow{\boldsymbol{\varphi}}^{\prime}$. Then, by Corollary 27.1, one and the same multitree $\boldsymbol{r} \mathbf{M T}\left(\overrightarrow{\boldsymbol{\pi}}^{\prime}\right)$-forces $\varphi[\underline{G}]$ but $\mathbf{M T}\left(\overrightarrow{\boldsymbol{\varphi}}^{\prime}\right)$-forces $\neg \varphi[\underline{G}]$ over $\mathfrak{N}$. But this contradicts to the fact that the forcing notions $\mathbf{M T}\left(\vec{\pi}^{\prime}\right)$, $\operatorname{MT}\left(\overrightarrow{\boldsymbol{\varphi}}^{\prime}\right)$ contain one and the same dense set $\mathbf{M T}\left(\overrightarrow{\boldsymbol{\pi}}^{\prime} \uparrow_{\geq \lambda}\right)=\mathbf{M T}\left(\overrightarrow{\boldsymbol{\varphi}}^{\prime} \uparrow_{\geq \lambda}\right)$.

(ii) Consider first the $\mathscr{L} \Pi_{2}^{1}$ case. Assume that $\varphi(x)$ is a $\mathscr{L} \Sigma_{1}^{1}$ formula, $\boldsymbol{p} \operatorname{forc}_{\vec{\pi}} \forall x \varphi(x)$, but to the contrary $\boldsymbol{p}$ forc $_{\vec{\phi}} \forall x \varphi(x)$ fails. Thus there is a multisequence $\overrightarrow{\boldsymbol{\varphi}}^{\prime} \in \overrightarrow{\mathbf{s M F}}$ and a multitree $\boldsymbol{p}^{\prime} \in \mathbf{M T}\left(\overrightarrow{\boldsymbol{\varphi}}^{\prime}\right)$ such that $\overrightarrow{\boldsymbol{\varphi}} \subseteq \overrightarrow{\boldsymbol{\varphi}}^{\prime}$, $\boldsymbol{p}^{\prime} \leqslant \boldsymbol{p}$, and $\boldsymbol{p}^{\prime}$ forc $\overrightarrow{\boldsymbol{\varphi}}^{\prime} \exists x \varphi^{-}(x)$. By definition there is a small real name $\mathbf{c}$ such that $\boldsymbol{p}^{\prime}$ forc $\overrightarrow{\boldsymbol{\varphi}}^{\prime} \varphi^{-}(\mathbf{c})$. There is a multitree $\boldsymbol{r} \in \mathbf{M T}\left(\left.\overrightarrow{\boldsymbol{\varphi}}^{\prime}\right|_{\geq \gamma}\right), \boldsymbol{r} \leqslant \boldsymbol{p}^{\prime}$. Then still $\boldsymbol{r} \leqslant \boldsymbol{p}$ and $\boldsymbol{r}$ forc $\vec{q}^{\prime} \varphi^{-}(\mathbf{c})$. As above there is a multisequence $\overrightarrow{\boldsymbol{\pi}}^{\prime}$ such that $\operatorname{dom} \overrightarrow{\boldsymbol{\pi}}^{\prime}=\lambda^{\prime}=\operatorname{dom} \overrightarrow{\boldsymbol{\varphi}}^{\prime}, \overrightarrow{\boldsymbol{\pi}} \subseteq \overrightarrow{\boldsymbol{\pi}}^{\prime}$, and $\overrightarrow{\boldsymbol{\pi}}^{\prime} \uparrow_{\geq \lambda}=\left.\overrightarrow{\boldsymbol{\varphi}}^{\prime}\right|_{\geq \lambda}$. Then $\boldsymbol{r} \in$ $\operatorname{MT}\left(\overrightarrow{\boldsymbol{\pi}}^{\prime}\right)$ and $\boldsymbol{r} \operatorname{wforc}_{\overrightarrow{\boldsymbol{\pi}}^{\prime}} \varphi^{-}(\mathbf{c})$ by the inductive hypothesis. By Lemma 27.2, there is a multisequence $\overrightarrow{\boldsymbol{\sigma}}$ such that $\overrightarrow{\boldsymbol{\pi}}^{\prime} \subseteq \overrightarrow{\boldsymbol{\sigma}}$ and $\boldsymbol{r}$ wforc $\overrightarrow{\boldsymbol{\sigma}} \varphi^{-}(\mathbf{c})$, hence, $\boldsymbol{r}$ wforc $\overrightarrow{\boldsymbol{\sigma}} \exists x \varphi^{-}(x)$. But this contradicts to $\boldsymbol{p}$ forc $\overrightarrow{\boldsymbol{\pi}} \forall x \varphi(x)$, since $\boldsymbol{r} \leqslant \boldsymbol{p}$.

To carry out the step $\mathscr{L} \Pi_{n}^{1} \rightarrow \mathscr{L} \Sigma_{n+1}^{1}, n \geq 2$, let $\varphi(x)$ be a formula in $\mathscr{L} \Pi_{n}^{1}$. Assume that $\boldsymbol{p}$ forc $_{\vec{\pi}} \exists x \varphi(x)$. By definition (see $2^{\circ}$ in Section 26), there is a small real name $\mathbf{c}$ such that $\boldsymbol{p}$ forc $\overrightarrow{\boldsymbol{\pi}} \varphi(\mathbf{c})$. Then we have $\boldsymbol{p}$ forc $\overrightarrow{\boldsymbol{\phi}} \varphi(\mathbf{c})$ by the inductive assumption, thus $\boldsymbol{p}$ forc $\vec{\phi} \exists x \psi(x)$.

To carry out the step $\mathscr{L} \Sigma_{n}^{1} \rightarrow \mathscr{L} \Pi_{n}^{1}, n \geq 3$, assume that $\varphi$ is a $\mathscr{L} \Pi_{n}^{1}$ formula, $\boldsymbol{p}$ forc $\overrightarrow{\boldsymbol{\pi}} \varphi$, but to the contrary $\boldsymbol{p}$ forc $\vec{\phi} \varphi$ fails. Then by $3^{\circ}$ of Section 26, as $\overrightarrow{\boldsymbol{\phi}} \in \overrightarrow{\mathbf{s M F}}\left[\overrightarrow{\mathbb{\pi}} \|_{<n-2}\right]$, there is a multisequence $\overrightarrow{\boldsymbol{\varphi}}^{\prime} \in \overrightarrow{\mathbf{s M F}}\left[\overrightarrow{\mathbb{\Pi}} \|_{<n-2}\right]$ and a multitree $\boldsymbol{p}^{\prime} \in \mathbf{M T}\left(\overrightarrow{\boldsymbol{\varphi}}^{\prime}\right)$ such that $\overrightarrow{\boldsymbol{\phi}} \subseteq \overrightarrow{\boldsymbol{\varphi}}^{\prime}, \boldsymbol{p}^{\prime} \leqslant \boldsymbol{p}$, and $\boldsymbol{p}^{\prime}$ forc $\overrightarrow{\boldsymbol{\phi}}^{\prime} \varphi^{-}$. By Corollary 20.2(v)(a), there is a multitree $\boldsymbol{r} \in \mathbf{M T}\left(\overrightarrow{\boldsymbol{\varphi}}^{\prime} \uparrow_{\geq \gamma}\right), \boldsymbol{r} \leqslant \boldsymbol{p}^{\prime}$. Then still $\boldsymbol{r} \leqslant \boldsymbol{p}$ and $\boldsymbol{r}$ forc $\overrightarrow{\boldsymbol{\phi}}^{\prime} \varphi^{-}$. As above in the proof of (i), there is a multisequence $\overrightarrow{\boldsymbol{\pi}}^{\prime}$ such that $\operatorname{dom} \overrightarrow{\boldsymbol{\pi}}^{\prime}=\lambda^{\prime}=\operatorname{dom} \overrightarrow{\boldsymbol{\varphi}}^{\prime}, \overrightarrow{\boldsymbol{\pi}} \subseteq \overrightarrow{\boldsymbol{\pi}}^{\prime}$, and $\overrightarrow{\boldsymbol{\pi}}^{\prime} \uparrow_{\geq \lambda}=\overrightarrow{\boldsymbol{\varphi}}^{\prime} \uparrow_{\geq \lambda}$.

We claim that $\overrightarrow{\boldsymbol{\pi}}^{\prime} \in \overrightarrow{\mathbf{s M F}}\left[\overrightarrow{\mathbb{\pi}} \|_{<n-2}\right]$. Indeed if $\alpha<\operatorname{dom} \overrightarrow{\boldsymbol{\pi}}$ then $\overrightarrow{\boldsymbol{\pi}}^{\prime}(\alpha)=$ $\overrightarrow{\boldsymbol{\pi}}(\alpha) \quad\left(\right.$ as $\left.\overrightarrow{\boldsymbol{\pi}} \subseteq \overrightarrow{\boldsymbol{\pi}}^{\prime}\right)$, hence $\overrightarrow{\boldsymbol{\pi}}^{\prime}(\alpha)\left\|_{<m-2}=\overrightarrow{\boldsymbol{\pi}}(\alpha)\right\|_{<m-2}=\mathbb{q}_{\alpha} \|_{<m-2}$ (as $\overrightarrow{\boldsymbol{\pi}}$ belongs to $\overrightarrow{\mathbf{s M F}}\left[\vec{\pi} \|_{<n-2}\right]$ ). If $\operatorname{dom} \overrightarrow{\boldsymbol{\pi}} \leq \alpha<\operatorname{dom} \overrightarrow{\boldsymbol{\pi}}^{\prime}$ then $\overrightarrow{\boldsymbol{\pi}}^{\prime}(\alpha)=\overrightarrow{\boldsymbol{\varphi}}^{\prime}(\alpha)$ (as $\overrightarrow{\boldsymbol{\pi}}^{\prime} \uparrow_{\geq \lambda}=\left.\overrightarrow{\boldsymbol{\varphi}}^{\prime}\right|_{\geq \lambda}$ ), hence $\overrightarrow{\boldsymbol{\pi}}^{\prime}(\alpha)\left\|_{<m-2}=\overrightarrow{\boldsymbol{\varphi}}^{\prime}(\alpha)\right\|_{<m-2}=\mathbb{q}_{\alpha} \|_{<m-2}$ (as $\overrightarrow{\boldsymbol{\varphi}}^{\prime}$ belongs to $\left.\overrightarrow{\mathbf{s M F}}\left[\overrightarrow{\mathbb{\pi}} \|_{<n-2}\right]\right)$. Thus $\overrightarrow{\boldsymbol{\pi}}^{\prime}(\alpha)\left\|_{<m-2}=\mathbb{\square}_{\alpha}\right\|_{<m-2}$ for all $\alpha$, meaning that $\overrightarrow{\boldsymbol{\pi}}^{\prime}\left\|_{<m-2} \subset \overrightarrow{\mathbb{\Pi}}\right\|_{<m-2}$ and $\overrightarrow{\boldsymbol{\pi}}^{\prime} \in \overrightarrow{\mathbf{s M F}}\left[\overrightarrow{\mathbb{\Pi}} \|_{<n-2}\right]$. To conclude, $\overrightarrow{\boldsymbol{\pi}}^{\prime} \in$ $\overrightarrow{\mathbf{s M F}}\left[\overrightarrow{\mathbb{\pi}} \Uparrow_{<n-2}\right], \overrightarrow{\boldsymbol{\pi}} \subseteq \overrightarrow{\boldsymbol{\pi}}^{\prime}, \boldsymbol{r} \in \mathbf{M T}\left(\overrightarrow{\boldsymbol{\pi}}^{\prime} \uparrow_{\geq \gamma}\right), \boldsymbol{r} \leqslant \boldsymbol{p}$, and also $\boldsymbol{r}$ forc $\overrightarrow{\boldsymbol{\pi}}^{\prime} \varphi^{-}$by the inductive hypothesis. But this contradicts to the assumption $\boldsymbol{p} \operatorname{forc}_{\vec{\pi}} \varphi$.

\section{Permutations}

Still arguing in $\mathbf{L}$, we let PERM be the set of all bijections $\boldsymbol{h}: \omega_{1} \times \omega \stackrel{\text { onto }}{\longrightarrow}$ $\omega_{1} \times \omega$, such that the kernel $|\boldsymbol{h}|=\{\langle\xi, k\rangle: \boldsymbol{h}(\xi, k) \neq\langle\xi, k\rangle\}$ is at most countable. 
Elements of PERM will be called permutations. If $m<\omega$ then let $\mathrm{PERM}_{m}$ consist of those permutations $\boldsymbol{h} \in \mathrm{PERM}$ satisfying $|\boldsymbol{h}| \subseteq \omega_{1} \times\left(\omega_{1} \backslash m\right)$. In other words, if $\boldsymbol{h} \in \mathrm{PERM}_{m}$ and $\xi<\omega_{1}, k<m$, then $\boldsymbol{h}(\xi, k)=\langle\xi, k\rangle$.

Let $\boldsymbol{h} \in$ PERM. We extend the action of $\boldsymbol{h}$ as follows.

- if $\boldsymbol{p}$ is a multitree then $\boldsymbol{h} \boldsymbol{p}$ is a multitree, $|\boldsymbol{h} \boldsymbol{p}|=\boldsymbol{h} " \boldsymbol{p}=\{\boldsymbol{h}(\xi, k):\langle\xi, k\rangle \in$ $|\boldsymbol{p}|\}$, and $(\boldsymbol{h} \boldsymbol{p})(\boldsymbol{h}(\xi, k))=\boldsymbol{p}(\xi, k)$ whenever $\langle\xi, k\rangle \in|\boldsymbol{p}|$, in other words, $\boldsymbol{h} \boldsymbol{p}$ coincides with the superposition $\boldsymbol{p} \circ\left(\boldsymbol{h}^{-1}\right)$;

- if $\boldsymbol{\pi} \in \mathbf{M T}$ is a multiforcing then $\boldsymbol{h} \cdot \boldsymbol{\pi}=\boldsymbol{\pi} \circ\left(\boldsymbol{h}^{-1}\right)$ is a multiforcing, $|\boldsymbol{h} \cdot \boldsymbol{\pi}|=\boldsymbol{h} " \boldsymbol{\pi}$ and $(\boldsymbol{h} \cdot \boldsymbol{\pi})(\boldsymbol{h}(\xi, k))=\boldsymbol{\pi}(\xi, k)$ whenever $\langle\xi, k\rangle \in|\boldsymbol{\pi}|$;

- if $\mathbf{c} \subseteq \mathbf{M T} \times(\omega \times \omega)$ is a real name, then put $\boldsymbol{h} \mathbf{c}=\{\langle\boldsymbol{h} \boldsymbol{p}, n, i\rangle:\langle\boldsymbol{p}, n, i\rangle \in \mathbf{c}\}$, thus easily $\boldsymbol{h c}$ is a real name as well;

- if $\overrightarrow{\boldsymbol{\pi}}=\left\langle\boldsymbol{\pi}_{\alpha}\right\rangle_{\alpha<\kappa}$ is a multisequence, then $\boldsymbol{h} \overrightarrow{\boldsymbol{\pi}}=\left\langle\boldsymbol{h} \cdot \boldsymbol{\pi}_{\alpha}\right\rangle_{\alpha<\kappa}$, still a multisequence.

- if $\varphi:=\varphi\left(\mathbf{c}_{1}, \ldots, \mathbf{c}_{n}\right)$ is a $\mathscr{L}$-formula (with all names explicitly indicated), then $\boldsymbol{h} \varphi$ is $\varphi\left(\boldsymbol{h} \mathbf{c}_{1}, \ldots, \boldsymbol{h} \mathbf{c}_{n}\right)$.

Many notions and relations defined above are clearly PERM-invariant, e.g., $\boldsymbol{p} \in \mathbf{M T}(\boldsymbol{\pi})$ iff $\boldsymbol{h} \boldsymbol{p} \in \mathbf{M T}(\boldsymbol{h} \cdot \boldsymbol{\pi}), \boldsymbol{\pi} \sqsubset \boldsymbol{\varphi}$ iff $\boldsymbol{h} \cdot \boldsymbol{\pi} \sqsubset \boldsymbol{h} \cdot \boldsymbol{\varphi}$, et cetera. The invariance persists even with respect to the relation forc, at least to some extent.

Theorem 29.1. Assume that $\overrightarrow{\boldsymbol{\pi}} \in \overrightarrow{\mathbf{S M F}}, \boldsymbol{p} \in \mathbf{M T}(\overrightarrow{\boldsymbol{\pi}}), \varphi$ is an $\mathscr{L}$-formula, and $\boldsymbol{h} \in$ PERM. Then

(i) if $\varphi$ belongs to $\mathscr{L}(\Sigma+\Pi)_{1}^{1}$ and $\boldsymbol{p} \operatorname{forc}_{\vec{\pi}} \varphi$, then $(\boldsymbol{h p}) \operatorname{wforc}_{\boldsymbol{h} \overrightarrow{\boldsymbol{\pi}}}(\boldsymbol{h} \varphi)$;

(ii) if $n \geq 2, \boldsymbol{h} \in \mathrm{PERM}_{n-2}$, and $\varphi$ belongs to $\mathscr{L} \Pi_{n}^{1} \cup \mathscr{L} \Sigma_{n+1}^{1}$, then $\boldsymbol{p}$ forc $\overrightarrow{\boldsymbol{\pi}} \varphi \varphi$ iff $(\boldsymbol{h} p) \operatorname{forc}_{\boldsymbol{h} \overrightarrow{\boldsymbol{\pi}}}(\boldsymbol{h} \varphi)$.

Proof. Let $\overrightarrow{\boldsymbol{\varphi}}=\boldsymbol{h} \overrightarrow{\boldsymbol{\pi}}, \boldsymbol{q}=\boldsymbol{h} \boldsymbol{p}, \psi:=\boldsymbol{h} \varphi$.

(i) Suppose to the contrary that $\boldsymbol{p}$ wforc $\vec{\pi} \varphi$, but $\boldsymbol{q}$ wforc $\overrightarrow{\boldsymbol{q}} \psi$ fails, so that there is a multisequence $\overrightarrow{\boldsymbol{\varphi}}^{\prime} \in \overrightarrow{\mathbf{s M F}}$ and $\boldsymbol{q}^{\prime} \in \mathbf{M T}\left(\overrightarrow{\boldsymbol{\varphi}}^{\prime}\right)$ such that $\overrightarrow{\boldsymbol{\varphi}} \subset \overrightarrow{\boldsymbol{\varphi}}^{\prime}, \boldsymbol{q}^{\prime} \leqslant \boldsymbol{q}$, and $\boldsymbol{q}^{\prime}$ forc $\vec{\phi}^{\prime} \neg \psi$. The multisequence $\overrightarrow{\boldsymbol{\pi}}^{\prime}=\boldsymbol{h}^{-1} \overrightarrow{\boldsymbol{\varphi}}^{\prime}$ then satisfies $\overrightarrow{\boldsymbol{\pi}} \subset \overrightarrow{\boldsymbol{\pi}}^{\prime}$, and the multitree $\boldsymbol{p}^{\prime}=\boldsymbol{h}^{-1} \boldsymbol{q}^{\prime}$ belongs to $\mathbf{M T}\left(\overrightarrow{\boldsymbol{\pi}}^{\prime}\right)$ and $\boldsymbol{p}^{\prime} \leqslant \boldsymbol{p}$, hence we have $\boldsymbol{p}^{\prime}$ wforc $\overrightarrow{\boldsymbol{\pi}}^{\prime} \varphi$ by Lemma 26.3.

Now let $\mathfrak{M} \models \mathbf{Z F L}^{-}$be an arbitrary CTM containing $\overrightarrow{\boldsymbol{\pi}}^{\prime}, \overrightarrow{\boldsymbol{\varphi}}^{\prime}, \varphi, \psi, \boldsymbol{h} \uparrow|\boldsymbol{h}|$. Then, by Corollary $27.4, \boldsymbol{p}^{\prime} \mathbf{M T}\left(\overrightarrow{\boldsymbol{\pi}}^{\prime}\right)$-forces $\varphi[\underline{G}]$, but $\boldsymbol{q}^{\prime} \mathbf{M T}\left(\overrightarrow{\boldsymbol{\varphi}}^{\prime}\right)$-forces $\psi[\underline{G}]$, over $\mathfrak{M}$. However the sets $\mathbf{M T}\left(\overrightarrow{\boldsymbol{\pi}}^{\prime}\right), \mathbf{M T}\left(\overrightarrow{\boldsymbol{\varphi}}^{\prime}\right)$ belong to the same model $\mathfrak{M}$, where they are order-isomorphic via the isomorphism induced by $\boldsymbol{h}\lceil|\boldsymbol{h}|$. Therefore, and since $\boldsymbol{q}=\boldsymbol{h} \boldsymbol{p}$ and $\psi=\boldsymbol{h} \varphi$, it cannot happen that both $\boldsymbol{p} \mathbf{M T}\left(\overrightarrow{\boldsymbol{\pi}}^{\prime}\right)$-forces $\varphi[\underline{G}]$ and $\boldsymbol{q} \mathbf{M T}\left(\overrightarrow{\boldsymbol{\varphi}}^{\prime}\right)$-forces $\neg \psi[\underline{G}]$. But this contradicts to the above. 
(ii) Consider first the $\mathscr{L} \Pi_{2}^{1}$ case. Assume that $\varphi(x)$ is a $\mathscr{L} \Sigma_{1}^{1}$ formula, $\psi(x):=\boldsymbol{h} \varphi(x), \boldsymbol{p}$ forc $_{\vec{\pi}} \forall x \varphi(x)$, but to the contrary $\boldsymbol{q}$ forc $_{\vec{\rho}} \forall x \psi(x)$ fails. Thus there is a multisequence $\overrightarrow{\boldsymbol{\varphi}}^{\prime} \in \overrightarrow{\mathbf{s M F}}$ and a multitree $\boldsymbol{q}^{\prime} \in \mathbf{M T}\left(\overrightarrow{\boldsymbol{\varphi}}^{\prime}\right)$ such that $\overrightarrow{\boldsymbol{\varphi}} \subset \overrightarrow{\boldsymbol{\varphi}}^{\prime}, \boldsymbol{q}^{\prime} \leqslant \boldsymbol{q}$, and $\boldsymbol{q}^{\prime}$ forc $\overrightarrow{\boldsymbol{\phi}}^{\prime} \exists x \psi^{-}(x)$. By definition there is a small real name $\mathbf{d}$ such that $\boldsymbol{q}^{\prime}$ forc $\overrightarrow{\boldsymbol{\varphi}}^{\prime} \psi^{-}(\mathbf{d})$. The multisequence $\overrightarrow{\boldsymbol{\pi}}^{\prime}=\boldsymbol{h}^{-1} \overrightarrow{\boldsymbol{\varphi}}^{\prime}$ then satisfies $\overrightarrow{\boldsymbol{\pi}} \subset \overrightarrow{\boldsymbol{\varphi}}$, the multitree $\boldsymbol{p}^{\prime}=\boldsymbol{h}^{-1} \boldsymbol{q}^{\prime}$ belongs to $\mathbf{M T}\left(\overrightarrow{\boldsymbol{\pi}}^{\prime}\right)$ and $\boldsymbol{p}^{\prime} \leqslant \boldsymbol{p}$, $\mathbf{c}=\boldsymbol{h}^{-1} \mathbf{d}$ is a small real name, and we have $\boldsymbol{p}^{\prime}$ wforc $\vec{\pi}^{\prime} \varphi^{-}(\mathbf{c})$ by (i). Then by Lemma 27.2 there is a longer multisequence $\overrightarrow{\boldsymbol{\sigma}} \in \overrightarrow{\mathbf{s M F}}$ satisfying $\overrightarrow{\boldsymbol{\pi}}^{\prime} \subset \overrightarrow{\boldsymbol{\sigma}}$ and $\boldsymbol{p}^{\prime} \operatorname{forc}_{\overrightarrow{\boldsymbol{\sigma}}} \varphi^{-}(\mathbf{c})$, that is, we have $\boldsymbol{p}^{\prime}$ forc $_{\overrightarrow{\boldsymbol{\sigma}}} \exists x \varphi^{-}(x)$. But by definition ( $3^{\circ}$ in Section 26) this contradicts to the assumption $\boldsymbol{p}$ forc $\overrightarrow{\boldsymbol{\pi}} \forall x \varphi(x)$.

To carry out the step $\mathscr{L} \Pi_{n}^{1} \rightarrow \mathscr{L} \Sigma_{n+1}^{1}, n \geq 2$, let $\varphi(x)$ be a formula in $\mathscr{L} \Pi_{n}^{1}, \psi(x):=\boldsymbol{h} \varphi(x)$, and $\boldsymbol{h} \in \mathrm{PERM}_{n-2}$. Assume that $\boldsymbol{p} \operatorname{forc}_{\overrightarrow{\boldsymbol{\pi}}} \exists x \varphi(x)$. By definition (see $2^{\circ}$ in Section 26), there is a small real name c such that $\boldsymbol{p} \operatorname{forc}_{\vec{\pi}} \varphi(\mathbf{c})$. Then we have $\boldsymbol{q}$ forc $\overrightarrow{\boldsymbol{q}} \psi(\mathbf{d})$ by inductive assumption, where $\mathbf{d}=\boldsymbol{h} \mathbf{c}$ is a small real name itself. Thus $\boldsymbol{q}$ forc $\vec{\phi} \exists x \psi(x)$.

To carry out the step $\mathscr{L} \Sigma_{n}^{1} \rightarrow \mathscr{L} \Pi_{n}^{1}, n \geq 3$, let $\varphi$ be a formula in $\mathscr{L} \Pi_{n}^{1}$, and $\boldsymbol{h} \in \mathrm{PERM}_{n-2}$. Let $\boldsymbol{p}$ forc $_{\overrightarrow{\boldsymbol{\pi}}} \varphi$, in particular $\overrightarrow{\boldsymbol{\pi}} \in \overrightarrow{\mathbf{s M F}}\left[\overrightarrow{\mathbb{\pi}} \|_{<n-2}\right]$, but, to the contrary, $\boldsymbol{q}$ forc $_{\vec{\phi}} \psi$ fails, where $\boldsymbol{q}=\boldsymbol{h} \boldsymbol{p}, \overrightarrow{\boldsymbol{\phi}}=\boldsymbol{h} \overrightarrow{\boldsymbol{\pi}}$, and $\psi$ is $\boldsymbol{h} \varphi$, as above.

Then in our assumptions, $\overrightarrow{\boldsymbol{\phi}}\left\|_{<m-2}=\overrightarrow{\boldsymbol{\pi}}\right\|_{<m-2}$, hence $\overrightarrow{\boldsymbol{\phi}} \in \overrightarrow{\mathbf{s M F}}\left[\overrightarrow{\mathbb{\pi}} \|_{<n-2}\right]$ as well. Therefore by definition $\left(3^{\circ}\right.$ in Section 26$)$ there is a multisequence $\vec{\phi}^{\prime} \in$ $\overrightarrow{\mathbf{s M F}}\left[\vec{\pi} \|_{<n-2}\right]$ and $\boldsymbol{q}^{\prime} \in \mathbf{M T}\left(\overrightarrow{\boldsymbol{\varphi}}^{\prime}\right)$ such that $\overrightarrow{\boldsymbol{\varphi}} \subseteq \overrightarrow{\boldsymbol{\varphi}}^{\prime}, \boldsymbol{q}^{\prime} \leqslant \boldsymbol{q}$, and $\boldsymbol{q}^{\prime}$ forc $\overrightarrow{\boldsymbol{\varphi}}^{\prime} \psi^{-}$.

Now let $\boldsymbol{p}^{\prime}=\boldsymbol{h}^{-1} \boldsymbol{q}^{\prime}$ and $\overrightarrow{\boldsymbol{\pi}}^{\prime}=\boldsymbol{h}^{-1} \overrightarrow{\boldsymbol{\phi}}^{\prime}$, so that $\boldsymbol{p}^{\prime} \leqslant \boldsymbol{p}, \overrightarrow{\boldsymbol{\pi}} \subseteq \overrightarrow{\boldsymbol{\pi}}^{\prime}$, and, that is most important, $\overrightarrow{\boldsymbol{\pi}}^{\prime}$ belongs to $\overrightarrow{\mathbf{s M F}}\left[\overrightarrow{\mathbb{\pi}} \|_{<n-2}\right]$ since so does $\overrightarrow{\boldsymbol{\varphi}}^{\prime}$ and $\boldsymbol{h}^{-1} \in \mathrm{PERM}_{n-2}$. Moreover we have $\boldsymbol{p}^{\prime}$ forc $\overrightarrow{\boldsymbol{\pi}}^{\prime} \varphi^{-}$by inductive assumption. We conclude that $\boldsymbol{p}$ forc $\overrightarrow{\boldsymbol{\pi}} \varphi$ fails, which is a contradiction.

\section{Forcing with subsequences of the key multisequence}

We argue in $\mathbf{L}$. The key multisequence $\vec{\mathbb{}}=\left\langle\square_{\alpha}\right\rangle_{\alpha<\omega_{1}} \in \overrightarrow{\mathbf{s M F}}_{\omega_{1}}$, satisfying (i), (ii), (iii) of Theorem 23.1, was fixed by 23.4, and $\mathbb{P}=\mathbf{M T}(\overrightarrow{\mathbb{\pi}})$ is our forcing notion. If $\gamma<\omega_{1}$ then the subsequence $\overrightarrow{\mathbb{\Pi}} \mid \gamma$ belongs to $\overrightarrow{\mathbf{s M F}}\left[\vec{\pi} \|_{<m}\right], \forall m$.

Definition 30.1. We write $\boldsymbol{p} \operatorname{forc}_{\alpha} \varphi$ instead of $\boldsymbol{p} \operatorname{forc}_{\vec{\pi}\lceil\alpha} \varphi$, for the sake of brevity. Let $\boldsymbol{p}$ forc $\varphi$ mean: $\boldsymbol{p}$ forc $_{\alpha} \varphi$ for some $\alpha<\omega_{1}$.

Lemma 30.2 (in $\mathbf{L}$ ). Assume that $\boldsymbol{p} \in \mathbb{P}, \alpha<\omega_{1}$, and $\boldsymbol{p}$ forc $_{\alpha} \varphi$. Then:

(i) if $\alpha \leq \beta<\omega_{1}, \boldsymbol{q} \in \mathbb{P}_{<\beta}=\mathbf{M T}(\overrightarrow{\mathbb{T}} \uparrow \beta)$, and $\boldsymbol{q} \leqslant \boldsymbol{p}$, then $\boldsymbol{q} \operatorname{forc}_{\beta} \varphi$;

(ii) if $\boldsymbol{q} \in \mathbb{P}, \boldsymbol{q} \leqslant \boldsymbol{p}$, then $\boldsymbol{q}$ forc $_{\beta} \varphi$ for some $\beta$; $\alpha \leq \beta<\omega_{1}$;

(iii) if $\boldsymbol{q} \in \mathbb{P}$ and $\boldsymbol{q}$ forc $\varphi^{-}$then $\boldsymbol{p}, \boldsymbol{q}$ are somewhere almost disjoint; 
(iv) therefore, 1st, if $\boldsymbol{p}, \boldsymbol{q} \in \mathbb{P}, \boldsymbol{q} \leqslant \boldsymbol{p}$, and $\boldsymbol{p}$ forc $\varphi$ then $\boldsymbol{q}$ forc $\varphi$, and $2 n d$, $\boldsymbol{p}$ forc $\varphi, \boldsymbol{p}$ forc $\varphi^{-}$cannot hold together.

Proof. To prove (i) apply Lemma 26.3. To prove (ii) let $\beta$ satisfy $\alpha<\beta<\omega_{1}$ and $\boldsymbol{q} \in \mathbf{M T}(\overrightarrow{\mathbb{T}} \uparrow \beta)$, and apply (i). Finally to prove (iii) note that $\boldsymbol{p}, \boldsymbol{q}$ have to be incompatible in $\mathbb{P}$, as otherwise (i) leads to contradiction, but the incompatibility in $\mathbb{P}$ implies being somewhere a.d. by Corollary 7.1 .

Now we are going to prove that the auxiliary relation forc essentially coincides with the usual $\mathbb{P}$-forcing relation over $\mathbf{L}$.

Lemma 30.3. If $n<\omega, \varphi$ is a closed formula as in 26.2, and $\boldsymbol{p} \in \mathbb{P}$, then $\boldsymbol{p}$ $\mathbb{P}$-forces $\varphi[\underline{G}]$ over $\mathbf{L}$ in the usual sense if and only if $\boldsymbol{p}$ forc $\varphi$.

Proof. Let $\Vdash$ denote the usual $\mathbb{P}$-forcing relation over $\mathbf{L}$.

Part 1: $\varphi$ is a formula in $\mathscr{L}(\Sigma+\Pi)_{1}^{1}$. If $\boldsymbol{p}$ forc $\varphi$ then $\boldsymbol{p} \operatorname{forc}_{\vec{\pi} \mid \gamma} \varphi$ for some $\gamma<\omega_{1}$, and then $\boldsymbol{p} \| \varphi[\underline{G}]$ by Lemma 27.1 with $\overrightarrow{\boldsymbol{\phi}}=\overrightarrow{\mathbb{T}}$ and $\mathfrak{N}=\mathbf{L}$.

Suppose now that $\boldsymbol{p} \Vdash \varphi[\underline{G}]$. There is an ordinal $\gamma_{0}<\omega_{1}$ such that $\boldsymbol{p} \in$ $\mathbb{P}_{\gamma_{0}}=\mathbf{M T}\left(\overrightarrow{\mathbb{\pi}}\left\lceil\gamma_{0}\right)\right.$ and $\varphi$ belongs to $\mathfrak{M}\left(\overrightarrow{\mathbb{\pi}}\left\lceil\gamma_{0}\right)\right.$. The set $U$ of all multitrees $\overrightarrow{\boldsymbol{\pi}} \in \overrightarrow{\mathbf{s M F}}$ such that $\gamma_{0}<\operatorname{dom} \overrightarrow{\boldsymbol{\pi}}$ and there is an ordinal $\vartheta, \gamma_{0}<\vartheta<\operatorname{dom} \overrightarrow{\boldsymbol{\pi}}$, such that $\overrightarrow{\boldsymbol{\pi}}\left\lceil\vartheta \subset_{\mathfrak{M}(\overrightarrow{\boldsymbol{\pi}} \mid \vartheta)} \overrightarrow{\boldsymbol{\pi}}\right.$, is dense in $\overrightarrow{\boldsymbol{\pi}}$ by Corollary 20.2(ii), and is $\boldsymbol{\Delta}_{1}^{\mathrm{HC}}$. Therefore by Corollary 23.5 there is an ordinal $\gamma<\omega_{1}$ such that $\vec{\pi}=\overrightarrow{\mathbb{T}} \uparrow \gamma \in U$.

Let this be witnessed by an ordinal $\vartheta$, so that $\gamma_{0}<\vartheta<\gamma=\operatorname{dom} \vec{\pi}$ and $\overrightarrow{\boldsymbol{\pi}}\left\lceil\vartheta \subset_{\mathfrak{M}(\overrightarrow{\boldsymbol{\pi}}\lceil\vartheta)} \overrightarrow{\boldsymbol{\pi}}\right.$. We claim that $\boldsymbol{p} \mathbf{M T}(\overrightarrow{\boldsymbol{\pi}}\lceil\vartheta)$-forces $\varphi[\underline{G}]$ over $\mathfrak{M}(\overrightarrow{\boldsymbol{\pi}}\lceil\vartheta)$ in the usual sense - then by definition $\boldsymbol{p}$ forc $\overrightarrow{\boldsymbol{\pi}} \varphi$, and we are done.

To prove the claim, suppose otherwise. Then there is a multitree $\boldsymbol{q} \in$ $\operatorname{MT}(\overrightarrow{\mathbb{\Pi}}\lceil\vartheta), \boldsymbol{q} \leqslant \boldsymbol{p}$, which $\mathbf{M T}(\overrightarrow{\boldsymbol{\pi}}\lceil\vartheta)$-forces $\neg \varphi[\underline{G}]$ over $\mathfrak{M}(\overrightarrow{\boldsymbol{\pi}}\lceil\vartheta)$. Then by definition we have $\boldsymbol{q}$ forc $\overrightarrow{\boldsymbol{\pi}} \neg \varphi$, hence $\boldsymbol{q}$ forc $\neg \varphi$, which implies $\boldsymbol{q} \Vdash \neg \varphi[\underline{G}]$ (see above), with a contradiction to $\boldsymbol{p} \Vdash \varphi[\underline{G}]$.

Part 2: the step $\mathscr{L} \Pi_{n}^{1} \rightarrow \mathscr{L} \Sigma_{n+1}^{1}(n \geq 1)$. Consider a $\mathscr{L} \Pi_{n}^{1}$ formula $\varphi(x)$. Assume $\boldsymbol{p}$ forc $\exists x \varphi(x)$. By definition there is a small real name $\mathbf{c}$ such that $\boldsymbol{p}$ forc $\varphi(\mathbf{c})$. By inductive hypothesis, $\boldsymbol{p} \Vdash \varphi(c)[\underline{G}]$, that is, $\boldsymbol{p} \Vdash \exists x \varphi(x)[\underline{G}]$. Conversely, assume that $\boldsymbol{p} \Vdash \exists x \varphi(x)[\underline{G}]$. As $\mathbb{P}$ is CCC, there is a small real name c (in $\mathbf{L}$ ) such that $\boldsymbol{p} \Vdash \varphi(\mathbf{c})[\underline{G}]$. We have $\boldsymbol{p}$ forc $\varphi(\mathbf{c})$ by the inductive hypothesis, hence $\boldsymbol{p}$ forc $\exists x \varphi(x)$.

Part 3: the step $\mathscr{L} \Sigma_{n}^{1} \rightarrow \mathscr{L} \Pi_{n}^{1}(n \geq 2)$. Consider a closed $\mathscr{L} \Sigma_{n}^{1}$ formula $\varphi$. Assume that $\boldsymbol{p}$ forc $\varphi^{-}$. By Lemma 30.2(iv), there is no multitree $\boldsymbol{q} \in \mathbb{P}$, $\boldsymbol{q} \leqslant \boldsymbol{p}$, with $\boldsymbol{q}$ forc $\varphi$. This implies $\boldsymbol{p} \Vdash \varphi^{-}$by the inductive hypothesis.

Conversely, suppose that $\boldsymbol{p} \Vdash \varphi^{-}$. There is an ordinal $\gamma_{0}<\omega_{1}$ such that $\boldsymbol{p} \in \mathbb{P}_{\gamma_{0}}=\mathbf{M T}\left(\overrightarrow{\mathbb{T}}\left\lceil\gamma_{0}\right)\right.$ and $\varphi$ belongs to $\mathfrak{M}\left(\overrightarrow{\mathbb{T}} \uparrow \gamma_{0}\right)$. Consider the set $U$ of all multisequences of the form $\overrightarrow{\boldsymbol{\pi}} \|_{\geq n-2}$, where $\overrightarrow{\boldsymbol{\pi}} \in \overrightarrow{\mathbf{s M F}}\left[\overrightarrow{\mathbb{\pi}} \|_{<n-2}\right]$, dom $\overrightarrow{\boldsymbol{\pi}}>\gamma_{0}$, and 
there is a multitree $\boldsymbol{q} \in \mathbf{M T}(\overrightarrow{\boldsymbol{\pi}})$ satisfying $\boldsymbol{q} \leqslant \boldsymbol{p}$ ( $\boldsymbol{q}$ is stronger) and $\boldsymbol{q}$ forc $\overrightarrow{\boldsymbol{\pi}} \varphi$. It follows from Lemma 25.2 and Lemma 26.4 that $U$ belongs to $\boldsymbol{\Sigma}_{n-1}^{\mathrm{HC}}$ (with $\varphi$ and $\boldsymbol{p}_{0}$ as parameters). Therefore by 23.4(ii) there is an ordinal $\gamma<\omega_{1}$ such that the subsequence $\overrightarrow{\mathbb{\pi}} \uparrow \gamma(n-2)$-decides $U$.

Case 1: $(\overrightarrow{\mathbb{\pi}} \uparrow \gamma) \|_{\geq n-2} \in U$. Let this be witnessed by a multisequence $\vec{\pi} \in$ $\overrightarrow{\mathbf{s M F}}\left[\overrightarrow{\mathbb{\Pi}} \|_{<n-2}\right]$ and a multitree $\boldsymbol{q} \in \mathbf{M T}(\overrightarrow{\boldsymbol{\pi}})$, so that in particular $(\overrightarrow{\mathbb{\Pi}} \uparrow \gamma) \|_{\geq n-2}=$ $\vec{\pi} \|_{\geq n-2}$ and $\operatorname{dom} \vec{\pi}=\gamma>\gamma_{0}$. Then by definition (Definition 25.1) we also have $\overrightarrow{\boldsymbol{\pi}} \|_{<n-2}=\left(\overrightarrow{\mathbb{\pi}}\lceil\gamma) \|_{<n-2}\right.$, so that overall $\overrightarrow{\boldsymbol{\pi}}=\vec{\mathbb{}}\lceil\gamma$. Thus $\boldsymbol{q} \in \mathbf{M T}(\overrightarrow{\mathbb{\pi}}\lceil\gamma), \boldsymbol{q} \leqslant \boldsymbol{p}$, and $\boldsymbol{q} \operatorname{forc}_{\vec{\pi} \mid \gamma} \varphi$, that is, $\boldsymbol{q} \Vdash \varphi[\underline{G}]$ by the inductive hypothesis, contrary to the choice of $\boldsymbol{p}$. Therefore Case 1 cannot happen, and we have:

Case 2: negative decision, no multisequence in $U$ extends $\left(\vec{\mathbb{}}\lceil\gamma) \|_{\geq n-2}\right.$. We can assume that $\gamma>\gamma_{0}$. (Otherwise replace $\gamma$ by $\gamma_{0}+1$.) We claim that $\boldsymbol{p}$ forc $_{\gamma} \varphi^{-}$. Indeed otherwise by $3^{\circ}$ there is a multisequence $\overrightarrow{\boldsymbol{\pi}} \in \overrightarrow{\mathbf{s M F}}\left[\overrightarrow{\mathbb{\pi}} \pi_{<n-2}\right]$ and a multitree $\boldsymbol{q} \in \mathbf{M T}(\overrightarrow{\boldsymbol{\pi}})$, such that $\overrightarrow{\mathbb{\pi}} \mid \gamma \subseteq \overrightarrow{\boldsymbol{\pi}}, \boldsymbol{q} \leqslant \boldsymbol{p}$, and $\boldsymbol{q}$ forc $\overrightarrow{\boldsymbol{\phi}} \varphi$. But then $\overrightarrow{\boldsymbol{\pi}}$ and $\boldsymbol{q}$ witness that $\overrightarrow{\boldsymbol{\sigma}}=\overrightarrow{\boldsymbol{\pi}} \|_{\geq n-2}$ belongs to $U$. On the other hand, $\vec{\sigma}$ obviously extends $\vec{\mathbb{}}\left\lceil\gamma \|_{\geq n-2}\right.$, since $\vec{\square}\lceil\gamma \subseteq \vec{\pi}$, contrary to the Case 2 assumption. Thus indeed $\boldsymbol{p}$ forc $\varphi^{-}$, as required.

The next lemma provides a useful strengthening.

Lemma 30.4. If $\Phi$ is a $\Delta_{1}^{\mathrm{HC}}$ collection of closed $\Pi_{n+2}^{1}$ formulas, $\boldsymbol{p}_{0} \in \mathbb{P}$, and $\boldsymbol{p}_{0} \mathbb{P}$-forces $\varphi[\underline{G}]$ over $\mathbf{L}$ for each $\varphi \in \Phi$, then there is an ordinal $\gamma<\omega_{1}$ such that if $\varphi \in \Phi$ then $\boldsymbol{p}_{0}$ forc $\overrightarrow{\mathbb{\pi}} \mid \gamma \varphi$. (Same $\gamma$ for all $\varphi$.)

Proof. Let $U$ consist of all multisequences of the form $\overrightarrow{\boldsymbol{\pi}} \Uparrow_{\geq m}$, where $\overrightarrow{\boldsymbol{\pi}} \in$ $\overrightarrow{\mathbf{S M F}}\left[\overrightarrow{\mathbb{\pi}} \|_{<m}\right]$, and there is a formula $\varphi \in \Phi$ and $\boldsymbol{p} \in \mathbf{M T}(\overrightarrow{\boldsymbol{\pi}})$ such that $\boldsymbol{p} \leqslant \boldsymbol{p}_{0}$ and $\boldsymbol{p}$ forc $_{\vec{\pi}} \varphi^{-}$. It follows from Lemma 25.2 and 26.4 that $U$ is a $\boldsymbol{\Sigma}_{m+1}^{\mathrm{HC}}$ set, so by 23.4(ii) there is an ordinal $\gamma<\omega_{1}$ such that $\vec{\mathbb{}} \uparrow \gamma m$-decides $U$.

Case 1: $\left(\overrightarrow{\mathbb{\pi}}\lceil\gamma) \|_{\geq m} \in U\right.$, that is, the multisequence $\vec{\pi}=\vec{\mathbb{}} \uparrow \gamma$ satisfies the condition that there exist $\varphi \in \Phi$ and a multitree $\boldsymbol{p} \in \overrightarrow{\boldsymbol{\pi}}$ such that $\boldsymbol{p} \leqslant \boldsymbol{p}_{0}$ and $\boldsymbol{p}$ forc $_{\overrightarrow{\boldsymbol{\pi}}} \varphi^{-}$, and hence $\boldsymbol{p} \mathbb{P}$-forces $\varphi^{-}[\underline{G}]$ over $\mathbf{L}$ by Lemma 30.3, contrary to the choice of $\boldsymbol{p}_{0}$. Therefore Case 1 cannot happen, and we have

Case 2: no multisequence in $U$ extends $(\overrightarrow{\mathbb{T} \uparrow} \gamma) \|_{\geq m}$. We can assume that $\gamma>\gamma_{0}$. (Otherwise replace $\gamma$ by $\gamma_{0}+1$.) We claim that $\gamma$ is as required. Indeed otherwise $\boldsymbol{p}_{0}$ forc $\overrightarrow{\mathbb{\pi}} \mid \gamma \varphi$ fails for a formula $\varphi \in \Phi$, thus ( $3^{\circ}$ in Section 26), there is a multisequence $\overrightarrow{\boldsymbol{\pi}} \in \overrightarrow{\mathbf{s M F}}\left[\overrightarrow{\mathbb{\pi}} \|_{<m}\right]$ and $\boldsymbol{p} \in \mathbf{M T}(\overrightarrow{\boldsymbol{\pi}})$ such that $\vec{\mathbb{}}\lceil\gamma \subseteq \overrightarrow{\boldsymbol{\pi}}, \boldsymbol{p} \leqslant$ $\boldsymbol{p}_{0}$, and $\boldsymbol{p}$ forc $\overrightarrow{\boldsymbol{\pi}} \varphi^{-}$. It follows that $\overrightarrow{\boldsymbol{\pi}} \|_{\geq m} \in U$. In addition, $\overrightarrow{\boldsymbol{\pi}} \|_{\geq m}$ extends $\left(\overrightarrow{\mathbb{\nabla}}\lceil\gamma) \|_{\geq m}\right.$ by construction. But this contradicts to the Case 2 assumption. 


\section{The model}

In this conclusive section we gather the results obtained above towards the proof of Theorem 1.1. We begin with the analysis of definability of key generic reals in $\mathbb{P}$-generic extensions of $\mathbf{L}$, which will lead to (I) and (II) of Theorem 1.1. Then we proceed to (III) (elementary equivalence) and (IV) (the non-wellorderability).

\section{Key generic extension and subextensions}

Recall that the key multisequence $\vec{\mathbb{}}=\left\langle\square_{\alpha}\right\rangle_{\alpha<\omega_{1}}$ of small multiforcings $\square_{\alpha}$ is defined in $\mathbf{L}$ by 23.4, $\mathbb{\square}=\bigcup_{\alpha<\omega_{1}}^{\mathrm{cw}}$ is a multiforcing, $|\boldsymbol{\square}|=\omega_{1} \times \omega$ in $\mathbf{L}$, and $\mathbb{P}=\mathbf{M T}(\overrightarrow{\mathbb{T}})=\mathbf{M T}(\mathbb{\square}) \in \mathbf{L}$ is our key forcing notion, equal to the finite-support product $\prod_{\xi<\omega_{1}, k<\omega} \llbracket(\xi, k)$ of perfect-tree forcings $\square(\xi, k)$ in $\mathbf{L}$. See Section 24, where some properties of $\mathbb{P}$ are established, including CCC and definability of the factors $\llbracket(\xi, k)$.

From now on, we'll typically argue in $\mathbf{L}$ and in $\mathbb{P}$-generic extensions of $\mathbf{L}$, so by Lemma 24.6 it will always be true that $\omega_{1}^{\mathbf{L}}=\omega_{1}$. This allows us to still think that $|\square|=\omega_{1} \times \omega\left(\right.$ rather than $\left.\omega_{1}^{\mathbf{L}} \times \omega\right)$.

Recall that $\square \in \mathbf{L}$ and $\mathbb{P}=\mathbf{M T}(\square)$ is a forcing notion in $\mathbf{L}$.

Definition 31.1. Let a set $G \subseteq \mathbb{P}$ be generic over the constructible set universe L. If $\langle\xi, k\rangle \in \omega_{1} \times \omega$ then following Remark 7.3, we

- define $G(\xi, k)=\left\{T_{\xi k}^{\boldsymbol{p}}: \boldsymbol{p} \in G \wedge\langle\xi, k\rangle \in|\boldsymbol{p}|\right\} \subseteq \llbracket(\xi, k) ;$

- let $x_{\xi k}=x_{\xi k}[G] \in 2^{\omega}$ be the only real in $\bigcap_{T \in G(\xi, k)}[T]$.

Thus $\mathbb{P}$ adds an array $X=\left\langle x_{\xi k}\right\rangle_{\langle\xi, k\rangle \in \omega_{1} \times \omega}$ of reals, where each real $x_{\xi k}=$ $x_{\xi k}[G] \in \in 2^{\omega} \cap \mathbf{L}[G]$ is a $\llbracket(\xi, k)$-generic real over $\mathbf{L}$, and $\mathbf{L}[G]=\mathbf{L}[X]$.

Let $G \subseteq \mathbb{P}$ be a set (filter) $\mathbb{P}$-generic over $\mathbf{L}$. If $m<\omega$ then following the notation in Section 21 we define

$$
G \Uparrow_{<m}=G \cap \mathbf{M T} \|_{<m}=\left\{\boldsymbol{p} \|_{<m}: \boldsymbol{p} \in G\right\},
$$

so that the set $G \|_{<m}$ is $\mathbb{P} \|_{<m}$-generic over $\mathbf{L}$, where accordingly

$$
\mathbb{P}\left\|_{<m}=\mathbb{P} \cap \mathbf{M T}\right\|_{<m}=\left\{\boldsymbol{p} \|_{<m}: \boldsymbol{p} \in \mathbb{P}\right\} .
$$

Each subextension $\mathbf{L}\left[G \Uparrow_{<m}\right] \subseteq \mathbf{L}[G]$ coincides with $\mathbf{L}\left[\left\langle x_{\xi k}[G]\right\rangle_{\xi<\omega_{1} \wedge k<m}\right]$. Our goal will be to demonstrate that the model $\mathbf{L}[X]=\mathbf{L}[G]$, along with the system of submodels $\mathbf{L}\left[\left\langle x_{\xi k}[G]\right\rangle_{\xi<\omega_{1} \wedge k<m}\right]$, proves Theorem 1.1. 


\section{Definability of generic reals}

Recall that the factors $\square(\xi, k)$ of the forcing notion $\square$ are defined by $\square(\xi, k)=$ $\bigcup_{\alpha(\xi, k) \leq \alpha<\omega_{1}} \rrbracket_{\alpha}(\xi, k)$, where $\alpha(\xi, k)<\omega_{1}$, the sets $\square_{\alpha}(\xi, k)$ are countable sets of perfect trees, whose definability in $\mathbf{L}$ is determined by Corollary 24.3.

Theorem 32.1. Assume that a set $G \subseteq \mathbb{P}$ is $\mathbb{P}$-generic over $\mathbf{L}, \xi<\omega_{1}, k<\omega$, and $x \in \mathbf{L}[G] \cap \omega^{\omega}$. The following are equivalent:

(1) $x=x_{\xi k}[G]$;

(2) $x$ is $\square(\xi, k)$-generic over $\mathbf{L}$;

(3) $x \in \bigcap_{\alpha(\xi, k) \leq \alpha<\omega_{1}} \bigcup_{T \in \mathbb{\Pi}_{\alpha}(\xi, k)}[T]$.

Proof. (1) $\Longrightarrow(2)$ is a routine (see Remark 7.3). To check $(2) \Longrightarrow(3)$ recall that each set $\mathbb{\square}_{\alpha}(\xi, k)$ is pre-dense in $\llbracket(\xi, k)$ by Lemma $9.2(\mathrm{v})$. It remains to establish $(3) \Longrightarrow(1)$. Suppose towards the contrary that a real $x \in \mathbf{L}[G] \cap 2^{\omega}$ satisfies $(3)$ but $x \neq x_{\xi k}[G]$. By Theorem 18.2(i) there is a true $\mathbb{1}$-real name $\mathbf{c}=\left\langle C_{n i}\right\rangle_{n, i<\omega}$, non-principal over $\mathbb{\square}$ at $\xi, k$ and such that $x=\mathbf{c}[G]$. Being

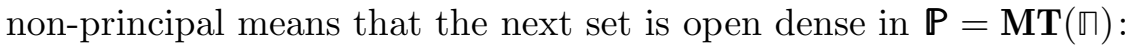

$$
\boldsymbol{D}_{\xi k}^{\prod}(\mathbf{c})=\left\{\boldsymbol{p} \in \mathbb{P}=\mathbf{M T}(\mathbb{\square}): \boldsymbol{p} \text { directly forces } \mathbf{c} \notin\left[T_{\xi k}^{\boldsymbol{p}}\right]\right\} .
$$

And as $\mathbb{P}=\mathbf{M T}(\square)$ is a CCC forcing by Lemma 24.6, we can assume that the name c is small, that is, each set $C_{n i} \subseteq \mathbb{P}$ is countable. Then there is an ordinal $\gamma_{0}<\omega_{1}$ such that $C_{n i} \subseteq \mathbb{P}_{<\gamma_{0}}$ for all $n, i$. Then $\mathbf{c}$ is a true $\mathbb{q}_{<\gamma_{0}}$-real name. Moreover we can assume by Corollary 24.7 that $\boldsymbol{D}_{\xi k}^{\prod}(\mathbf{c}) \cap \mathbb{P}_{<\gamma_{0}}$ is pre-dense in $\mathbb{P}$.

Now consider the set $W$ of all multisequences $\overrightarrow{\boldsymbol{\pi}}=\left\langle\boldsymbol{\pi}_{\alpha}\right\rangle_{\alpha<\operatorname{dom}(\overrightarrow{\boldsymbol{\pi}})} \in \overrightarrow{\mathbf{s M F}}$ such that $\operatorname{dom}(\overrightarrow{\boldsymbol{\pi}})>\gamma_{0}$ and

- either (I) $\vec{\pi} \uparrow \gamma_{0} \not \subset \vec{\pi}$;

- or (II) $\overrightarrow{\mathbb{\pi}} \uparrow \gamma_{0} \subset \overrightarrow{\boldsymbol{\pi}}$ and c is not non-principal over $\boldsymbol{\pi}=\bigcup^{\mathrm{cw}} \overrightarrow{\boldsymbol{\pi}}$ at $\xi, k$;

- or (III) $\overrightarrow{\mathbb{\pi}}\left\lceil\gamma_{0} \subset \overrightarrow{\boldsymbol{\pi}}, \operatorname{dom}(\overrightarrow{\boldsymbol{\pi}})=\delta+1\right.$ is a successor, and $\bigcup_{\alpha<\delta}^{\mathrm{cw}} \boldsymbol{\pi}_{\alpha} \sqsubset_{\xi k}^{\mathbf{c}} \boldsymbol{\pi}_{\delta}$.

We assert that $W$ is dense in $\overrightarrow{\mathbf{S M F}}$ : any multisequence $\overrightarrow{\boldsymbol{\pi}} \in \overrightarrow{\mathbf{s M F}}$ can be extended to some $\overrightarrow{\boldsymbol{\varphi}} \in W$. Indeed first extend $\overrightarrow{\boldsymbol{\pi}}$ by Corollary 20.2 so that is has a length $\operatorname{dom}(\overrightarrow{\boldsymbol{\pi}})=\delta>\gamma_{0}$. If now $\vec{\mathbb{}} \uparrow \gamma_{0} \not \subset \overrightarrow{\boldsymbol{\pi}}$ then immediately $\overrightarrow{\boldsymbol{\pi}} \in W$ via (I), so we assume that $\overrightarrow{\mathbb{\pi}}\left\lceil\gamma_{0} \subset \overrightarrow{\boldsymbol{\pi}}\right.$. We can also assume that $\mathbf{c}$ is non-principal over $\boldsymbol{\pi}=\bigcup^{\mathrm{cw}} \overrightarrow{\boldsymbol{\pi}}$ at $\xi, k$ by similar reasons related to (II). The multisequence $\overrightarrow{\boldsymbol{\pi}}$ can be extended, by Corollary 20.2 , by an extra term $\boldsymbol{\pi}_{\delta}$, so that the extended multisequence $\overrightarrow{\boldsymbol{\pi}}_{+}$satisfies $\overrightarrow{\boldsymbol{\pi}} \subset_{\{\mathbf{c}\}} \overrightarrow{\boldsymbol{\pi}}_{+}$, that is, $\boldsymbol{\pi} \mathbb{L}_{\{\mathbf{c}\}} \boldsymbol{\pi}_{\delta}$. By definition (Definition 19.1) and the nonprincipality of $\mathbf{c}$, we get $\boldsymbol{\pi} \sqsubset_{\xi k}^{\mathbf{c}} \boldsymbol{\pi}_{\delta}$. It follows that $\overrightarrow{\boldsymbol{\pi}}_{+} \in W$ via (III). 
Since $W$ is $\boldsymbol{\Sigma}_{1}^{\mathrm{HC}}$, by Definition 23.4(ii) there is an ordinal $\gamma<\omega_{1}$ such that the multisequence $\vec{\mathbb{}} \mid \gamma 0$-decides $W$. However the negative decision is impossible by the density (see the proof of Lemma 24.2). We conclude that $\overrightarrow{\mathbb{\Pi}}\left\lceil\gamma \in W\right.$; hence, $\gamma>\gamma_{0}$. Option (I) for $\overrightarrow{\boldsymbol{\pi}}=\vec{\mathbb{}}\lceil\gamma$ clearly fails, and (II) fails either because the set $\boldsymbol{D}_{\xi k}^{\mathbb{V}}(\mathbf{c}) \cap \mathbb{P}_{<\gamma_{0}}$ is pre-dense in $\mathbb{P}$ and $\gamma>\gamma_{0}$. Therefore $\vec{\Pi}\left\lceil\gamma\right.$ belongs to $W$ via (III), that is, $\gamma=\delta+1$ and $\rrbracket_{<\delta}=\bigcup_{\alpha<\delta}^{\mathrm{cw}} \square_{\alpha} \sqsubset_{\xi k}^{\mathrm{c}} \rrbracket_{\delta}$. Then $\rrbracket_{<\delta} \sqsubset_{\xi k}^{\mathrm{c}} \mathbb{\square}_{\geq \delta}=\bigcup_{\delta \leq \alpha<\omega_{1}}^{\mathrm{cw}} \rrbracket_{\delta}$ by Lemma 16.3(iii).

Now we make use of Theorem 18.2(ii) with $\boldsymbol{\pi}=\mathbb{\square}_{<\delta}$ and $\boldsymbol{Q}=\mathbb{\square}_{\geq \delta}$; note that $\boldsymbol{\pi} \cup^{\mathrm{cw}} \boldsymbol{\varphi}=\llbracket$. It follows that $x=\mathbf{c}[G] \notin \bigcup_{Q \in \mathbb{M}_{\geq \delta}(\xi, k)}[Q]$, which clearly contradicts to the assumption (3).

Corollary 32.2. Assume that $k<\omega$ and $G \subseteq \mathbb{P}$ is $\mathbb{P}$-generic over $\mathbf{L}$. Then

$$
\mathbb{W}_{k}=\left\{\left\langle\xi, x_{\xi k}[G]\right\rangle: \xi<\omega_{1}\right\} \subseteq \omega_{1} \times 2^{\omega}
$$

is a set of definability class $\Pi_{k+2}^{\mathrm{HC}}$ in $\mathbf{L}[G]$ and in any transitive model $M \models \mathbf{Z F C}$ satisfying $\mathbf{L} \subseteq M \subseteq \mathbf{L}[G]$ and $\left\{x_{\xi k}[G]: \xi<\omega_{1}\right\} \subseteq M$.

Proof. By the theorem, it is true in $\mathbf{L}[G]$ that $\langle\xi, x\rangle \in \mathbb{W}_{k}$ iff

$$
\forall \alpha<\omega_{1} \exists T \in \mathbb{\square}_{\alpha}(\xi, k)(\alpha(\xi, k) \leq \alpha \Longrightarrow x \in[T]),
$$

which can be re-written as

$\forall \alpha<\omega_{1} \forall \mu<\omega_{1} \forall X \exists T \in X\left(\mu=\alpha(\xi, k) \wedge X=\mathbb{\square}_{\alpha}(\xi, k) \wedge \mu \leq \alpha \Longrightarrow x \in[T]\right)$.

Here the equality $\mu=\alpha(\xi, k)$ (with a fixed $k$ ) is $\Delta_{k+2}^{\mathrm{HC}}$ by Corollary 24.3, and so is the equality $X=\rrbracket_{\alpha}(\xi, k)$ by Corollary 24.3. It follows that the whole relation is $\Pi_{k+2}^{\mathrm{HC}}$, since the quantifier $\exists T \in X$ is bounded.

The next corollary is the first cornerstone in the proof of Theorem 1.1.

Corollary 32.3 (= (I), (II) of Theorem 1.1). Assume that $m<\omega$ and a set $G \subseteq \mathbb{P}$ is $\mathbb{P}$-generic over $\mathbf{L}$. Then $\omega^{\omega} \cap \mathbf{L}\left[G \|_{<m}\right]$ is a $\Sigma_{m+3}^{1}$ set in $\mathbf{L}[G]$, and it holds in $\mathbf{L}\left[G \|_{<m}\right]$ that there is a $\Delta_{m+3}^{1}$ wellordering of $\omega^{\omega}$ of length $\omega_{1}$.

Proof. If $\gamma<\omega_{1}$ then let $X_{\gamma n}=\left\langle x_{\xi k}[G]\right\rangle_{\xi<\gamma \wedge k<n}$; thus $X=X_{\gamma n}$ is a $\Pi_{n+1}^{\mathrm{HC}}$ relation in $\mathbf{L}[G]$ (with $\gamma, n, X$ as arguments) by Corollary 32.2. It follows that

$$
\omega^{\omega} \cap \mathbf{L}\left[G \pi_{<m}\right]=\left\{x \in \omega^{\omega}: \exists \gamma<\omega_{1}\left(x \in \mathbf{L}\left[X_{\gamma n}\right]\right)\right\}
$$

is a set in $\Sigma_{n+2}^{\mathrm{HC}}$, hence, a $\Sigma_{m+3}^{1}$ set in $\mathbf{L}[G]$. To define a required wellordering, if $x \in \omega^{\omega} \cap \mathbf{L}\left[G \Uparrow_{<m}\right]$ then let $\gamma(x)$ be the least $\gamma<\omega_{1}$ such that $x \in \mathbf{L}\left[X_{\gamma n}\right]$, and let $\nu(x)<\omega_{1}$ be the index of $x$ in the canonical wellordering of $\omega^{\omega}$ in $\mathbf{L}\left[X_{\gamma n}\right]$. Now we wellorder $\omega^{\omega} \cap \mathbf{L}\left[G \|_{<m}\right]$ according to the lexicographical ordering of triples $\langle\max \{\gamma(x), \nu(x)\}, \gamma(x), \nu(x)\rangle$. 


\section{Elementary equivalence}

Here we prove the following elementary equivalence theorem for key generic extensions. Compare to (III) of Theorem 1.1.

Theorem 33.1. Assume that $m<\omega$ and a set $G \subseteq \mathbb{P}$ is $\mathbb{P}$-generic over $\mathbf{L}$. Then $\mathbf{L}\left[G \pi_{<m}\right]$ is an elementary submodel of $\mathbf{L}[G]$ w.r.t. all $\Sigma_{m+2}^{1}$ formulas.

Proof. Suppose that this is not the case. Then there is a $\Pi_{m+1}^{1}$ formula $\varphi(r, x)$ with $r \in \omega^{\omega} \cap \mathbf{L}\left[G \|_{<m}\right]$ as the only parameter, and a real $x_{0} \in \omega^{\omega} \cap \mathbf{L}[G]$ such that $\varphi\left(r, x_{0}\right)$ is true in $\mathbf{L}[G]$ but there is no $x \in \omega^{\omega} \cap \mathbf{L}\left[G \|_{<m}\right]$ such that $\varphi(r, x)$ is true in $\mathbf{L}[G]$. By a version of Proposition 18.1(ii), we have $r=\mathbf{c}_{0}[G]$, where $\mathbf{c}_{0}$ is a small true $\left(\mathbb{P} \|_{<m}\right)$-real name. (See Section 31 on notation.) And there is a small true $\mathbb{P}$-real name $\mathbf{c}$ such that $x_{0}=\mathbf{c}[\underline{G}]$.

By Lemma 30.3, there is a multitree $\boldsymbol{p}_{0} \in G$ such that

(1) $\boldsymbol{p}_{0} \mathbb{P}$-forces $\varphi\left(\mathbf{c}_{0}[\underline{G}], \mathbf{c}[\underline{G}]\right) \wedge \neg \exists x \in \mathbf{L}\left[\underline{G} \|_{<m}\right] \varphi\left(\mathbf{c}_{0}[\underline{G}], x\right)$ over $\mathbf{L}$;

(2) $\boldsymbol{p}_{0}$ forc $\varphi\left(\mathbf{c}_{0}, \mathbf{c}\right)$, that is, $\boldsymbol{p}_{0}$ forc $\overrightarrow{\mathbb{\pi}} \mid \gamma_{0} \varphi\left(\mathbf{c}_{0}, \mathbf{c}\right)$, where $\gamma_{0}<\omega_{1}$ - and we can assume that $\boldsymbol{p}_{0} \in \mathbf{M T}\left(\overrightarrow{\mathbb{\top}}\left\lceil\gamma_{0}\right)\right.$ as well.

As $\mathbf{c}, \mathbf{c}_{0}$ are small names, there is an ordinal $\delta<\omega_{1}$ satisfying

(3) $\left|\mathbf{c}_{0}\right| \subseteq \delta \times m,|\mathbf{c}| \subseteq \delta \times \omega$, and $\left|\boldsymbol{p}_{0}\right| \subseteq \delta \times \omega$,

and we can enlarge $\gamma_{0}$, if necessary, using the equality $|\vec{\pi}|=\omega_{1} \times \omega$ of Lemma 24.2, to make sure that

(4) $\delta \times \omega \subseteq|\overrightarrow{\mathbb{\Pi}}| \gamma_{0} \mid$, that is, if $\eta<\delta$ and $k<\omega$ then $\langle\eta, k\rangle \in\left|\square_{\alpha^{\prime}}\right|$ for some $\alpha^{\prime}=\alpha^{\prime}(\eta, k)<\gamma_{0}$.

We are starting from here towards a contradiction.

Let $U$ consist of all multisequences of the form $\vec{\pi} \|_{\geq m}$, where

(A) $\overrightarrow{\boldsymbol{\pi}} \in \overrightarrow{\mathbf{s M F}}\left[\vec{\pi} \|_{<m}\right], \vec{\pi}\left\lceil\gamma_{0} \subset \overrightarrow{\boldsymbol{\pi}}\right.$, and hence $\boldsymbol{p}_{0} \in \mathbf{M T}(\overrightarrow{\boldsymbol{\pi}})$ by $(2)$;

and there is an ordinal $\zeta<\omega_{1}$ and a transformation $\boldsymbol{h} \in \mathrm{PERM}_{m-1}$ such that

(B) $\boldsymbol{h}=\boldsymbol{h}^{-1},|\boldsymbol{h}|=D \cup R$, and $\boldsymbol{h}$ maps $D$ onto $R$ and $R$ onto $D$, where $D=\delta \times[m, \omega), R=\left\{\langle\xi, m-1\rangle: \nu_{0} \leq \xi<\nu_{1}\right\}$, and $\delta<\nu_{0}<\nu_{1}<\omega_{1} ;$

(C) $\gamma_{0} \leq \zeta<\operatorname{dom} \overrightarrow{\boldsymbol{\pi}}$ and $\left.(\boldsymbol{h} \overrightarrow{\boldsymbol{\pi}})\right|_{\geq \zeta}=\overrightarrow{\boldsymbol{\pi}} \uparrow_{\geq \zeta}$, or equivalently $\boldsymbol{h}(\overrightarrow{\boldsymbol{\pi}}(\alpha))=\overrightarrow{\boldsymbol{\pi}}(\alpha)$ whenever $\zeta \leq \alpha<\operatorname{dom} \overrightarrow{\boldsymbol{\pi}}$. 
It follows from Lemma 25.2 that $U$ is a $\boldsymbol{\Sigma}_{m+1}^{\mathrm{HC}}$ set (with $\vec{\mathbb{}}\left\lceil\gamma_{0}, \delta\right.$ as parameters). Therefore by 23.4(ii) there is an ordinal $\gamma<\omega_{1}$ such that $\vec{\mathbb{}} \uparrow \gamma m$-decides $U$.

Case 1: $\left(\overrightarrow{\mathbb{\pi}}\lceil\gamma) \|_{>m} \in U\right.$. Basically this means that there is a transformation $\boldsymbol{h} \in \mathrm{PERM}_{m-1}$ such that (A), (B), (C) hold for $\boldsymbol{h}$ and $\overrightarrow{\boldsymbol{\pi}}=\vec{\mathbb{}} \uparrow \gamma$, via ordinals $\delta<\nu_{0}<\nu_{1}$ and $\gamma_{0}<\zeta<\gamma$ as in (B), (C).

Now, by Lemma 26.3 and (2), we have $\boldsymbol{p}_{0} \operatorname{forc}_{\vec{\pi} \uparrow \gamma} \varphi\left(\mathbf{c}_{0}, \mathbf{c}\right)$. We further get $\boldsymbol{h} \boldsymbol{p}_{0}$ forc $_{\boldsymbol{h} \vec{\pi}\lceil\gamma} \varphi\left(\boldsymbol{h} \mathbf{c}_{0}, \boldsymbol{h c}\right)$ by Theorem 29.1 because $\varphi$ is a $\mathscr{L} \Pi_{n+1}^{1}$ formula and $\boldsymbol{h}$ belongs to $\mathrm{PERM}_{m-1}$. However $\boldsymbol{h} \mathbf{c}_{0}=\mathbf{c}_{0}$ since $\left|\mathbf{c}_{0}\right| \cap|\boldsymbol{h}|=\varnothing$ by (B). Thus $\boldsymbol{p}_{0}^{\prime}$ forc $_{\vec{\pi}\lceil\gamma} \varphi\left(\mathbf{c}_{0}, \mathbf{c}^{\prime}\right)$ holds by Theorem 28.1 and $(\mathrm{C})$, where $\mathbf{c}^{\prime}=\boldsymbol{h} \mathbf{c}, \boldsymbol{p}_{0}^{\prime}=\boldsymbol{h} \boldsymbol{p}_{0}$.

Note that the common part $\left|\boldsymbol{p}_{0}\right| \cap\left|\boldsymbol{p}_{0}^{\prime}\right|$ of the domains of $\boldsymbol{p}_{0}, \boldsymbol{p}_{0}^{\prime}$ does not intersect $|\boldsymbol{h}|$ by (B) since $\left|\boldsymbol{p}_{0}\right| \subseteq \delta \times \omega$ by (3). It follows that $\boldsymbol{p}_{0}, \boldsymbol{p}_{0}^{\prime}$ are compatible, basically $\boldsymbol{p}=\boldsymbol{p}_{0} \cup \boldsymbol{p}_{0}^{\prime}$ is a multitree in $\mathbf{M T}(\overrightarrow{\mathbb{T}} \uparrow \gamma)$. Thus $\boldsymbol{p} \leqslant \boldsymbol{p}_{0}^{\prime}$ and still $\boldsymbol{p}$ forc $_{\vec{\pi}\lceil\gamma} \varphi\left(\mathbf{c}_{0}, \mathbf{c}^{\prime}\right)$. It follows by Lemma 30.3 that

(5) $\boldsymbol{p} \mathbb{P}$-forces $\varphi\left(\mathbf{c}_{0}[\underline{G}], \mathbf{c}^{\prime}[\underline{G}]\right)$ over $\mathbf{L}$.

However $\left|\mathbf{c}^{\prime}\right| \subseteq \omega_{1} \times m$ by construction because $|\mathbf{c}| \subseteq \delta \times \omega$ by (3), and hence $\mathbf{c}^{\prime}[\underline{G}] \in \mathbf{L}\left[\underline{G} \|_{<m}\right]$ is forced. Thus $\boldsymbol{p} \mathbb{P}$-forces $\exists x \in \mathbf{L}\left[\underline{G} \|_{<m}\right] \varphi\left(\mathbf{c}_{0}[\underline{G}], x\right)$ over $\mathbf{L}$ by (5), contrary to (1). The contradiction ends Case 1 .

Case 2: negative decision, no multisequence in $U$ extends $\left(\vec{\square}\lceil\gamma) \|_{\geq m}\right.$. We can assume that $\gamma>\gamma_{0}$. (Otherwise replace $\gamma$ by $\gamma_{0}+1$.) Let $\nu_{0}$ be the lest ordinal, bigger than $\delta$ and satisfying $|\vec{\pi}| \gamma \mid \subseteq \nu_{0} \times \omega$. Let $\nu_{1}=\nu_{0}+\omega$. Then countable sets $D=\delta \times[m, \infty)$ and $R$ as in (B) are defined and $D \cap R=\varnothing$, so we can fix a transformation $\boldsymbol{h} \in \mathrm{PERM}_{m-1}$ satisfying (B). Note that $D \subseteq$ $\delta \times \omega \subseteq|\overrightarrow{\mathbb{\pi}}| \gamma \mid$ by (4) but $R \cap|\overrightarrow{\mathbb{\pi}}| \gamma \mid=\varnothing$ by the choice of $\nu_{0}$.

Pick $\lambda<\omega_{1}$ such that $\lambda>\gamma>\gamma_{0}$. Then the multisequence $\vec{\phi}=\vec{\mathbb{}} \uparrow \lambda$ clearly satisfies (A), (B) and extends $\vec{\pi} \mid \gamma$. Our plan is now to slightly modify $\overrightarrow{\boldsymbol{Q}}$ in order to fulfill (C) as well, with $\zeta=\gamma$. Such a minor modification consists in the replacement of the $R$-part of $\vec{\phi}$ above $\gamma$ by the $\boldsymbol{h}$-copy of its $D$-part.

To present this in detail, recall that $\overrightarrow{\boldsymbol{\varphi}}=\vec{\mathbb{}} \mid \lambda=\left\langle\square_{\alpha}\right\rangle_{\alpha<\lambda}$, where each $\square_{\alpha}$ is a small multiforcing, whose domain $d_{\alpha}=\left|\square_{\alpha}\right| \subseteq \omega_{1} \times \omega$ is countable. If $\alpha<\gamma$ then put $\boldsymbol{\pi}_{\alpha}=\llbracket_{\alpha}$. Suppose that $\gamma \leq \alpha<\lambda$. Then $D \subseteq\left|\rrbracket_{\alpha}\right|$ by (4). Define a modified multiforcing $\boldsymbol{\pi}_{\alpha}$ such that

(a) $\left|\boldsymbol{\pi}_{\alpha}\right|=d_{\alpha} \cup R$ - note that $D \subseteq d_{\alpha} \subseteq\left|\boldsymbol{\pi}_{\alpha}\right|$ in this case because $D \subseteq|\overrightarrow{\mathbb{\pi}}| \gamma \mid$ by (4) (as $\left.\gamma_{0} \leq \gamma\right)$, and hence $D \subseteq d_{\alpha}=\left|\mathbb{\square}_{\alpha}\right|$ (as $\alpha \geq \gamma$ ),

(b) if $\langle\xi, k\rangle \in d_{\alpha} \backslash R$ then $\pi_{\alpha}(\xi, k)=\rrbracket_{\alpha}(\xi, k)$,

(c) if $\langle\xi, k\rangle \in D$, so $\boldsymbol{h}(\xi, k)=\langle\eta, m-1\rangle \in R$, then $\boldsymbol{\pi}_{\alpha}(\eta, m-1)=\mathbb{\square}_{\alpha}(\xi, k)$.

We claim that $\overrightarrow{\boldsymbol{\pi}}=\left\langle\boldsymbol{\pi}_{\alpha}\right\rangle_{\alpha<\lambda}$ is a multisequence, that is, if $\alpha<\beta<\lambda$ then $\boldsymbol{\pi}_{\alpha} \sqsubset$ $\boldsymbol{\pi}_{\beta}$. This amounts to the folowing: if $\langle\eta, k\rangle \in\left|\boldsymbol{\pi}_{\alpha}\right|$ then $\boldsymbol{\pi}_{\alpha}(\eta, k) \sqsubset \boldsymbol{\pi}_{\beta}(\eta, k)$. Note that $\boldsymbol{\pi}_{\alpha}(\eta, k)=\rrbracket_{\alpha}(\eta, k)$ in case $\langle\eta, k\rangle \notin R$. 
Thus it remains to check that $\boldsymbol{\pi}_{\alpha}(\eta, m-1) \sqsubset \boldsymbol{\pi}_{\beta}(\eta, m-1)$ whenever $\alpha<$ $\beta<\lambda,\langle\eta, m-1\rangle=\boldsymbol{h}(\xi, k) \in R \cap\left|\boldsymbol{\pi}_{\alpha}\right|$, and $\langle\xi, k\rangle \in D$. If now $\alpha<\gamma$ then $R \cap\left|\boldsymbol{\pi}_{\alpha}\right|=\varnothing$ by the choice of $\nu_{0}$, so it remains to consider the case when $\gamma \leq \alpha$. Then the pairs $\langle\xi, k\rangle,\langle\eta, m-1\rangle$ belong to $\left|\boldsymbol{\pi}_{\alpha}\right|$ by construction, and we have $\boldsymbol{\pi}_{\alpha}(\eta, m-1)=\mathbb{\square}_{\alpha}(\xi, k)$ and $\boldsymbol{\pi}_{\beta}(\eta, m-1)=\mathbb{\square}_{\beta}(\xi, k)$. Therefore $\boldsymbol{\pi}_{\alpha}(\xi, m) \sqsubset \boldsymbol{\pi}_{\beta}(\xi, m)$ since $\vec{\square}$ is a multisequence, and we are done.

Now we claim that the multisequence $\overrightarrow{\boldsymbol{\pi}}=\left\langle\boldsymbol{\pi}_{\alpha}\right\rangle_{\alpha<\lambda}$ satisfies (A), (B), (C). Indeed as the difference between each $\boldsymbol{\pi}_{\alpha}$ and the corresponding $\mathbb{\square}_{\alpha}$ is fully located in the domain $R=\left\{\langle\xi, m-1\rangle: \nu_{0} \leq \xi<\nu_{1}\right\}$, we have $\overrightarrow{\boldsymbol{\pi}} \|_{<m-1}=$ $\overrightarrow{\boldsymbol{\varphi}} \|_{<m-1}$, therefore $\overrightarrow{\boldsymbol{\pi}} \in \overrightarrow{\mathbf{s M F}}\left[\vec{\Pi} \|_{<m}\right]$. We also note that $\overrightarrow{\boldsymbol{\pi}}\lceil\gamma=\overrightarrow{\boldsymbol{\varphi}}\lceil\gamma$ by construction, hence $\overrightarrow{\mathbb{\pi}}\lceil\gamma=\overrightarrow{\boldsymbol{\varphi}}\lceil\gamma \subset \overrightarrow{\boldsymbol{\pi}}$. This implies (A).

We also have (B) by construction. We finally claim that (C) is satisfied with $\zeta=\gamma$, that is, if $\gamma \leq \alpha<\lambda$ then $\boldsymbol{h} \cdot \boldsymbol{\pi}_{\alpha}=\boldsymbol{\pi}_{\alpha}$. Indeed we have $D \cup R \subseteq\left|\boldsymbol{\pi}_{\alpha}\right|$, see (a). Now the invariance of $\boldsymbol{\pi}_{\alpha}$ under $\boldsymbol{h}$ holds by (b), (c).

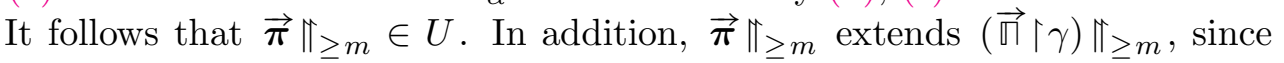
$\vec{\mathbb{}} \uparrow \gamma \subset \overrightarrow{\boldsymbol{\pi}}$. But this contradicts the Case 2 assumption.

To conclude, either case leads to a contradiction, proving the theorem.

\section{Non-wellorderability}

We finally prove that the reals are not wellorderable by a (lightface) analytically definable relation in $\mathbb{P}$-generic extensions, that is, (IV) of Theorem 1.1.

Theorem 34.1. Assume that $m<\omega$ and a set $G \subseteq \mathbb{P}$ is $\mathbb{P}$-generic over $\mathbf{L}$. Then it is true in $\mathbf{L}[G]$ that the reals are not wellorderable by an analytically definable relation.

Proof. Suppose to the contrary that, in $\mathbf{L}[G]$, a $\Sigma_{m+2}^{1}$ relation « strictly wellorders $\omega^{\omega}, m \geq 1$. Let $\psi(x, y)$ be a parameter-free $\Sigma_{m+2}^{1}$ formula, which defines $\ll$, so that $x \ll y$ iff $\psi(x, y)$ in $\mathbf{L}[G]$. Note that $\ll$ is essentially a $\Delta_{m+2}^{1}$ relation, since $x \ll y \Longleftrightarrow y \ll x \wedge x \neq y$.

Of all nonconstructible reals $x_{\xi m}[G], \xi<\omega_{1}$, there is a $\ll$-least one. We suppose that $x_{0 m}[G]$ is such. (If it is some $x_{\xi_{0} m}[G], \xi_{0} \neq 0$, then the arguments suitably change in obvious way.) That is, $x_{0 m}[G] \ll x_{\xi m}[G]$ whenever $\xi>0$. Accordingly there is a multitree $\boldsymbol{p}_{0} \in G$ that $\mathbb{P}$-forces, over $\mathbf{L}$, that

(1) $\ll$ (that is, the relation defined by $\psi$ ) is a wellordering of $\omega^{\omega}$, and

(2) $\forall \xi>0\left(x_{0 m}[\underline{G}] \ll x_{\xi m}[\underline{G}]\right)$.

Therefore, if $\xi>0$ then $\boldsymbol{p}_{0} \mathbb{P}$-forces $\dot{\boldsymbol{x}}_{0 m}[\underline{G}] \ll \dot{\boldsymbol{x}}_{\xi m}[\underline{G}]$ ) over $\mathbf{L}$. (We make use of the real names $\dot{\boldsymbol{x}}_{\xi k}$ introduced by 13.6, 13.7.)

By Lemma 30.3, we can assume that $\boldsymbol{p}_{0}$ forc $(1) \wedge(2)$, so that in fact we have $\boldsymbol{p}_{0} \operatorname{forc}_{\vec{\pi}\left\lceil\gamma_{0}\right.}(1) \wedge(2)$, for some $\gamma_{0}<\omega_{1}$. Then $\boldsymbol{p}_{0} \in \mathbf{M T}\left(\overrightarrow{\mathbb{\top}}\left\lceil\gamma_{0}\right)=\mathbf{M T}\left(\square_{<\gamma_{0}}\right)\right.$, 
where $\square_{<\gamma_{0}}=\bigcup_{\xi<\gamma_{0}}^{\mathrm{cw}} \rrbracket_{\xi}$ is a small multiforcing. Let $\delta<\omega_{1}$ be the least ordinal satisfying $\left|\square_{<\gamma_{0}}\right| \subseteq \delta \times \omega$. It follows then that $\left|\boldsymbol{p}_{0}\right| \subseteq \delta \times \omega$.

By Lemma 30.4, there is an ordinal $\gamma_{1}, \gamma_{0}<\gamma_{1}<\omega_{1}$, such that if $\xi<\omega_{1}$ then $\left.\boldsymbol{p}_{0}{\text { forc } \overrightarrow{\mathbb{\pi}}\left\lceil\gamma_{1}\right.}_{(\boldsymbol{x}} \ll \dot{\boldsymbol{x}}_{0 m}\right)^{-}$. We can enlarge $\gamma_{1}$, if necessary, using Lemma 24.2, to make sure that $\langle 0, m\rangle \in|\overrightarrow{\mathbb{\Pi}}| \gamma_{1} \mid$, that is, $\langle 0, m\rangle \in\left|\mathbb{\square}_{\alpha^{\prime}}\right|$ for some $\alpha^{\prime}<\gamma_{1}$.

If $\xi<\omega_{1}$ then let $\boldsymbol{h}_{\xi} \in \mathrm{PERM}_{m}$ be the permutation of $\langle 0, m\rangle$ and $\langle\xi, m\rangle$, such that $\left|\boldsymbol{h}_{\xi}\right|=\{\langle 0, m\rangle,\langle\xi, m\rangle\}, \boldsymbol{h}_{\xi}(0, m)=\langle\xi, m\rangle, \boldsymbol{h}_{\xi}(\xi, m)=\langle 0, m\rangle, \boldsymbol{h}_{\xi}(\eta, n)=$ $\langle\eta, n\rangle$ for any pair $\langle\eta, n\rangle$ different from both $\langle 0, m\rangle$ and $\langle\xi, m\rangle$.

The remainder of the proof is very similar to the proof of Theorem 33.1. Let $U$ consist of all multisequences of the form $\vec{\pi} \|_{\geq m}$, where

(A) $\overrightarrow{\boldsymbol{\pi}} \in \overrightarrow{\mathbf{s M F}}\left[\vec{\mathbb{}} \|_{<m}\right], \vec{\mathbb{}}\left\lceil\gamma_{1} \subset \overrightarrow{\boldsymbol{\pi}}\right.$, and hence $\boldsymbol{p}_{0} \in \mathbf{M T}(\overrightarrow{\boldsymbol{\pi}})$ by $(2) ;$

and there exist ordinals $\xi, \zeta<\omega_{1}$ such that

(B) $\delta<\xi<\operatorname{dom} \overrightarrow{\boldsymbol{\pi}}$;

(C) $\gamma_{1} \leq \zeta<\operatorname{dom} \overrightarrow{\boldsymbol{\pi}}$ and $\left(\boldsymbol{h}_{\xi} \overrightarrow{\boldsymbol{\pi}}\right) \uparrow_{\geq \zeta}=\overrightarrow{\boldsymbol{\pi}} \uparrow_{\geq \zeta}$, or equivalently $\boldsymbol{h}_{\xi} \cdot(\overrightarrow{\boldsymbol{\pi}}(\alpha))=\overrightarrow{\boldsymbol{\pi}}(\alpha)$ whenever $\zeta \leq \alpha<\operatorname{dom} \vec{\pi}$.

It follows from Lemma 25.2 that $U$ is a $\boldsymbol{\Sigma}_{m+1}^{\mathrm{HC}}$ set (with $\vec{\pi} \uparrow \gamma_{1}$ as a parameter). Therefore by 23.4(ii) there is an ordinal $\gamma<\omega_{1}$ such that $\overrightarrow{\mathbb{\top}} \uparrow \gamma m$-decides $U$.

Case 1: $(\overrightarrow{\mathbb{T}} \uparrow \gamma) \|_{\geq m} \in U$. Basically this means that $\gamma>\gamma_{1}$ and there are ordinals $\xi<\omega_{1}$ and $\zeta<\gamma$ such that (A), (B), (C) hold for $\xi$ and the multisequence

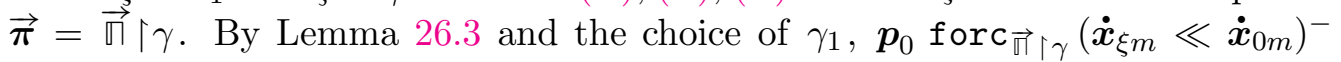
holds. This implies $\boldsymbol{h}_{\xi} \boldsymbol{p}_{0}$ forc $_{\boldsymbol{h}_{\xi} \vec{\nabla}\lceil\gamma}\left(\dot{\boldsymbol{x}}_{0 m} \ll \dot{\boldsymbol{x}}_{\xi m}\right)^{-}$by Theorem 29.1 because $\left(\dot{\boldsymbol{x}}_{0 m} \ll \dot{\boldsymbol{x}}_{\xi m}\right)^{-}$is a $\mathscr{L} \Pi_{m+2}^{1}$ formula and $\boldsymbol{h}_{\xi}$ belongs to $\mathrm{PERM}_{m}$. We conclude that $\boldsymbol{p}_{0}^{\prime} \operatorname{forc}_{\vec{\pi} \backslash \gamma}\left(\dot{\boldsymbol{x}}_{0 m} \ll \dot{\boldsymbol{x}}_{\xi m}\right)^{-}$by $(\mathrm{C})$ and Theorem 28.1, where $\boldsymbol{p}_{0}^{\prime}=\boldsymbol{h}_{\xi} \boldsymbol{p}_{0}$.

Note that $\langle\xi, m\rangle \notin\left|\boldsymbol{p}_{0}\right|$ by (B). It follows by the definition of $\boldsymbol{h}_{\xi}$ that $\boldsymbol{p}_{0}, \boldsymbol{p}_{0}^{\prime}$ are compatible, basically $\boldsymbol{p}=\boldsymbol{p}_{0} \cup \boldsymbol{p}_{0}^{\prime}$ is a multitree in $\mathbf{M T}\left(\overrightarrow{\mathbb{\Pi}}\lceil\gamma)\right.$ and $\boldsymbol{p} \leqslant \boldsymbol{p}_{0}$,

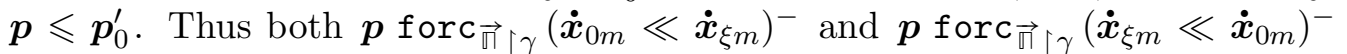
hold, contrary to Lemma 30.2(iv). The contradiction ends Case 1.

Case 2: negative decision, no multisequence in $U$ extends $(\overrightarrow{\mathbb{G}} \uparrow \gamma) \|_{\geq m}$. We can assume that $\gamma>\gamma_{1}$ (otherwise replace $\gamma$ by $\gamma_{1}+1$ ). Note that $\vec{\mathbb{}} \uparrow \gamma=$ $\left\langle\square_{\alpha}\right\rangle_{\alpha<\gamma}$, where each $\square_{\alpha}$ is a small multiforcing, whose domain $d_{\alpha}=\left|\rrbracket_{\alpha}\right| \subseteq \omega_{1} \times \omega$ is countable. Therefore the set $d=|\vec{\pi}| \gamma \mid=\bigcup_{\alpha<\gamma} d_{\alpha}$ is still countable. Pick an ordinal $\xi, \delta<\xi<\omega_{1}$, such that $\langle\xi, m\rangle \notin d$. Finally pick an ordinal $\lambda$, $\gamma<\lambda<\omega_{1}$. Then $\overrightarrow{\boldsymbol{\phi}}=\overrightarrow{\mathbb{\Pi}} \mid \lambda$ (as $\overrightarrow{\boldsymbol{\pi}}$ ) and $\xi$ clearly satisfy (A) and (B), and $\overrightarrow{\boldsymbol{\varphi}}$ extends $\overrightarrow{\mathbb{\Pi}} \mid \gamma$. Let's somewhat modify $\vec{\varphi}$ in order to fulfill (C) as well.

As above, $\vec{\varphi}=\vec{\mathbb{}} \uparrow \lambda=\left\langle\square_{\alpha}\right\rangle_{\alpha<\lambda}$, each $\rrbracket_{\alpha}$ is a small multiforcing, and its domain $d_{\alpha}=\left|\mathbb{\square}_{\alpha}\right| \subseteq \omega_{1} \times \omega$ is countable. If $\alpha<\gamma$ then put $\boldsymbol{\pi}_{\alpha}=\mathbb{\square}_{\alpha}$. Suppose that $\gamma \leq \alpha<\lambda$. Then $\alpha \geq \gamma_{1}$, and hence $\langle 0, m\rangle \in\left|\square_{\alpha}\right|$ by the choice of $\gamma_{1}$. 
Define a modified multiforcing $\boldsymbol{\pi}_{\alpha}$ such that $\left|\boldsymbol{\pi}_{\alpha}\right|=d_{\alpha} \cup\{\langle\xi, m\rangle\}$, if $\langle\eta, k\rangle \in$ $d_{\alpha} \backslash\{\langle\xi, m\rangle\}$ then $\boldsymbol{\pi}_{\alpha}(\eta, k)=\mathbb{\square}_{\alpha}(\eta, k)$, and finally $\boldsymbol{\pi}_{\alpha}(\xi, m)=\mathbb{\square}_{\alpha}(0, m)$.

We claim that $\overrightarrow{\boldsymbol{\pi}}=\left\langle\boldsymbol{\pi}_{\alpha}\right\rangle_{\alpha<\lambda}$ is a multisequence, that is, if $\alpha<\beta<\lambda$ then $\boldsymbol{\pi}_{\alpha} \sqsubset \boldsymbol{\pi}_{\beta}$. This amounts to the folowing: if $\langle\eta, k\rangle \in\left|\boldsymbol{\pi}_{\alpha}\right|$ then $\boldsymbol{\pi}_{\alpha}(\eta, k) \sqsubset$ $\boldsymbol{\pi}_{\beta}(\eta, k)$. Note that $\boldsymbol{\pi}_{\alpha}(\eta, k)=\mathbb{T}_{\alpha}(\eta, k)$ whenever $\langle\eta, k\rangle \neq\langle\xi, m\rangle$. Thus it remains to check that $\boldsymbol{\pi}_{\alpha}(\xi, m) \sqsubset \boldsymbol{\pi}_{\beta}(\xi, m)$ given $\alpha<\beta<\lambda$ such that $\langle\xi, m\rangle \in$ $\left|\boldsymbol{\pi}_{\alpha}\right|$. If $\alpha<\gamma$ then $\left|\boldsymbol{\pi}_{\alpha}\right|=\left|\rrbracket_{\alpha}\right|=d_{\alpha}$ by construction, and hence $\langle\xi, m\rangle \notin d_{\alpha}$ by the choice of $\xi$. It remains to consider the case $\gamma \leq \alpha<\lambda$. Then $\langle 0, m\rangle \in d_{\alpha}$ (see above), hence the pairs $\langle 0, m\rangle,\langle\xi, m\rangle$ belong to $\left|\boldsymbol{\pi}_{\alpha}\right|$ by construction, and then obviously belong to $\left|\boldsymbol{\pi}_{\beta}\right|$ as $\alpha<\beta$. Now $\boldsymbol{\pi}_{\alpha}(\xi, m)=\mathbb{\square}_{\alpha}(0, m)$ and $\boldsymbol{\pi}_{\beta}(\xi, m)=$ $\square_{\beta}(0, m)$, and we have $\boldsymbol{\pi}_{\alpha}(\xi, m) \sqsubset \boldsymbol{\pi}_{\beta}(\xi, m)$ since $\vec{\pi}$ is a multisequence.

Now we claim that the multisequence $\overrightarrow{\boldsymbol{\pi}}=\left\langle\boldsymbol{\pi}_{\alpha}\right\rangle_{\alpha<\lambda}$ satisfies (A), (B), (C) with $\zeta=\gamma$. If $\alpha<\lambda$ then the difference between $\mathbb{\square}_{\alpha}$ and $\pi_{\alpha}$ is located in the one-element domain $\{\langle\xi, m\rangle\}$, therefore $\boldsymbol{\pi}_{\alpha} \mathbb{\pi}_{<m}=\mathbb{\square}_{\alpha} \|_{<m}$. It follows that $\overrightarrow{\boldsymbol{\pi}} \|_{<m}=\left(\overrightarrow{\mathbb{\Pi}}\lceil\lambda) \|_{<m}\right.$, hence $\overrightarrow{\boldsymbol{\pi}} \in \overrightarrow{\mathbf{s M F}}\left[\overrightarrow{\mathbb{\pi}} \|_{<m}\right]$. We further have $\overrightarrow{\boldsymbol{\pi}}\lceil\gamma=\overrightarrow{\mathbb{\Pi}}\lceil\gamma$ by construction. Thus $\overrightarrow{\mathbb{\pi}}\left\lceil\gamma \subset \overrightarrow{\boldsymbol{\pi}}\right.$, hence $\vec{\mathbb{}}\left\lceil\gamma_{1} \subset \overrightarrow{\boldsymbol{\pi}}\right.$, and we have (A).

We also have (B) and (C) (with $\zeta=\gamma$ ) by construction.

Thus $\overrightarrow{\boldsymbol{\pi}} \|_{\geq m} \in U$. In addition, $\overrightarrow{\boldsymbol{\pi}} \|_{\geq m}$ extends $(\overrightarrow{\mathbb{\pi}} \uparrow \gamma) \|_{\geq m}$, since even more $\overrightarrow{\mathbb{\Pi}} \mid \gamma \subset \vec{\pi}$ by construction. But this contradicts to the Case 2 assumption.

To conclude, either case leads to a contradiction, proving the theorem.

\section{Proof of the main theorem}

Proof (Theorem 1.1). We consider a $\mathbb{P}$-generic extension $\mathbf{L}[G]$ of $\mathbf{L}$ and present it in the form $\mathbf{L}[G]=\mathbf{L}[X]$ as in Section 31, where $X=\left\langle x_{\xi k}\right\rangle_{\langle\xi, k\rangle \in \omega_{1} \times \omega}$, and each $x_{\xi k}=x_{\xi k}[G]$ is a real in $2^{\omega} \cap \mathbf{L}[G]$. We also consider the subextensions $\mathbf{L}\left[G \Uparrow_{<m}\right]=\mathbf{L}\left[\left\langle x_{\xi k}\right\rangle_{\xi<\omega_{1} \wedge k<m}\right]$ of $\mathbf{L}[G]=\mathbf{L}[X]$. Then (I) and (II) of Theorem 1.1 hold by Corollary 32.3, (III) holds by Theorem 33.1, and finally (IV) holds by Theorem 34.1. 


\section{References}

[1] Uri Abraham. A minimal model for $\neg C H$ : iteration of Jensen's reals. Trans. Am. Math. Soc., 281:657-674, 1984.

[2] Uri Abraham. Minimal model of " $\aleph_{1}^{L}$ is countable" and definable reals. Adv. Math., 55:75-89, 1985.

[3] Joan Bagaria and Vladimir Kanovei. On coding uncountable sets by reals. Math. Log. Q., 56(4):409-424, 2010.

[4] Ali Enayat. On the Leibniz-Mycielski axiom in set theory. Fundam. Math., 181(3):215-231, 2004.

[5] J. Hadamard, R. Baire, H. Lebesgue, and E. Borel. Cinq lettres sur la théorie des ensembles. Bull. Soc. Math. Fr., 33:261-273, 1905.

[6] Leo Harrington. The constructible reals can be anything. Preprint dated May 1974 with several addenda dated up to October 1975:

(A) Models where Separation principles fail, May 74;

(B) Separation without Reduction, April 75;

(C) The constructible reals can be (almost) anything, Part II, May 75.

[7] Kai Hauser and Ralf-Dieter Schindler. Projective uniformization revisited. Ann. Pure Appl. Logic, 103(1-3):109-153, 2000.

[8] Thomas Jech. Set theory. Springer-Verlag, Berlin-Heidelberg-New York, The third millennium revised and expanded edition, 2003.

[9] R.B. Jensen and R.M. Solovay. Some applications of almost disjoint sets. In Yehoshua Bar-Hillel, editor, Math. Logic Found. Set Theory, Proc. Int. Colloqu., Jerusalem 1968, pages 84-104. North-Holland, Amsterdam-London, 1970.

[10] Ronald Jensen. Definable sets of minimal degree. In Yehoshua Bar-Hillel, editor, Math. Logic Found. Set Theory, Proc. Int. Colloqu., Jerusalem 1968, pages 122-128. North-Holland, Amsterdam-London, 1970.

[11] Ronald B. Jensen and Havard Johnsbraten. A new construction of a nonconstructible $\Delta_{3}^{1}$ subset of $\omega$. Fundam. Math., 81:279-290, 1974.

[12] V. Kanovei and V. Lyubetsky. A countable definable set of reals containing no definable elements. ArXiv e-prints, August 2014.

[13] V. Kanovei and V. Lyubetsky. A definable $\mathrm{E}_{0}$-class containing no definable elements. Archive of Mathematical Logic, 54(5):711-723, 2015.

[14] V.G. Kanovei. On the independence of some propositions of descriptive set theory and second-order arithmetic. Sov. Math., Dokl., 16:937-940, 1975.

[15] V.G. Kanovei and V.A. Lyubetsky. An effective minimal encoding of uncountable sets. Sib. Math. J., 52(5):854-863, 2011. 
[16] Vladimir Kanovei. On the nonemptiness of classes in axiomatic set theory. Math. USSR, Izv., 12:507-535, 1978.

[17] Vladimir Kanovei. The set of all alalytically definable sets of natural numbers can be defined analytically. Math. USSR Izvestija, 15(3):469-500, 1980.

[18] Vladimir Kanovei. A version of the Jensen-Johnsbråten coding at arbitrary level $n \geq 3$. Arch. Math. Logic, 40(8):615-628, 2001.

[19] Vladimir Kanovei and Vassily Lyubetsky. A definable $E_{0}$ class containing no definable elements. Archive of Mathematical Logic, 54(5):711-723, 2015.

[20] Vladimir Kanovei and Vassily Lyubetsky. Counterexamples to countable-section $\Pi_{2}^{1}$ uniformization and $\Pi_{3}^{1}$ separation. Ann. Pure Appl. Logic, 167(3):262-283, 2016.

[21] Alexander S. Kechris. Classical descriptive set theory. Springer-Verlag, New York, 1995.

[22] N. Lusin. Sur le problème de M. J. Hadamard d'uniformisation des ensembles. C. R. Acad. Sci., Paris, 190:349-351, 1930.

[23] Nicolas Lusin. Sur le problème de M. Jacques Hadamard d'uniformisation des ensembles. ${ }^{6}$ Mathematica, Cluj, 4:54-66, 1930.

[24] Yiannis N. Moschovakis. Descriptive set theory. Studies in Logic and the Foundations of Mathematics, Vol. 100. Amsterdam, New York, Oxford: North-Holland Publishing Company, 637 p., 1980.

\footnotetext{
${ }^{6}$ Luzin grants the uniformization problem to Hadamard with a reference to Hadamard's observations related to the axiom of choice in the famous Cinq Lettres [5].
} 


\section{Index}

a.d. (trees), 6

antichain, 7

avoids, 24

base, base $(\mathbb{P}), 7$

canonical homeomorphism

$$
\begin{aligned}
& \boldsymbol{h}_{S T}, 7 \\
& \boldsymbol{h}_{T}, 7
\end{aligned}
$$

Cohen forcing, $\mathbb{P}_{\text {coh }}, 7$

componentwise union

$$
\boldsymbol{\pi} \cup^{\mathrm{cw}} \boldsymbol{Q}, 10
$$

$\bigcup^{\mathrm{cw}} \overrightarrow{\boldsymbol{\pi}}=\bigcup_{\alpha<\lambda}^{\mathrm{cw}} \boldsymbol{\pi}_{\alpha}, 10$

CTM, countable transitive model, 39

decision

$m$-decides, 31

negative, 31

positive, 31

dense, 8,10

diamond sequences

$$
\begin{aligned}
& \overrightarrow{\boldsymbol{\pi}}\lceil\mu, m\rceil,\lceil\mu, m\rceil^{+}, 34 \\
& m\lceil\mu\rceil, p\lceil\mu\rceil, \delta\lceil\mu\rceil, 33 \\
& \overrightarrow{\boldsymbol{\pi}}\lceil\mu\rceil, D\lceil\mu\rceil, z\lceil\mu\rceil, 32
\end{aligned}
$$

directly forces, 22

domain-continuous, 29

finite splitting system, FSS, 8

empty system, $\boldsymbol{\Lambda}, 8$

extension proper, $\psi \prec \varphi, 8$

extension, $\psi \preccurlyeq \varphi, 8$

height, hgt $(\varphi), 8$

over $\mathbb{P}, \mathbf{F S S}(\mathbb{P}), 8$

tree occurs in, 8

forcing

forc, 39

wforc, 39

formula

$\mathscr{L}$-formula, 38

$$
\begin{aligned}
& \mathscr{L} \Sigma_{n}^{1}, \mathscr{L} \Pi_{n}^{1}, \mathscr{L}(\Sigma+\Pi)_{n}^{1}, 38 \\
& \varphi^{-}, 38
\end{aligned}
$$

generic

$\mathbb{P}$-generic real, 8

key elements

$\mathbb{C}, 34$

$\mathbb{C}^{\prime}, 37$

key maps $\mu_{m}, 34$

$\mu_{m}(\gamma), 34$

$\mathbb{P}, 36$

$\mathbb{P}_{<\gamma}, 36$

$\mathbb{P}_{\geq \gamma}, 36$

ㅁ, 36

$\overrightarrow{\mathbb{\pi}}, 34$

$\mathbb{\square}_{\alpha}, 34$

$\mathbb{\square}_{<\gamma}, 36$

$\mathbb{\square}_{\geq \gamma}, 36$

layer restriction
$\overrightarrow{\mathbf{s M F}} \|_{<m}, 31$
$\overrightarrow{\mathbf{s M F}} \|_{\geq m}, 31$
$\overrightarrow{\mathbf{s M F}} \|_{m}, 31$
$\overrightarrow{\mathbf{s M F}}_{\omega_{1}} \|_{<m}, 31$
$\overrightarrow{\mathbf{s M F}}_{\omega_{1}} \|_{\geq m}, 31$
$\overrightarrow{\mathbf{s M F}}_{\omega_{1}} \|_{m}, 31$
sMF $\|_{<m}, 31$
$\mathbf{s M F} \|_{\geq m}, 31$
$\mathbf{s M F} \|_{m}, 31$
$\mathbf{M F} \pi_{m}, 31$
$\mathbf{M F} \pi_{<m}, 31$
$\mathbf{M F} \pi_{\geq m}, 31$
$\mathbf{M T} \pi_{<m}, 31$
$\mathbf{M T} \|_{\geq m}, 31$
$\mathbf{M T} \|_{m}, 31$
$\boldsymbol{M T}(\boldsymbol{\pi}) \|_{<m}, 31$
$\mathbf{M T}(\boldsymbol{\pi}) \Uparrow_{m}, 31$ 


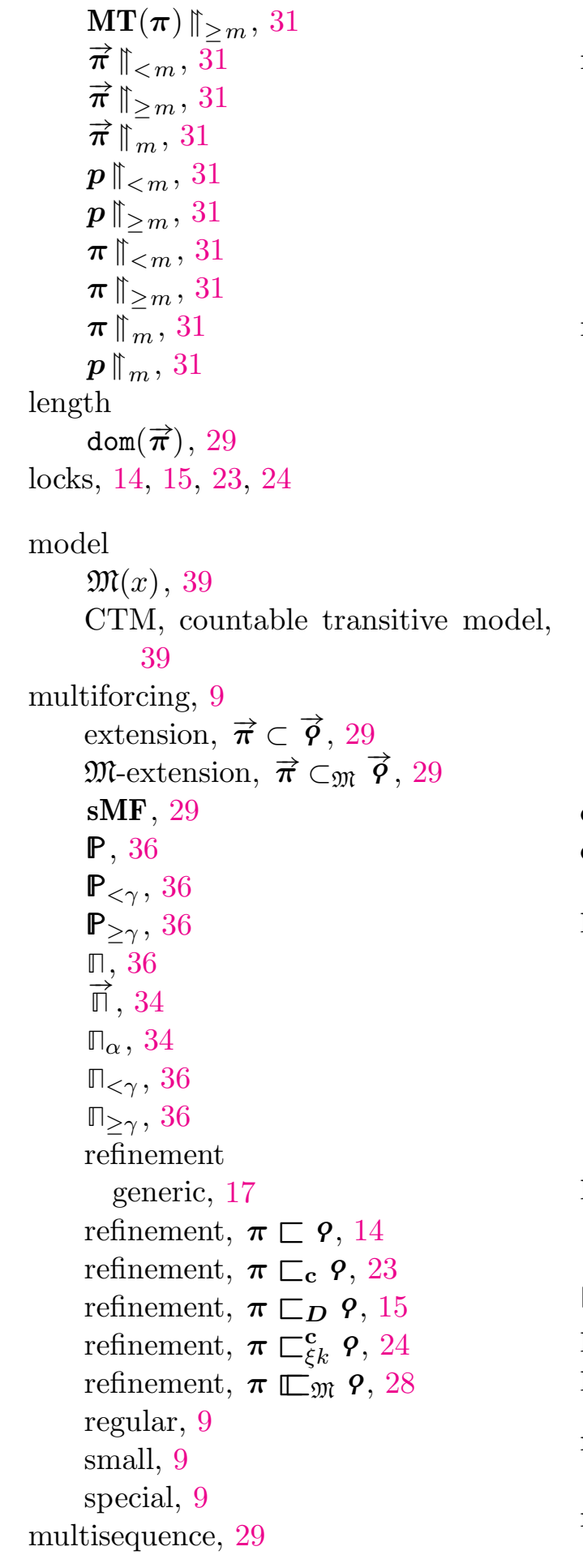

key multisequence, 35

multisystem, 11

2wise disjoint, 11

extension, $\boldsymbol{\psi} \preccurlyeq \boldsymbol{\varphi}, 11$

$h^{\varphi}(\xi, k, m), 11$

$\mathbf{M S}(\boldsymbol{\pi}), 11$

$\boldsymbol{\pi}$-multisystem, 11

$T_{\xi k, m}^{\varphi}(s), 11$

multitree, 9

MT, 9

$\mathbf{M T}(\boldsymbol{\pi}), 10$

$\operatorname{MT}(\vec{\pi}), 29$

disjoint union, $\boldsymbol{p} \cup \boldsymbol{q}, 19$

empty multitree, $\boldsymbol{\Lambda}, 9$

$\boldsymbol{\pi}$-multitree, 10

restriction, $\boldsymbol{p}\lceil X, 20$

somewhere almost disjoint, s.a.d., 9

$[\boldsymbol{p}], 9$

$T_{\xi k}^{p}, 9$

occurs, 8

open, 8, 10

perfect-tree forcing, PTF, 7

base, base $(\mathbb{P}), 7$

refinement, $\mathbb{P} \sqsubset \mathbb{Q}, 13$

refinement, $\mathbb{P} \sqsubset_{D} \mathbb{Q}, 14$

regular, 7

small, 7

special, 7

permutation

PERM, 44

action, 44

P-generic, 8

pre-dense, 8, 10

principal generic reals, $x_{\xi k}[G], 10$

real

P-generic, 8

real name, 21 
evaluation, $\mathbf{c}[G], 21$

$\overrightarrow{\boldsymbol{\pi}}$-real name, 29

true, 29

$\boldsymbol{\pi}$-real name, 21

true, 21

small, 21

true $\boldsymbol{\pi}$-real name, 21

reals

$\dot{\boldsymbol{x}}_{\xi k}, 22$

principal generic reals, $x_{\xi k}[G], 10$ refinement

generic, 17

locks, 14

refinement, $\mathbb{P} \sqsubset{ }_{D} \mathbb{Q}, 14$

refinement, $\boldsymbol{\pi} \sqsubset_{\mathbf{c}} \boldsymbol{Q}, 23$

refinement, $\pi \sqsubset_{D} \boldsymbol{Q}, 15$

refinement, $\boldsymbol{\pi} \sqsubset_{\xi k}^{\mathbf{c}} \boldsymbol{Q}, 24$

refinement, $\boldsymbol{\pi} \square_{\mathfrak{M}} \boldsymbol{\varphi}, 28$

regular, 7, 9

s.a.d., somewhere a.d., 9

set

$\mathbb{C}, 34$

$\mathbb{C}^{\prime}, 37$

set of multitrees

dense, 10

open, 10

pre-dense, 10

set of trees

dense, 8

open, 8

pre-dense, 8

sets $\Upsilon_{m}(p), 33$

small, 7,9

s.a.d., somewhere a.d., 9

somewhere almost disjoint, s.a.d., 9

special, 7,9

splitting, 6

stem, $\operatorname{stem}(T), 6$

strings, 6

empty string, $\Lambda, 6$ $\gamma$-tail, 42

tree, 6

$\operatorname{tree}(X), 6$

almost disjoint, 6

a.d. trees, 6

$\boldsymbol{Q}_{\xi k, m}^{\circledR \triangleright}, 17$

$\boldsymbol{Q}_{\xi k, m}^{\Phi \triangleright}(s), 17$

$T_{\xi k}^{p}, 9$

universal formula, $\mathbf{u n}_{m}(p, x), 33$

$2^{n}, 6$

$\leqslant \mathbf{L}, 32$

$\operatorname{base}(\mathbb{P}), 7$

$\mathbb{C}, 34$

$\mathbb{C}^{\prime}, 37$

$\mathbf{c}[G], 21$

$|\mathbf{c}|, 21$

$D\lceil\mu\rceil, 32$

$D_{\boldsymbol{q}}^{|\boldsymbol{u}|}, 15$

forc , 39

FSS, 8

$\operatorname{FSS}(\mathbb{P}), 8$

HC, 32

$h^{\varphi}(\xi, k, m), 11$

$\boldsymbol{h}_{S T}, 7$

$\boldsymbol{h}_{T}, 7$

$K_{n}^{\mathbf{c}}, 21$

$K_{n i}^{\mathbf{c}}, 21$

$\Lambda, 6$

$\operatorname{dom}(\overrightarrow{\boldsymbol{\pi}}), 29$

$\mathscr{L}$-formula, 38

$\ln (s), 6$

$\mathscr{L} \Sigma_{n}^{1}, \mathscr{L} \Pi_{n}^{1}, \mathscr{L}(\Sigma+\Pi)_{n}^{1}, 38$

sMF, 29

$\overrightarrow{\text { sMF }}, 29$

$\overrightarrow{\mathbf{s M F}} \|_{<m}, 31$

$\overrightarrow{\mathbf{s M F}} \|_{\geq m}, 31$

$\overrightarrow{\mathbf{S M F}} \|_{m}, 31$

$\overrightarrow{\mathbf{s M F}}_{\omega_{1}}, 29$ 


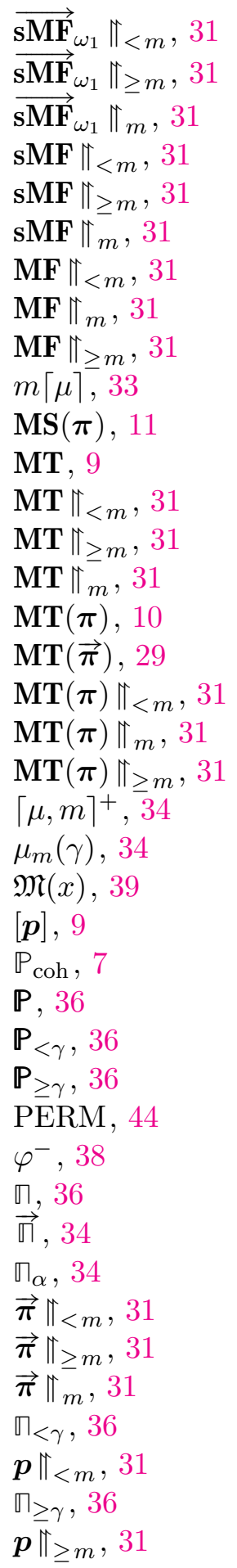

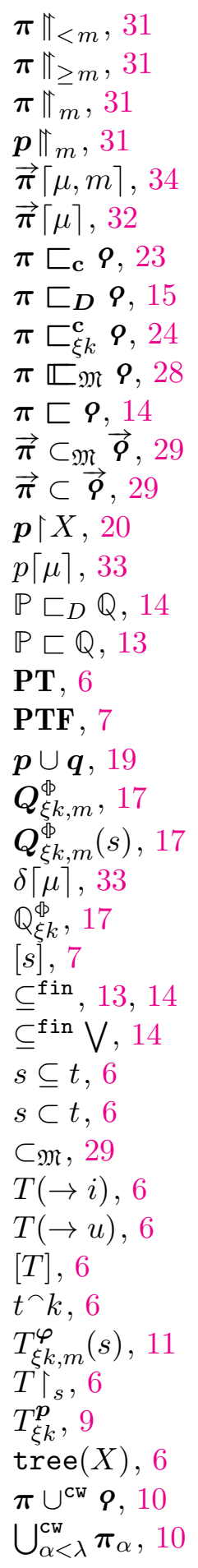


wf orc, 39

$\dot{\boldsymbol{x}}_{\xi k}, 22$

$x_{\xi k}[G], 10$

$\Upsilon_{m}(p), 33$

$z\lceil\mu\rceil, 32$ 